TRANSACTIONS OF THE

AMERICAN MATHEMATICAL SOCIETY

Volume 363, Number 10, October 2011, Pages 5419-5463

S 0002-9947(2011)05323-7

Article electronically published on May 24, 2011

\title{
SOLITON SPHERES
}

\author{
CHRISTOPH BOHLE AND G. PAUL PETERS
}

\begin{abstract}
Soliton spheres are immersed 2-spheres in the conformal 4-sphere $S^{4}=\mathbb{H} \mathbb{P}^{1}$ that allow rational, conformal parametrizations $f: \mathbb{C P}^{1} \rightarrow \mathbb{H} \mathbb{P}^{1}$ obtained via twistor projection and dualization from rational curves in $\mathbb{C P}^{2 n+1}$. Soliton spheres can be characterized as the case of equality in the quaternionic Plücker estimate. A special class of soliton spheres introduced by Taimanov are immersions into $\mathbb{R}^{3}$ with rotationally symmetric Weierstrass potentials that are related to solitons of the mKdV-equation via the ZS-AKNS linear problem. We show that Willmore spheres and Bryant spheres with smooth ends are further examples of soliton spheres. The possible values of the Willmore energy for soliton spheres in the 3-sphere are proven to be $W=4 \pi d$ with $d \in \mathbb{N} \backslash\{0,2,3,5,7\}$. The same quantization was previously known individually for each of the three special classes of soliton spheres mentioned above.
\end{abstract}

\section{INTRODUCTION}

The study of explicitly parametrized surfaces is one of the oldest subjects in differential geometry. For more than two centuries the focus was essentially on local parametrizations. Towards the end of the 20th century, with the rise of the global theory of minimal surfaces [27, 11, 22, 24 and the developments [1, 30, 21, 2] initiated by Wente's solution [36 to the Hopf problem about constant mean curvature surfaces in $\mathbb{R}^{3}$, the focus shifted to rather global considerations. Nevertheless, our knowledge about explicit parametrizations of compact surfaces is still surprisingly rudimentary compared to, for example, the highly developed theory of complex algebraic curves.

An important source of explicitly parametrized spheres and tori is integrable systems theory in combination with complex algebraic geometry. The use of integrable systems methods in the study of general conformal immersions without special curvature properties has been pioneered by Konopelchenko 23. and Taimanov 32, 33, 34. Their approach is based on the Weierstrass representation for conformal immersions into $\mathbb{R}^{3}$ which provides an intimate connection between conformal immersions and Dirac operators; see the survey 35. These ideas were of great influence in the development of quaternionic holomorphic geometry [28, 13] and the starting point of our investigations.

The present paper is devoted to the study of soliton spheres, a class of immersed 2 -spheres in the conformal 4 -sphere $S^{4}$ that admit explicit rational, conformal parametrizations $f: \mathbb{C P}^{1} \rightarrow \mathbb{H} \mathbb{P}^{1}$ obtained by some twistorial construction from

Received by the editors May 20, 2009 and, in revised form, January 8, 2010.

2000 Mathematics Subject Classification. Primary 53C42; Secondary 53A30, 37K25.

Both authors were supported by DFG SPP 1154 "Global Differential Geometry".

(C)2011 American Mathematical Society

Reverts to public domain 28 years from publication 
rational curves in $\mathbb{C P}^{2 n+1}$ and are characterized by the fact that equality holds in the quaternionic Plücker estimate [13]

$$
W \geq 4 \pi[(n+1)(n(1-g)-d)+\mid \text { ord } H \mid],
$$

a fundamental estimate for the Willmore energy $W$ of quaternionic holomorphic line bundles.

The term soliton spheres was introduced by Iskander Taimanov 34 for conformal immersions $f: \mathbb{C P}^{1} \rightarrow \mathbb{R}^{3}=\operatorname{Im} \mathbb{H}$ related to multi-solitons of the modified Korteweg-de Vries $(\mathrm{mKdV})$ equation. With the more general notion of soliton spheres discussed in the present paper, all Willmore spheres and Bryant spheres with smooth ends are examples of soliton spheres.

Section 2 gives an overview of the basic concepts of quaternionic holomorphic geometry. In particular, we explain the Möbius invariant representation of conformally immersed surfaces $f: M \rightarrow S^{4}=\mathbb{H} \mathbb{P}^{1}$ as quotients of quaternionic holomorphic sections. In Section 3 we discuss the relation between equality in the Plücker estimate and twistor holomorphic curves in $\mathbb{H}^{n}$. In Section 4 we show how Taimanov soliton spheres [34] can be treated using the quaternionic language. For this we review the quaternionic version 28 of the Weierstrass representation for conformal immersions into Euclidean 4 -space $\mathbb{R}^{4}=\mathbb{H}$.

In Section [5 we define soliton spheres using the Möbius invariant representation of conformal immersions. We derive an alternative characterization of soliton spheres in terms of the Weierstrass representation which shows that Taimanov soliton spheres are soliton spheres in our sense.

In Sections [6 and 7 we use Darboux and Bäcklund transformations in order to show that Bryant spheres with smooth ends and Willmore spheres are examples of soliton spheres. In Section 8 we prove that all soliton spheres in $\mathbb{R}^{3}$ with Willmore $W \leq 32 \pi$ are Willmore spheres or Bryant spheres with smooth ends and show that the possible Willmore energies of immersed soliton spheres in $\mathbb{R}^{3}$ are $W=4 \pi d$ for $d \in \mathbb{N} \backslash\{0,2,3,5,7\}$. This generalizes previously known results about the quantization of the Willmore energy for Willmore spheres in $\mathbb{R}^{3}$ [9], Taimanov soliton spheres [34], and Bryant spheres with smooth ends [5].

\section{QUATERNionic HOLOMORPHIC GEOMETRY}

The principal idea of quaternionic holomorphic geometry [28, 13, 10] is to approach conformal surface theory using the concept of quaternionic holomorphic line bundles. From this perspective the theory of conformal immersions appears as a "deformation" of the theory of holomorphic curves in $\mathbb{C P}^{n}$. This section gives a quick overview of the basic notions of quaternionic holomorphic geometry. Some special topics of surface theory in the conformal ${ }^{4}$-sphere $S^{4}=\mathbb{H}^{1}$ are discussed in the appendices.

2.1. Quaternionic vector spaces. The quaternions are the 4-dimensional real associative algebra $\mathbb{H}=\mathbb{R} \oplus \mathbb{R} \mathbf{i} \oplus \mathbb{R} \mathbf{j} \oplus \mathbb{R} \mathbf{k}$ with multiplication rules $\mathbf{i}^{2}=\mathbf{j}^{2}=\mathbf{k}^{2}=$ $\mathbf{i j k}=-1$. A quaternion $\lambda=a+b \mathbf{i}+c \mathbf{j}+d \mathbf{k}$ is the sum of its real part $\operatorname{Re}(\lambda)=a$ and its imaginary part $\operatorname{Im}(\lambda)=b \mathbf{i}+c \mathbf{j}+d \mathbf{k}$. We write $\bar{\lambda}=a-b \mathbf{i}-c \mathbf{j}-d \mathbf{k}$ for the quaternionic conjugate. We identify $\mathbb{R}^{4}$ with $\mathbb{H}$ and identify $\mathbb{R}^{3}$ with the space of imaginary quaternions $\operatorname{Im} \mathbb{H}=\mathbb{R} \mathbf{i} \oplus \mathbb{R} \mathbf{j} \oplus \mathbb{R} \mathbf{k}$. 
All quaternionic vector spaces in this paper are right vector spaces. The dual $V^{*}$ of a quaternionic right vector space $V$, naturally a left vector space, is made into a right vector space by defining $\alpha \lambda:=(x \mapsto \bar{\lambda} \alpha(x))$ for $\lambda \in \mathbb{H}$ and $\alpha \in V^{*}$.

2.2. Quaternionic holomorphic line bundles. Let $L$ be a quaternionic line bundle over a Riemann surface $M$, i.e., a vector bundle whose fibers are modeled on $\mathbb{H}$. A complex structure on $L$, that is, a field $J \in \Gamma($ End $L)$ of quaternionic linear endomorphisms with $J^{2}=-\mathrm{Id}$, makes $L$ into a so-called complex quaternionic line bundle. Denote by $*$ the action on 1 -forms of the complex structure of $T M$ and by $K L$ and $\bar{K} L$ the complex quaternionic line bundles whose sections are $L$-valued 1 -forms $\omega$ that satisfy $* \omega=J \omega$ or $* \omega=-J \omega$, respectively.

A quaternionic holomorphic line bundle over a Riemann surface $M$ is a complex quaternionic line bundle $L$ together with a quaternionic holomorphic structure $D$, a differential operator $D: \Gamma(L) \rightarrow \Gamma(\bar{K} L)$ satisfying the Leibniz rule

$$
D(\psi \lambda)=(D \psi) \lambda+(\psi d \lambda)^{\prime \prime}
$$

for all $\psi \in \Gamma(L)$ and $\lambda: M \rightarrow \mathbb{H}$, where $\omega^{\prime \prime}:=\frac{1}{2}(\omega+J * \omega)$. For the kernel of $D$ one writes $H^{0}(L)$ and its elements are called holomorphic sections of $L$. The Leibniz rule implies that a nowhere-vanishing holomorphic section uniquely determines a quaternionic holomorphic structure on a complex quaternionic line bundle.

The complex structure of a complex quaternionic line bundle $L$ induces a decomposition

$$
L=\hat{L} \oplus \hat{L} \mathbf{j}, \quad \hat{L}=\{\psi \in L \mid J \psi=\psi \mathbf{i}\}
$$

of $L$ into isomorphic complex line bundles $\hat{L}$ and $\hat{L} \mathbf{j}$. The quaternionic holomorphic structure

$$
D=\bar{\partial}+Q
$$

decomposes into a $J$ commuting part $\bar{\partial}$, which induces isomorphic complex holomorphic structures on $\hat{L}$ and $\hat{L} \mathbf{j}$, and a $J$ anti-commuting part $Q$ called the Hopf field of $D$ which is a 1 -form with values in $\operatorname{End}(L)$ that satisfies $* Q=-J Q=Q J$. In other words, a quaternionic holomorphic line bundle is the direct sum of a complex holomorphic line bundle $\hat{L}$ with itself plus a Hopf field.

In the case that $M$ is compact, one defines the degree of a complex quaternionic line bundle $L$ over $M$ as the degree of the underlying complex line bundle $\hat{L}$ :

$$
\operatorname{deg}(L):=\operatorname{deg}(\hat{L}) .
$$

\subsection{Holomorphic curves in $\mathbb{H}^{n}$.}

The $n$-dimensional quaternionic projective space $\mathbb{H} \mathbb{P}^{n}$ is the space of 1 -dimensional subspaces $[x]=x \mathbb{H}$ of $\mathbb{H}^{n+1}$. Its tangent space at $[x] \in \mathbb{H} \mathbb{P}^{n}$ is $T_{[x]} \mathbb{H} \mathbb{P}^{1}=$ $\operatorname{Hom}\left([x], \mathbb{H}^{n+1} /[x]\right)$.

A smooth map $M \rightarrow \mathbb{H} \mathbb{P}^{n}$ is the same as a smooth line subbundle $L$ of the trivial bundle $\mathbb{H}^{n+1}$ over $M$. In the following we will neither distinguish between $L$ and the corresponding map $M \rightarrow \mathbb{H}^{n}$ nor between the trivial vector bundle and the corresponding vector space. For example, a smooth map into $\mathbb{H} \mathbb{P}^{n}$ is usually denoted by $L \subset \mathbb{H}^{n+1}$. The derivative of the map $L$ is then given by

$$
\delta=\pi \nabla_{\mid L}
$$

where $\pi: \mathbb{H}^{n+1} \rightarrow \mathbb{H}^{n+1} / L$ is the canonical projection and $\nabla$ denotes the trivial connection on $\mathbb{H}^{n+1}$. A line subbundle $L \subset \mathbb{H}^{n+1}$ of the trivial $\mathbb{H}^{n+1}$-bundle over a 
Riemann surface $M$ is called a holomorphic curve if $L$ admits a complex structure $J \in \Gamma(\operatorname{End}(L)), J^{2}=-$ Id such that

$$
* \delta=\delta J .
$$

A holomorphic curve $\mathbb{H P}^{n}$ is immersed if $\delta$ is nowhere-vanishing. Otherwise, a non-constant holomorphic curve is called branched; cf. Appendix A.1.

\subsection{The quaternionic projective line $\mathbb{H P}^{1}$ as a model of the conformal} 4-sphere $S^{4}$. The affine chart

$$
\sigma: \mathbb{H} \mathbb{P}^{1} \backslash\left\{\left[\begin{array}{l}
1 \\
0
\end{array}\right]\right\} \rightarrow \mathbb{H}, \quad\left[\begin{array}{l}
\lambda \\
1
\end{array}\right] \mapsto \lambda
$$

identifies the quaternionic projective line $\mathbb{H P}^{1}$ with the conformal 4-sphere, i.e., the conformal compactification $S^{4}=\mathbb{H} \cup\{\infty\}$ of $\mathbb{R}^{4}=\mathbb{H}$. Under this identification, the group of projective transformations of $\mathbb{H}^{1}$ corresponds to the orientationpreserving Möbius transformations of $S^{4}$.

An immersed curve $L \subset \mathbb{H}^{2}$ in $\mathbb{H}^{1}$ is holomorphic if and only if the corresponding immersion $M \rightarrow S^{4}$ is conformal: let $L \subset \mathbb{H}^{2}$ be given by

$$
L=\psi \mathbb{H} \subset \mathbb{H}^{2} \quad \text { with } \quad \psi=\left(\begin{array}{l}
f \\
1
\end{array}\right) \in \Gamma(L)
$$

where $f:=\sigma \circ L: M \rightarrow \mathbb{H}$ is $L$ seen in the affine chart $\sigma$. By definition $L$ is a holomorphic curve if and only if $* \delta=\delta J$ for some $J \in \Gamma(L), J^{2}=-$ Id. But this is equivalent to

$$
* d f=-d f R
$$

with smooth $R: M \rightarrow S^{2} \subset \operatorname{Im} \mathbb{H}$, which is then related to $J$ via $J \psi=-\psi R$. In the case that $L$ is immersed, its holomorphicity is thus equivalent to the conformality of the corresponding immersion $f: M \rightarrow \mathbb{H}$, because an immersion $f$ is conformal if and only if there is $R: M \rightarrow \mathbb{H}$ such that $* d f=-d f R$; see [10, Section 2.2].

2.5. Holomorphic curves and linear systems. The so-called Kodaira correspondence is a fundamental relation between holomorphic line bundles and holomorphic curves: let $L \subset \mathbb{H}^{n+1}$ be a holomorphic curve in $\mathbb{H}^{n}$. To avoid technicalities suppose that $L$ is full, i.e., not contained in a linear subspace. The complex structure of $L$ induces a complex structure on the dual bundle

$$
L^{-1} \cong\left(\mathbb{H}^{n+1}\right)^{*} / L^{\perp}
$$

which we again denote by $J$. Let $\pi:\left(\mathbb{H}^{n+1}\right)^{*} \rightarrow\left(\mathbb{H}^{n+1}\right)^{*} / L^{\perp}$ be the canonical projection. Then $L^{-1}$ carries a unique holomorphic structure $D$ such that

$$
D(\pi \psi)=\frac{1}{2}(\pi \nabla \psi+J * \pi \nabla \psi)
$$

for all $\psi \in \Gamma\left(\left(\mathbb{H}^{n+1}\right)^{*}\right)$; cf. [13, Theorem 2.3]. The isomorphism type of the holomorphic line bundle $L^{-1}$ is a projective invariant of the holomorphic curve $L \subset \mathbb{H}^{n+1}$ in $\mathbb{H}^{n}$, which we call the canonical holomorphic line bundle of the curve $L$. For an immersed holomorphic curve $L \subset \mathbb{H}^{2}$ in $\mathbb{H} \mathbb{P}^{1}$ we therefore refer to $L^{-1}$ as one of the Möbius invariant holomorphic line bundles of $L$; see also Section 5.1 ,

As in the complex case, the degree of a holomorphic curve $L \subset \mathbb{H}^{n+1}$ in $\mathbb{H}^{n}$ is defined as the degree of the corresponding quaternionic holomorphic line bundle $L^{-1}$. In other words, the degree of $L \subset \mathbb{H}^{n+1}$ seen as a holomorphic curve is minus the degree of $L$ seen as a complex quaternionic line bundle. 
The holomorphic structure on $L^{-1}$ is the unique holomorphic structure with the property that all projections of constant sections of $\left(\mathbb{H}^{n+1}\right)^{*}$ are holomorphic. The linear system of holomorphic sections of $L^{-1}$ obtained by projection from constant sections of $\left(\mathbb{H}^{n+1}\right)^{*}$ is called the canonical linear system of the curve $L$ and we denote it by $\left(\mathbb{H}^{n+1}\right)^{*} \subset H^{0}\left(L^{-1}\right)$. The canonical linear system of a holomorphic curve is always base point free; i.e., there are no simultaneous zeros of all holomorphic sections in $\left(\mathbb{H}^{n+1}\right)^{*}$.

Let conversely $H \subset H^{0}(\tilde{L})$ be a base point free linear system of a quaternionic holomorphic line bundle $\tilde{L}$. For $p \in M$ denote by $L_{p} \subset H^{*}$ the 1 -dimensional subspace perpendicular to the hyperplane in $H$ of sections vanishing at $p$. Then $L$ is a holomorphic curve, the quaternionic holomorphic line bundle $\tilde{L}$ is isomorphic to $L^{-1}$, and $H$ corresponds to the canonical linear system of $L$; cf. [13, Section 2.6].

In the case of holomorphic curves in $\mathbb{H}^{1}$, Kodaira correspondence can be seen as a representation of conformal immersions as quotients of holomorphic sections: let $L \subset \mathbb{H}^{2}$ be the holomorphic curve given by $L=\left(\begin{array}{c}f \\ 1\end{array}\right) \mathbb{H}$; see Section 2.4. Then the holomorphic sections $e_{1}^{*}$ and $e_{2}^{*}$ of $L^{-1}$ obtained by projecting the standard basis of $\left(\mathbb{H}^{2}\right)^{*}$ are related by

$$
e_{1}^{*}=e_{2}^{*} \bar{f}
$$

Changing the basis of the canonical linear system amounts to a quaternionic fractional linear transformation of $f$, i.e., an orientation-preserving Möbius transformation.

2.6. Weierstrass gaps and flag, dual curve. Let $H \subset H^{0}(L)$ be an $(n+1)-$ dimensional linear system of holomorphic sections of a holomorphic line bundle $L$. The Weierstrass gap sequence of $H$ at a point $p \in M$ is the sequence $0 \leq n_{0}(p)<$ $n_{1}(p)<\ldots<n_{n}(p)$ of possible vanishing orders $\operatorname{ord}_{p}(\psi)$ at $p$ of sections $\psi \in H$. The Weierstrass points, i.e., the points at which the Weierstrass sequence differs from $0,1, \ldots, n$, are isolated; see [13, Section 4.1]. The integer

$$
\operatorname{ord}_{p}(H)=\sum_{k=0}^{n}\left(n_{k}(p)-k\right)
$$

is the Weierstrass order of $H$ at $p$ and $\operatorname{ord}(H)$ is the Weierstrass divisor of $H$. If $M$ is compact the Weierstrass degree $|\operatorname{ord} H|=\sum_{p \in M} \operatorname{ord}_{p}(H)$ is finite.

The members $H_{k \mid p}=\left\{\psi \in H \mid \operatorname{ord}_{p}(\psi) \geq n_{n-k}(p)\right\}$ of the Weierstrass flag of $H$ form continuous subbundles of $H$ of rank $k$ which are smooth away from the Weierstrass points [13. Lemma 4.10]. The line subbundle $H_{0}$ is by definition the dual curve $L^{d}$ of $H$. One can show that, away from the Weierstrass points of $H$, the dual curve $L^{d}$ is a holomorphic curve [13, Theorem 4.2].

2.7. Willmore energy. For compact surfaces, in addition to the invariants such as degree and branching order of the osculating curves known from complex curve theory, quaternionic holomorphic curves have a further invariant: the Willmore energy. The Willmore energy of the holomorphic line bundle $L$ is

$$
W(L)=\frac{1}{2} \int_{M} \operatorname{tr}_{\mathbb{R}}(Q \wedge * Q),
$$


where $\operatorname{tr}_{\mathbb{R}}$ denotes real trace. The 2 -form $\operatorname{tr}_{\mathbb{R}}(Q \wedge * Q)$ is positive and $W(L)=0$ is equivalent to $Q \equiv 0$. In other words, the Willmore energy $W(L)$ of a quaternionic holomorphic line bundle $L$ measures the deviation from the (double of the) underlying complex holomorphic line bundle $\hat{L}$ (Section 2.2).

The Willmore energy of the Möbius invariant holomorphic line bundle $L^{-1}$ of a holomorphic curve $L \subset \mathbb{H}^{2}$ satisfies [10, Section 6.2]

$$
W\left(L^{-1}\right)=\int_{M}\left(|\mathcal{H}|^{2}-G+K^{\perp}\right) d \sigma,
$$

where $\mathcal{H}$ is the mean curvature, $G$ the Gaussian curvature, and $K^{\perp}$ the curvature of the normal bundle of $L$ with respect to any compatible metric on the conformal 4-sphere $S^{4} \cong \mathbb{H P}^{1}$.

\section{Equality in the Plǘcker estimate}

In this section we describe the relation between equality in the Plücker estimate and twistor holomorphic curves.

\subsection{Plücker estimate. The quaternionic Plücker estimate [13, Cor. 4.8]}

$$
W(L) \geq 4 \pi[(n+1)(n(1-g)-d)+\mid \text { ord } H \mid]
$$

gives a lower bound for the Willmore energy $W(L)$ of a quaternionic holomorphic line bundle $L$ of degree $d$ over a compact Riemann surface of genus $g$ with $(n+1)-$ dimensional linear system $H \subset H^{0}(L)$.

3.2. Twistor holomorphic curves. The twistor projection is the map

$$
\mathbb{C P}^{2 n+1} \rightarrow \mathbb{H}^{n}, \quad v \mathbb{C} \mapsto v \mathbb{H},
$$

where $v \mathbb{C}$ denotes the complex line spanned by $v \in \mathbb{H}^{n+1} \backslash\{0\}$ in the complex vector space $\left(\mathbb{H}^{n+1}, \mathbf{i}\right) \cong \mathbb{C}^{2 n+2}$ obtained by restricting the scalar field of the quaternionic right vector space $\mathbb{H}^{n+1}$ to $\mathbb{C}=\mathbb{R} \oplus \mathbb{R} \mathbf{i}$.

Definition. The twistor lift of a holomorphic curve $L \subset \mathbb{H}^{n+1}$ in $\mathbb{H}^{n}$ is the complex line subbundle $\hat{L}=\{\psi \in L \mid J \psi=\psi \mathbf{i}\} \subset\left(\mathbb{H}^{n+1}, \mathbf{i}\right)$. A holomorphic curve in $\mathbb{H P}^{n}$ is called twistor holomorphic if it is the twistor projection of a complex holomorphic curve in $\mathbb{C P}^{2 n+1}$.

Lemma 3.3 ([14]). A holomorphic curve $L \subset \mathbb{H}^{n+1}$ in $\mathbb{H}^{n}$ is twistor holomorphic if and only if its twistor lift $\hat{L}$ is complex holomorphic.

Proof. Let $L \subset \mathbb{H}^{n+1}$ be a holomorphic curve and $E \subset\left(\mathbb{H}^{n+1}\right.$,i $)$ a complex holomorphic line subbundle that twistor projects to $L$. Holomorphicity of $E$ means that every smooth section $\psi \in \Gamma(E)$ satisfies $* \nabla \psi \equiv \nabla \psi \mathbf{i} \bmod E$. But then $* \delta=\delta J$, cf. Section 2.3. yields $\delta \psi \mathbf{i}=* \delta \psi=\delta J \psi$ such that $J \psi=\psi \mathbf{i}$ for all $\psi \in \Gamma(E)$. This implies that $E$ is the twistor lift $\hat{L}$ of $L$.

3.4. Equality in the Plücker estimate. The link between equality in the Plücker estimate, Section 3.1, and twistor holomorphic curves is established by the quaternionic Plücker formula [13, Theorem 4.7]. Let $L$ be a quaternionic holomorphic line bundle of degree $d$ over a compact Riemann surface of genus $g$ and $H \subset H^{0}(L)$ an 
$(n+1)$-dimensional linear system. Then

$$
W(L)-W\left(\left(L^{d}\right)^{-1}\right)=4 \pi[(n+1)(n(1-g)-d)+|\operatorname{ord} H|],
$$

where $L^{d} \subset H$ denotes the dual curve of $H$; see Section 2.6. In particular, although the dual curve $L^{d}$ is only defined away from the Weierstrass points of $H$, the Willmore energy $W\left(\left(L^{d}\right)^{-1}\right)$ is finite.

Equality in the Plücker estimate is thus equivalent to $W\left(\left(L^{d}\right)^{-1}\right)=0$ which, by Lemma 3.8 below, is equivalent to holomorphicity of the twistor lift of $L^{d}$. As we will see in Lemma 3.10, the twistor lift of $L^{d}$ then extends continuously through the Weierstrass points. This yields the following theorem (cf. [13, Section 4.4]).

Theorem 3.5. A linear system of a quaternionic holomorphic line bundle over a compact Riemann surface has equality in the Plücker estimate if and only if its dual curve is twistor holomorphic. The twistor lift then extends holomorphically through the Weierstrass points of the linear system.

3.6. Example: degree formula. Applying the quaternionic Plücker formula to a 1-dimensional linear system yields the so-called degree formula. The degree of a complex holomorphic line bundle over a compact Riemann surface equals the degree of the vanishing divisor of an arbitrary holomorphic section. In the quaternionic case the degree formula additionally involves the Willmore energy: let $L$ be a quaternionic holomorphic line bundle over a compact Riemann surface and $\varphi$ a holomorphic section of $L$. Denote by $H$ the 1-dimensional linear system spanned by $\varphi$. The quaternionic holomorphic line bundle $\left(L^{d}\right)^{-1}$ of the dual curve $L^{d}$ of $H$ (Section 2.6) is isomorphic to $L^{-1}$ restricted to $M \backslash\{$ zeros of $\varphi\}$ equipped with the holomorphic structure $\nabla^{\prime \prime}=\frac{1}{2}(\nabla+J * \nabla)$, where $\nabla$ denotes the connection on $L$ defined by $\nabla \varphi=0$. The Plücker formula applied to $H$ thus becomes

$$
W(L)+4 \pi \operatorname{deg}(L)=W\left(L^{-1}, \nabla^{\prime \prime}\right)+4 \pi|\operatorname{ord} \varphi|,
$$

where $|\operatorname{ord} \varphi|$ is the zero divisor of $\varphi$.

3.7. The canonical complex structure. The canonical complex structure 13 , of an $(n+1)$-dimensional linear system $H$ of holomorphic sections of a quaternionic holomorphic line bundle $L$ without Weierstrass points is the unique complex structure $S \in \Gamma(\operatorname{End}(H)), S^{2}=-1$ that respects the Weierstrass flag (Section 2.6), induces the given complex structure on $L \cong H / H_{n-1}$ and satisfies $H_{n-1} \subset \operatorname{ker}(Q)$ and $\operatorname{im}(A) \subset H_{0}=L^{d}$, where $Q=\frac{1}{4}(S \nabla S-* \nabla S)$ and $A=\frac{1}{4}(S \nabla S+* \nabla S)$ are the so called Hopf fields of $S$. It follows that $S$ restricted to $L^{d}$ is the complex structure of the dual curve. Moreover, the restriction of $Q$ to $L \cong H / H_{n-1}$ coincides with the Hopf field of the holomorphic structure of $L$; cf. Section 2.5 .

The canonical complex structure $S_{L}$ of a full holomorphic curve $L \subset \mathbb{H}^{n+1}$ in $\mathbb{H} \mathbb{P}^{n}$ is defined away from Weierstrass points as the adjoint $S_{L}:=S^{*}$ of the canonical complex structure $S$ of the canonical linear system $\left(\mathbb{H}^{n+1}\right)^{*} \subset H^{0}\left(L^{-1}\right)$; see Section 2.5. Its Hopf fields satisfy $Q_{L}=-A^{*}$ and $A_{L}=-Q^{*}$. In the case of a holomorphic curve in $\mathbb{H P}^{1}$, the canonical complex structure is also called the mean curvature sphere congruence; see Section 7.1 .

Lemma 3.8. Let $L \subset \mathbb{H}^{n+1}$ be a holomorphic curve in $\mathbb{H}^{n}$ and denote by $A$ the Hopf field of the canonical complex structure of $L$. The following properties are 
equivalent:

i) $L$ is twistor holomorphic,

ii) A restricted to $L$ vanishes identically,

iii) A vanishes identically,

iv) the Willmore energy $W\left(L^{-1}\right)$ of the holomorphic line bundle $L^{-1}$ vanishes.

Proof. Let $\psi \in \Gamma(\hat{L})$ be a section of the twistor lift of $L$. Holomorphicity of $L$ implies that $* \nabla \psi=\nabla \psi \mathbf{i}+\psi \alpha$ with $\alpha$ a quaternion-valued 1-form. Using $S \psi=\psi \mathbf{i}$, $\nabla S=2 * Q-2 * A$, and $Q \psi=0$ we obtain

$$
\begin{aligned}
2 * A \psi & =* A \psi+S A \psi=\frac{1}{2}(-(\nabla S) \psi+S(* \nabla S) \psi) \\
& =\frac{1}{2}(-\nabla \psi \mathbf{i}+S \nabla \psi+S * \nabla \psi \mathbf{i}+* \nabla \psi)=\frac{1}{2}(\psi \alpha+S \psi \alpha \mathbf{i}) .
\end{aligned}
$$

Hence $L$ is twistor holomorphic if and only if $A_{\mid L} \equiv 0$, because holomorphicity of the twistor lift $\hat{L}$ of $L$ is equivalent to $\alpha$ being complex-valued.

In order to check that $A_{\mid L} \equiv 0$ if and only if $A \equiv 0$, we prove that $A_{\mid L_{k}} \equiv 0$ implies $A_{\mid L_{k+1}} \equiv 0$, where $L_{k}$ denotes the $k^{t h}$-osculating curve, i.e., the rank $k+1$ subbundles of $\mathbb{H}^{n+1}$ dual to $H_{n-k-1}$ in the Weierstrass flag of the canonical linear system $\left(\mathbb{H}^{n+1}\right)^{*} \subset H^{0}\left(L^{-1}\right)$; see Section 2.6. Let $\psi \in \Gamma\left(L_{k}\right)$ and $A \psi=0$. Then $* A \wedge \nabla \psi=d * A \psi=d * Q \psi=0$, because $d * A=d * Q$ vanishes on $L_{n-1}$. Since $* A=-A S$ and $* \nabla \psi \equiv S \nabla \psi \bmod L_{k}$, this implies $A_{\mid L_{k+1}}=0$.

The last equivalence holds because $-A^{*}$ is the Hopf field of the canonical complex structure of the linear system $\left(\mathbb{H}^{n+1}\right)^{*} \subset H^{0}\left(L^{-1}\right)$ and hence induces the Hopf field of the quaternionic holomorphic line bundle $L^{-1}$.

3.9. Twistor lifts extend continuously through Weierstrass points. In order to complete the proof of Theorem 3.5 it remains to check that the twistor lift of the dual curve of a linear system extends continuously through the Weierstrass points. The proof of this fact given in [13] rests on the false claim that the canonical complex structure (Section 3.7) of a holomorphic curve in $\mathbb{H}^{n}$ extends continuously through the Weierstrass points. A counterexample is the holomorphic curve $L=\psi \mathbb{H}$ in $\mathbb{H} \mathbb{P}^{1}$ defined by $\psi=\left(\begin{array}{c}1+\mathbf{j} z \\ z^{2}\end{array}\right)$ whose canonical complex structure does not extend continuously into $z=0$, because $\psi(0)$ and $\psi^{\prime}(0)$ are linearly dependent over $\mathbb{H}$; cf. Lemma C.2.

We now show how to modify the arguments given in [13] in order to prove that the twistor lift of the dual curve extends continuously into the Weierstrass points.

Lemma 3.10. Let $H \subset H^{0}(L)$ be a linear system of a quaternionic holomorphic line bundle L. The twistor lift of the dual curve $L^{d} \subset H$ of $H$ then extends continuously through the Weierstrass points of $H$.

Proof. Let $S$ be the canonical complex structure of $H$. We need to show that the twistor lift $\widehat{L^{d}}=\left\{\psi \in L^{d} \mid S \psi=\psi \mathbf{i}\right\}$ of $L^{d}$ extends continuously through the Weierstrass points of $H$.

Let $p$ be a Weierstrass point of $H \subset H^{0}(L)$. By Lemma 4.9 in 13 there exists a basis $\psi_{k}, k=0, \ldots, n$ of $H$ that realizes the Weierstrass gap sequence $n_{k}(p)$ of $H$ at $p$ and has the following properties:

(i) There exists an open neighborhood $V$ of $p$ and a smooth map $B: V \rightarrow$ $M(n+1, \mathbb{H})$ that is $\operatorname{GL}(n+1, \mathbb{H})$-valued on $V_{0}:=V \backslash\{p\}$ such that $\psi B^{-1}$ is an adapted frame of the Weierstrass flag $H_{k \mid V_{0}} \subset H$. 
(ii) Let $z: V \rightarrow \mathbb{C}, z(p)=0$ be a coordinate, $Z=\operatorname{diag}\left(1, z^{-1}, \ldots, z^{-n}\right)$, and $W=\operatorname{diag}\left(z^{n_{0}(p)}, \ldots, z^{n_{n}(p)}\right)$. Then there exists $B_{0} \in \mathrm{GL}(n+1, \mathbb{R})$ such that

$$
B=Z\left(B_{0}+O(1)\right) W
$$

where $O(1)$ stands for a continuous map on $V$ that vanishes to first order at $p$.

(iii) The canonical complex structure $S$ satisfies $S \psi B^{-1}=\psi B^{-1} \mathbf{i}$ on $V_{0}$.

Let $\tilde{L}, \tilde{U}: V_{0} \rightarrow \mathrm{GL}(n+1, \mathbb{H})$ be the LU-decomposition of $Z^{-1} B W^{-1}=B_{0}+$ $O(1)$ with diagonal entries of $\tilde{U}$ equal to 1 . The upper triangular matrix $U:=$ $W^{-1} \tilde{U} W$ then converges to the identity matrix when $z \rightarrow 0$. With the lower triangular matrix $L:=Z \tilde{L} W$ one obtains the LU-decomposition $B=L U$ of $B$ restricted to $V_{0}$. On $V_{0}$ the frame

$$
\underline{\psi} B^{-1} L=\underline{\psi} U^{-1}
$$

is adapted to the Weierstrass flag and converges to $\underline{\psi}$ when $z \rightarrow 0$. The section

$$
\varphi:=\underline{\psi} B^{-1} L e_{n+1}=\underline{\psi} U^{-1} e_{n+1}
$$

thus extends to define a continuous section of the trivial bundle $H$ over $V$. It spans the dual curve $L^{d}$ on $V_{0}$ and $\varphi \mathbb{H}$ extends $L^{d}$ continuously into $p$. Because the restriction of $S$ to $L^{d}$ is the complex structure of $L^{d}$ on $V_{0}$ one concludes from (iii) above:

$$
S \varphi=S \underline{\psi} B^{-1} L e_{n+1}=\underline{\psi} B^{-1} \mathbf{i} L e_{n+1}=\varphi \mu
$$

on $V_{0}$, where $\mu=\left(L^{-1} \mathbf{i} L\right)_{(n+1, n+1)}: V_{0} \rightarrow \mathbb{H}$ denotes the lower right entry of the matrix $L^{-1} \mathbf{i} L$. Let $\lambda:=(\tilde{L})_{(n+1, n+1)}$. Then $\lambda(0) \in \mathbb{R} \backslash\{0\}$, because $B_{0}$ is an invertible real matrix, and

$$
\mu=\left(W^{-1} \tilde{L}^{-1} \mathbf{i} \tilde{L} W\right)_{(n+1, n+1)}=z^{-n_{n}(p)} \lambda^{-1} \mathbf{i} \lambda z^{n_{n}(p)} .
$$

Hence $S \varphi=\varphi(\mathbf{i}+O(1))$ and $\varphi(p) \mathbb{C} \subset(\mathbb{H}, \mathbf{i})$ extends the twistor lift $\widehat{L^{d}}$ of the dual curve continuously into the Weierstrass point $p$.

3.11. Two-dimensional linear systems with equality in the Plücker estimate. We have seen, by the counterexample in Section 3.9. that the canonical complex structure of a linear system does not in general extend continuously through the Weierstrass points. However, in the special case of a base point free linear system with equality in the Plücker estimate it does extend smoothly into the Weierstrass points. We prove this only for 2-dimensional linear systems (which is what we need in Section 8.4). The extension to higher-dimensional systems is straightforward, although slightly more involved.

Proposition 3.12. Let $L$ be a quaternionic holomorphic line bundle over a compact Riemann surface $M$ with base point free, 2-dimensional linear system $H \subset H^{0}(L)$ for which equality holds in the Plücker estimate. Then the dual curve $L^{d} \subset H$ of $H$ has a globally defined holomorphic twistor lift and the mean curvature sphere congruence of $L^{d}$ extends smoothly through the branch points of $L^{d}$.

Conversely, let $L^{d} \subset \mathbb{H}^{2}$ be a twistor holomorphic curve whose mean curvature sphere congruence extends smoothly into the branch points. Then $\mathbb{H}^{2}$ induces a base point free, 2-dimensional linear system of holomorphic sections of the quaternionic holomorphic line bundle $L=\mathbb{H}^{2} / L^{d}$ with equality in the quaternionic Plücker estimate. 
Proof. By Theorem 3.5. the curve $L^{d} \subset H$ is twistor holomorphic and its twistor lift $\hat{L}^{d}$ extends holomorphically through the Weierstrass points of $H$. The tangent line congruence $\widehat{L_{1}^{d}}$ of $\widehat{L^{d}}$ is then also globally defined. By Lemma C.2, the canonical complex structure $S$ of $H$ (which, for a 2-dimensional linear system, is the mean curvature sphere congruence $S$ of the dual curve) extends smoothly into a Weierstrass point $p \in M$ of $H$ if and only if $\left(\widehat{L_{1}^{d}} \oplus \widehat{L_{1}^{d}} \mathbf{j}\right)_{\mid p}=H$.

Because $H$ is base point free, the evaluation map ev: $H \rightarrow L, \operatorname{ev}_{p}(\psi)=\psi(p)$ induces a quaternionic bundle isomorphism between $H / L^{d}$ and $L$. The subbundle $\hat{L}=\{\varphi \in L \mid J \varphi=\varphi \mathbf{i}\}$ has a section $\varphi \in \Gamma(\hat{L})$ that does not vanish at $p$. Let $\psi \in \Gamma(H)$ such that $\operatorname{ev}(\psi)=\varphi$. On the open dense set on which $S$ is defined we have $S \psi \equiv \psi \mathbf{i} \bmod L^{d}$, because ev $\circ S=J \circ$ ev by definition of $S$. This implies $\psi \in \Gamma\left(\widehat{L_{1}^{d}}+L^{d}\right)$. Because $\psi(p) \notin L_{\mid p}^{d}$ we obtain that $\widehat{L_{1 \mid p}^{d}} \not \subset L_{\mid p}^{d}$; hence $\widehat{L_{1 \mid p}^{d}}$ is not

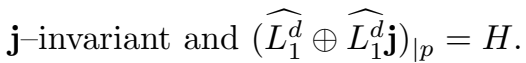

Conversely, the extended mean curvature sphere congruence induces a complex structure on $\left(L^{d}\right)^{\perp} \subset\left(\mathbb{H}^{2}\right)^{*}$ which guarantees that $\left(L^{d}\right)^{\perp}$ is a holomorphic curve. Hence, $L=\mathbb{H}^{2} / L^{d}$ is, as explained in Section 2.5, a quaternionic holomorphic line bundle and the canonical linear system $\mathbb{H}^{2}$ has equality in the Plücker estimate, by Theorem 3.5, because its dual curve $L^{d}$ is twistor holomorphic.

\section{TAIMANOV SOLITON SPHERES}

The term soliton sphere was introduced by Iskander Taimanov 34 for immersions into $\mathbb{R}^{3}$ with rotationally symmetric Weierstrass potentials corresponding to mKdV-solitons. In this section we treat Taimanov soliton spheres in the framework of quaternionic holomorphic geometry using Pedit and Pinkall's Weierstrass representation [28] for conformal immersions into $\mathbb{R}^{4}=\mathbb{H}$.

4.1. Weierstrass representation for conformal immersions into $\mathbb{R}^{4}$. The approach of Taimanov 34 to soliton spheres is based on the generalization of the Weierstrass representation for minimal surfaces to arbitrary conformal immersion into $\mathbb{R}^{3}$. This representation describes the differential of the immersion as the "square" of a Dirac spinor. In contrast to the Kodaira correspondence (Section 2.5), the Weierstrass representation of conformal immersions is not Möbius invariant, but only invariant under similarity transformations.

The quaternionic version of the Weierstrass representation for conformal immersions into $\mathbb{R}^{4}=\mathbb{H}$ is based on the following observation: given a quaternionic holomorphic line bundle $L$, the complex quaternionic line bundle $K L^{-1}$ admits a unique paired holomorphic structure [28, Theorem 4.2] such that

$$
d(\alpha, \psi)=0
$$

for all local holomorphic sections $\alpha \in H^{0}\left(K L_{\mid U}^{-1}\right), \psi \in H^{0}\left(L_{\mid U}\right)$, where $(\alpha, \psi)$ denotes the evaluation pairing $\alpha(\psi)$. This pairing in particular satisfies

$$
*(\alpha, \psi)=(J \alpha, \psi)=(\alpha, J \psi) .
$$

The Weierstrass representation of a conformal immersion $f: M \rightarrow \mathbb{R}^{4}=\mathbb{H}$ into Euclidean 4 -space then reads as follows:

Theorem 4.2 ([28, Theorem 4.3]). Let $f: M \rightarrow \mathbb{R}^{4}=\mathbb{H}$ be a conformal immersion. Then there exist, uniquely up to isomorphism, paired quaternionic holomorphic line 
bundles $L$ and $K L^{-1}$ and nowhere-vanishing holomorphic sections $\psi \in H^{0}(L)$, $\alpha \in H^{0}\left(K L^{-1}\right)$ such that

$$
d f=(\alpha, \psi) .
$$

We call $L$ and $K L^{-1}$ the Euclidean holomorphic line bundles of the conformal immersion $f: M \rightarrow \mathbb{R}^{4}=\mathbb{H}$. By [10, Proposition 8] we have

$$
Q \psi=\psi \frac{1}{2} \overline{\mathcal{H}} d f
$$

and the Willmore energies (Section 2.7) of $L$ and $K L^{-1}$ satisfy

$$
W(L)=W\left(K L^{-1}\right)=\int_{M}|\mathcal{H}|^{2} d \sigma
$$

where $\mathcal{H}$ denotes the mean curvature vector and $d \sigma$ the area element of $f$.

4.3. Weierstrass representation for conformal immersions into $\mathbb{R}^{3}$. The image of the differential $d f$ of a conformal immersion lies in $\operatorname{Im}(\mathbb{H})=\mathbb{R}^{3}$ if and only if

$$
* d f=N d f=-d f N
$$

where $*$ denotes the complex structure on $T^{*} M$ and $N: M \rightarrow S^{2} \subset \operatorname{Im} \mathbb{H}$ is the Gauss map of $f$. This implies that $d f=(\alpha, \psi)$ is $\operatorname{Im}(\mathbb{H})$-valued if and only if $\alpha \mapsto \psi$ induces a holomorphic bundle isomorphism $K L^{-1} \cong L$. A quaternionic holomorphic line bundle $L$ that is isomorphic to $K L^{-1}$ is called a quaternionic spin bundle [28].

Theorem 4.4 ([28, Theorem 4.4]). For a conformal immersion $f: M \rightarrow \operatorname{Im} \mathbb{H}$ there exists, uniquely up to isomorphism, a quaternionic spin bundle with a holomorphic section $\psi$ satisfying

$$
d f=(\psi, \psi) .
$$

Example 4.5 (Minimal surfaces). Let $f: M \rightarrow \mathbb{R}^{3}=\operatorname{Im} \mathbb{H}$ be a conformal immersion. Denote by $L$ the corresponding spin bundle. Then $f$ is minimal if and only if $L$ has vanishing Willmore energy $W(L)=0$. The holomorphic section $\psi \in H^{0}(L)$ in its Weierstrass representation $d f=(\psi, \psi)$ is then the sum $\psi=\psi_{1}+\psi_{2} \mathbf{j}$ of two complex holomorphic spinors $\psi_{1}, \psi_{2} \in \Gamma(\hat{L})$, where $\hat{L}=\{\psi \in L \mid J \psi=\psi \mathbf{i}\}$. The differential of the minimal immersion $f$ is given by

$$
d f=(\psi, \psi)=2 \operatorname{Im}\left(\psi_{1} \otimes \psi_{2}\right)+\mathbf{j}\left(\psi_{1} \otimes \psi_{1}+\overline{\psi_{2} \otimes \psi_{2}}\right),
$$

where $\psi_{i} \otimes \psi_{j}$ stands for the complex-valued $(1,0)-$ form $\left(\psi_{i} \mathbf{j}, \psi_{j}\right)$. This is the wellknown spinor Weierstrass representation of minimal surfaces.

4.6. Weierstrass representation in coordinates. The underlying complex holomorphic line bundle $\hat{L}=\{\psi \in L \mid J \psi=\psi \mathbf{i}\}$ is a complex holomorphic spin bundle over $M$, because $\hat{L} \otimes \hat{L} \rightarrow K, \varphi \otimes \psi \mapsto(\varphi \mathbf{j}, \psi)$ is a bundle isomorphism. In particular, for $z: M \supset U \rightarrow \mathbb{C}$ a local chart with $U$ simply connected, there exists a complex holomorphic section $\varphi$ of $\hat{L}_{\mid U}$ such that $d z=\mathbf{j}(\varphi, \varphi)$. The formula for $Q \psi$ given in Section 4.1 implies

$$
Q \varphi=\varphi \mathbf{k} q d z, \quad q=\frac{H|d f|}{2|d z|} .
$$

The Willmore energy then takes the form $W(L)=2 \mathbf{i} \int q^{2}(z) d z \wedge d \bar{z}$. Writing a quaternionic holomorphic section $\psi \in \mathbb{H}^{0}(L)$ with respect to the complex frame 
$\varphi, \varphi \mathbf{k}$ as $\psi=\varphi \mu_{1}+\varphi \mathbf{k} \mu_{2}$ with $\mu_{1}, \mu_{2}: U \rightarrow \mathbb{C}^{2}$, the holomorphicity $D \psi=0$ of $\psi$ becomes equivalent to $\mu=\left(\begin{array}{c}\mu_{1} \\ \mu_{2}\end{array}\right)$ solving the Dirac equation

$$
\mathcal{D} \mu=0, \quad \mathcal{D}=\left(\begin{array}{cc}
q & \partial \\
-\bar{\partial} & q
\end{array}\right) .
$$

4.7. Taimanov soliton spheres. Let $L$ be a quaternionic spin bundle over $M=$ $\mathbb{C P}^{1}$ and $z: \mathbb{C P}^{1} \backslash\{\infty\} \rightarrow \mathbb{C}$ an affine chart. Then $d z$ is a meromorphic section of the canonical bundle $K$ with a second-order pole at $\infty$ and no zeros. The section $\varphi$ above is thus a meromorphic section of $\hat{L}$ without zeros and with a first-order pole at $\infty$. A function $q: \mathbb{C} \rightarrow \mathbb{R}$ is then a coordinate representation of a smooth Hopf field $Q$ of $L$ if and only if $|w|^{-2} q\left(w^{-1}\right)$ is smooth at $w=0$. Similarly, $\mu_{1}$, $\mu_{2}: \mathbb{C} \rightarrow \mathbb{C}$ is the coordinate representation of a globally smooth section of $L$ if and only if $\left|\mu_{1}\right|^{2}+\left|\mu_{2}\right|^{2}=O\left(|z|^{-2}\right)$ as $|z| \rightarrow \infty$.

Suppose that $q$ is rotationally symmetric, i.e., $q(z)=q(|z|)$. Taimanov then proves [34, Lemma 4] that there are $N+1$ integers $0 \leq n_{0}<\ldots<n_{N}$ and a basis $\left\{\psi_{j}\right\}_{0 \leq j \leq N}$ of $H^{0}(L)$ which is "rotationally symmetric" in the sense that

$$
\psi_{j}=\varphi e^{\frac{\mathbf{i}}{2} y}\left(\nu_{j 1}(x)+\mathbf{k} \nu_{j 2}(x)\right) e^{\frac{\mathbf{i}}{2}\left(2 n_{j}+1\right) y}, \quad z=e^{x+\mathbf{i} y},
$$

where $\nu_{j}: \mathbb{R} \rightarrow \mathbb{C}^{2}$ is a rapidly decaying solution of the ZS-AKNS linear problem

$$
\mathcal{L} \nu_{j}=-\frac{1}{2}\left(2 n_{j}+1\right) \nu_{j}, \quad \mathcal{L}=\left(\begin{array}{cc}
-\partial_{x} & 2 U \\
2 U & \partial_{x}
\end{array}\right), \quad U(x)=q\left(e^{x}\right) e^{x} .
$$

The eigenvalues of $\mathcal{L}$ are preserved under the $\mathrm{mKdV}$ hierarchy and the trace formula for $\mathcal{L}$ implies

$$
\frac{1}{4 \pi} W(L)=2 \int_{-\infty}^{\infty} U^{2}(x) d x \geq \sum_{j=0}^{N}\left(2 n_{j}+1\right) .
$$

Equality in this estimate holds if and only if $U(x)=q\left(e^{x}\right) e^{x}$ is a reflectionless potential of $\mathcal{L}$, i.e., a multi-soliton of the mKdV equation, and $\frac{\mathbf{i}}{2}\left(2 n_{j}+1\right)$ are all points in the spectrum of $L$ with positive imaginary part; cf. 34]. On the other hand, equality in this estimate, is equivalent to equality in the Plücker estimate (Section 3.1) for the full linear system $H^{0}(L)$ of holomorphic sections of the spin bundle $L$. This is because

$$
\begin{aligned}
& (N+1)(N(1-g)-\operatorname{deg}(L))+\operatorname{ord}\left(H^{0}(L)\right) \\
& =(N+1)^{2}+\sum_{j=0}^{N}\left(\operatorname{ord}_{0} \psi_{j}-j\right)+\sum_{j=0}^{N}\left(\operatorname{ord}_{\infty} \psi_{j}-j\right)=\sum_{j=0}^{N}\left(2 n_{j}+1\right),
\end{aligned}
$$

since $g=0, \operatorname{deg}(L)=g-1=-1, \operatorname{ord}_{0}\left(\psi_{j}\right)=\operatorname{ord}_{\infty}(\psi)=n_{j}$, and 0 and $\infty$ are the only possible Weierstrass points.

Theorem 4.8 (Taimanov [34]). Let $L$ be a holomorphic spin bundle $L$ over $\mathbb{C P}^{1}$ whose potential $q$ is rotationally symmetric with respect to some affine coordinate $z: \mathbb{C P}^{1} \backslash\{\infty\} \rightarrow \mathbb{C}$. Then equality in the Plücker estimate holds for $H^{0}(L)$ if and only if the Willmore energy satisfies

$$
W(L)=4 \pi \sum_{j=0}^{N}\left(2 n_{j}+1\right),
$$



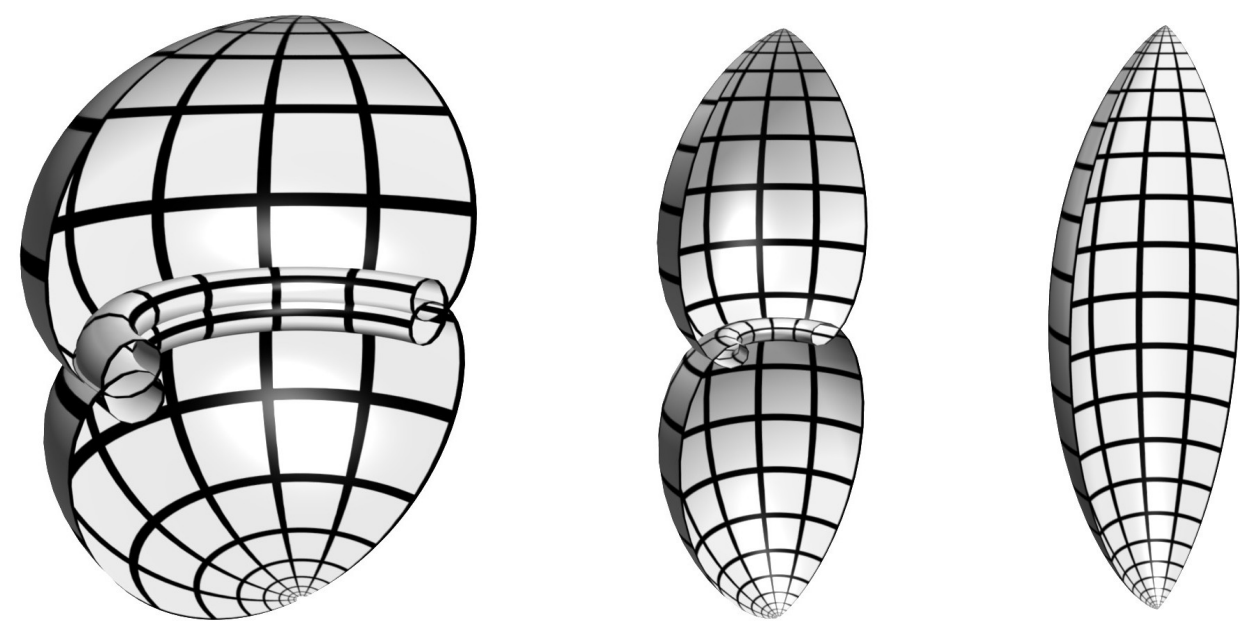

Figure $1 . \quad N=2, n_{0}=0, n_{1}=1, n_{2}=2, \lambda_{0}=2, \lambda_{1}=6, \lambda_{2}=3$.

where $0 \leq n_{0}<\ldots<n_{N}$ are the integers such that there exists a basis of $H^{0}(L)$ consisting of rotationally symmetric holomorphic sections $\left\{\psi_{j}\right\}_{0 \leq j \leq N}$ with $\operatorname{ord}_{0}\left(\psi_{j}\right)=\operatorname{ord}_{\infty}\left(\psi_{j}\right)=n_{j}$.

We call a conformal immersion $f: \mathbb{C P}^{1} \rightarrow \mathbb{R}^{3}$ a Taimanov soliton sphere if its Euclidean holomorphic line bundle has a rotationally symmetric potential with equality in the Plücker estimate for the full linear system. A special example of Taimanov soliton spheres are Dirac spheres 31 which have the most symmetric reflectionless potentials $q(z)=\frac{N+1}{1+|z|^{2}}$. They are soliton spheres with $n_{j}=j, j=$ $0, \ldots, N$ such that $\operatorname{dim} H^{0}(L)=N+1$ and $W(L)=4 \pi(N+1)^{2}$.

Taimanov 34 gives explicit rational formulae for all $q$ and $\psi_{j}$ that may arise in Theorem 4.8. For every $(N+1)$-tuple $0 \leq n_{0}<\ldots<n_{N}$ of integers there is an $\mathbb{R}^{N+1}$-parameter family of $q$ 's and corresponding $\psi_{j}$ 's. Since we are only interested in immersed spheres, we need to start with a base point free linear system and we have to assume that $n_{0}=0$. Because the integers that may be written as the sum of 1 with other pairwise distinct odd integers are $\mathbb{N} \backslash\{0,2,3,5,7\}$ we obtain the following corollary.

Corollary 4.9. The possible Willmore energies of Taimanov soliton spheres are $W \in 4 \pi(\mathbb{N} \backslash\{0,2,3,5,7\})$.

Example 4.10 (Catenoid cousins). Taimanov soliton spheres for $N=1, n_{0}=0$, $n_{1}=\mu, \lambda_{0}=\frac{\mu+1}{\mu}$, and $\lambda_{1}=\frac{(\mu+1)(2 \mu+1)}{\mu}, \mu \in \mathbb{N} \backslash\{0\}$ (cf. 34] for the meaning of $\left.\lambda_{j}\right)$ are the catenoid cousins that have smooth ends; see Example 6.11 for images.

Example 4.11 (Rotationally symmetric soliton spheres). Figures 14 show rotationally symmetric, branched Taimanov soliton spheres, i.e., primitives of $\left(\psi_{j}, \psi_{j}\right)$, $j=0, \ldots, N$. The first example in each figure is immersed; the other examples have branch points of order $2 n_{i}$ on the axis of revolution.

Example 4.12 (More Taimanov soliton spheres). Linear combinations of the $\psi_{j}$ lead to non-rotationally symmetric Taimanov soliton spheres. Figure 5 shows a 

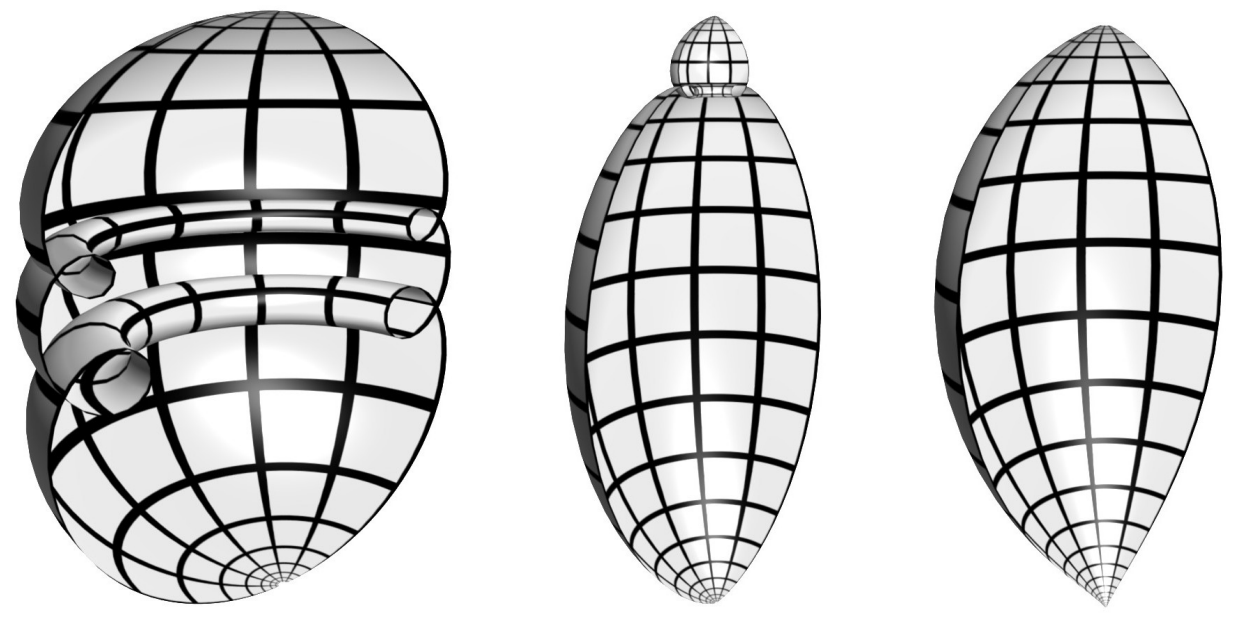

Figure 2. $N=2, n_{0}=0, n_{1}=1, n_{2}=2, \lambda_{0}=2, \lambda_{1}=6, \lambda_{2}=120$.
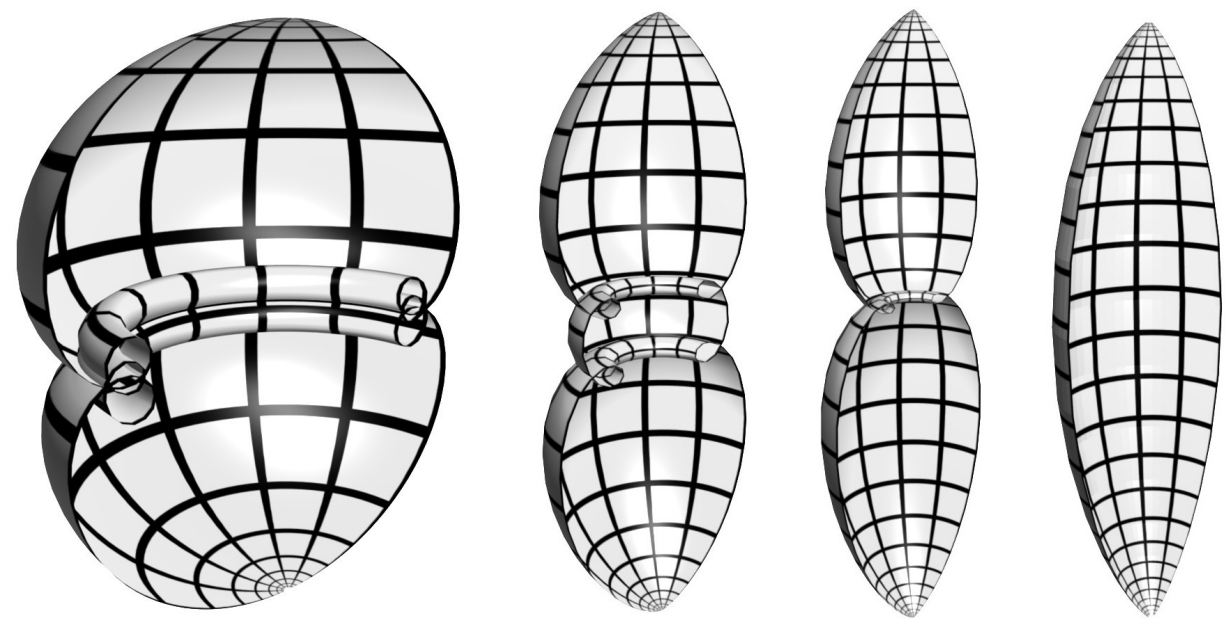

FiguRE 3. $N=3, n_{0}=0, n_{1}=1, n_{2}=2, n_{3}=3, \lambda_{0}=4$, $\lambda_{1}=48, \lambda_{2}=120, \lambda_{3}=120$.

deformation of the first into the third surface in Figure 10 through a family of surfaces $\int(\psi, \psi)$, where $\psi$ is a linear combination of $\psi_{0}$ and $\psi_{2}$. The last surface in the figure is branched; all others are immersed.

\section{SOLITON SPHERES}

We define soliton spheres in terms of the Möbius invariant holomorphic line bundles of a conformal immersion $f: \mathbb{C P}^{1} \rightarrow \mathbb{H}^{1}$. We also give a Euclidean characterization of soliton spheres based on the Weierstrass representation. This in particular implies that Taimanov soliton spheres are examples of soliton spheres in our sense. 

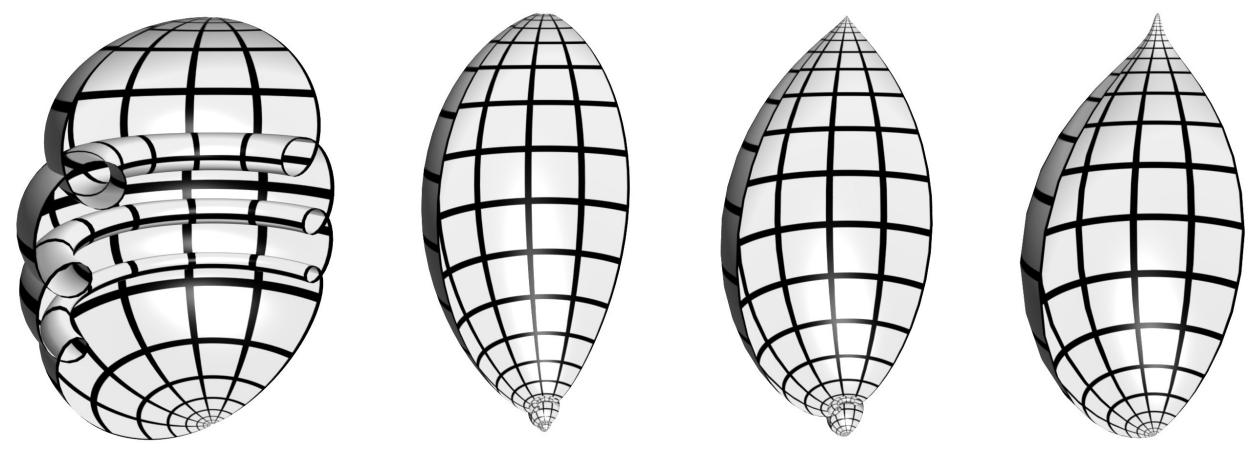

FiguRe 4. $N=3, n_{0}=0, n_{1}=1, n_{2}=2, n_{3}=3, \lambda_{0}=6$, $\lambda_{1}=720, \lambda_{2}=120, \lambda_{3}=1$.
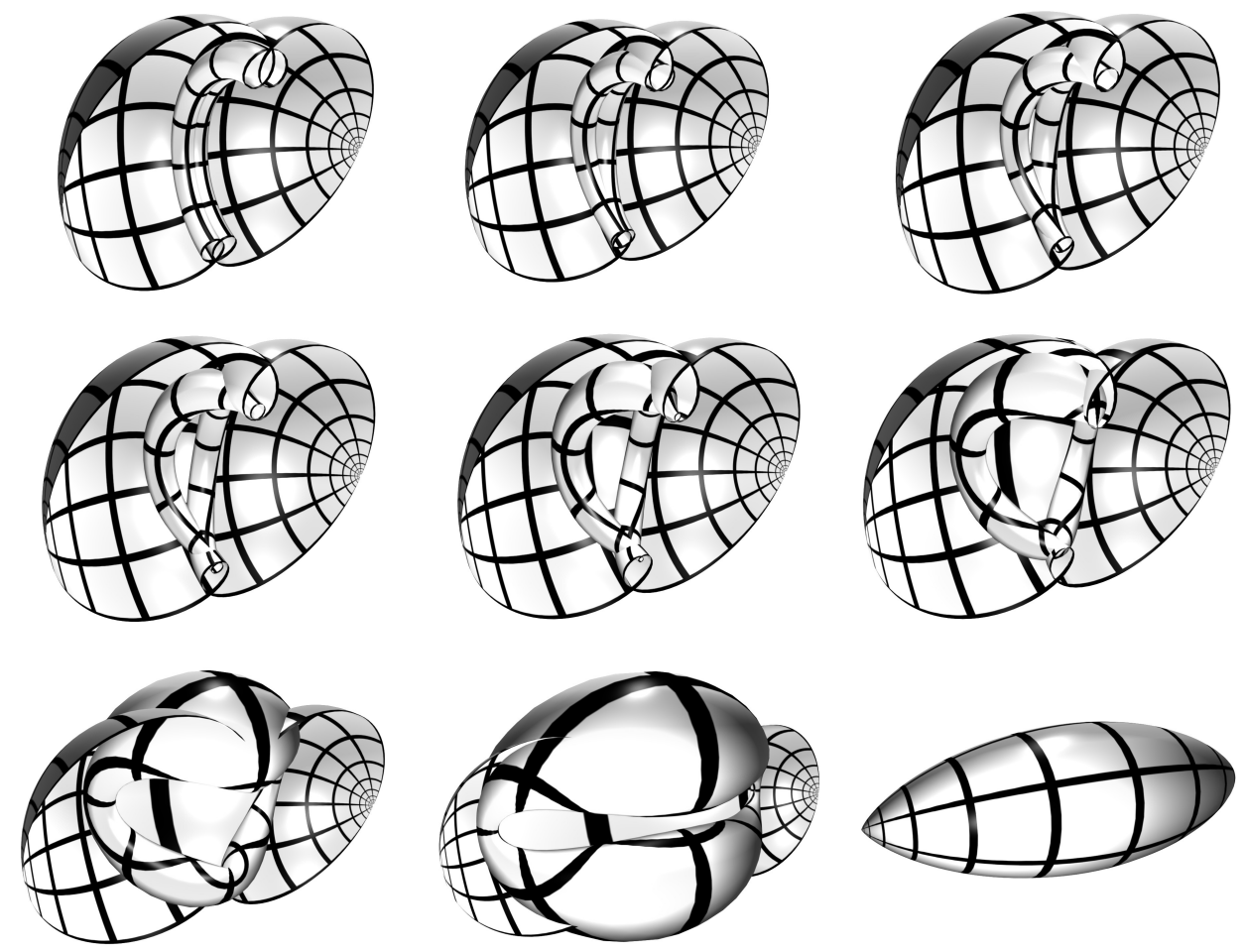

Figure 5. Deformation between the first and third surface in Figure1 through a family of Taimanov soliton spheres with isomorphic Euclidean holomorphic line bundles.

5.1. Soliton spheres. Let $f: \mathbb{C P}^{1} \rightarrow \mathbb{H} \mathbb{P}^{1}$ be a conformal immersion of a Riemann surface into the conformal 4 -sphere. Denote by $L \subset \mathbb{H}^{2}$ the corresponding holomorphic curve and by $L^{\perp} \subset\left(\mathbb{H}^{2}\right)^{*}$ its dual holomorphic curve. In the following, by the Möbius invariant holomorphic line bundles of $L$ we mean the quaternionic line bundles $L^{-1}=\left(\mathbb{H}^{2}\right)^{*} / L^{\perp}$ and $\left(L^{\perp}\right)^{-1}=\mathbb{H}^{2} / L$ with holomorphic structures as defined in Section 2.5. The spaces of constant sections of $\left(\mathbb{H}^{2}\right)^{*}$ and $\mathbb{H}^{2}$ project 
to the canonical linear systems $\left(\mathbb{H}^{2}\right)^{*} \subset H^{0}\left(L^{-1}\right)$ and $\mathbb{H}^{2} \subset H^{0}\left(\mathbb{H}^{2} / L\right)$. Soliton spheres are immersions for which one of the canonical linear systems is contained in a linear system with equality in the Plücker estimate (Section 3.1).

Definition. A conformal immersion $f: \mathbb{C P}^{1} \rightarrow \mathbb{H}^{1}$ into the conformal 4-sphere is called a soliton sphere if one of the two Möbius invariant holomorphic line bundles admits a linear system with equality in the Plücker estimate that contains the canonical linear system.

Two fundamental properties of soliton spheres are immediate consequences of the definition: first, the notion of soliton spheres is Möbius invariant. Second, given a soliton sphere $L \subset \mathbb{H} \mathbb{P}^{1}$, Theorem 3.5 implies that either $L$ or $L^{\perp}$ is the projection to $\mathbb{H} \mathbb{P}^{1}$ of a holomorphic curve in $\mathbb{H} \mathbb{P}^{n}$ that is the dual curve (Section 2.6) of a twistor holomorphic curve in $\mathbb{H}^{n}$ (Section 3.2). In other words, every soliton sphere is obtained from a rational curve in $\mathbb{C P}^{2 n+1}$ via twistor projection $\mathbb{C P}^{2 n+1} \rightarrow \mathbb{H} \mathbb{P}^{n}$, dualization and projection to $\mathbb{H} \mathbb{P}^{1}$ (possibly followed by a dualization in $\mathbb{H} \mathbb{P}^{1}$ ), i.e., by

$$
\mathbb{C P}^{1} \stackrel{\text { rational }}{\longrightarrow} \mathbb{C P}^{2 n+1} \stackrel{\text { twistor }}{\longrightarrow} \mathbb{H} \mathbb{P}^{n} \stackrel{\text { dualization }}{\leftarrow----\rightarrow} \mathbb{H} \mathbb{P}^{n} \stackrel{\text { projection }}{\longrightarrow} \mathbb{H} \mathbb{P}^{1}
$$

or the same sequence followed by a dualization in $\mathbb{H} \mathbb{P}^{1}$, depending on whether $H^{0}\left(L^{-1}\right)$ or $H^{0}\left(\mathbb{H}^{2} / L\right)$ has a linear system with equality. The dual curve of a holomorphic curve $L$ in $\mathbb{H P}^{n}$ is the solution of a system of quaternionic linear equations whose coefficients are the $(n-1)^{\text {th }}$ derivatives of a generic section of $L$. Hence, every soliton sphere admits a rational, conformal parametrization.

Definition. The soliton number of a soliton sphere that is not the round sphere is defined as the minimal number $n$ for which one of the canonical linear systems is contained in an $(n+1)$-dimensional linear system with equality in the Plücker estimate. The soliton number of the round sphere is defined to be 0 .

The soliton number $n$ is the smallest number for which a soliton sphere can be obtained via the above construction from a rational curve in $\mathbb{C P}^{2 n+1}$. The only 0 -soliton sphere is the round sphere. A 1-soliton sphere is either twistor holomorphic or the dual of a twistor holomorphic curve; that is, all 1-soliton spheres are superconformal Willmore spheres (cf. Section 8.2 of [10]) and vice versa. Examples of 2-soliton spheres are Bryant spheres with smooth ends (Section 6) and non-superconformal Willmore spheres (Section [7).

5.2. Characterization in terms of the Weierstrass representation. We now give a Euclidean characterization of soliton spheres in terms of the Weierstrass representation. As an application we show that Taimanov soliton spheres are also soliton spheres as in Section [5.1.

Let $L \subset \mathbb{H}^{2}$ be an immersed holomorphic curve and fix a point $\infty \in \mathbb{H} \mathbb{P}^{1}$ that does not lie in the image of $L$. Without loss of generality we may assume that $\infty=\left[e_{1}\right]$, where $e_{1}, e_{2} \in \mathbb{H}^{2}$ denotes the standard basis. This basis of $\mathbb{H}^{2}$ defines the affine chart (Section 2.4)

$$
\sigma: \mathbb{H} \mathbb{P}^{1} \backslash\{\infty\} \rightarrow \mathbb{H}, \quad\left[\begin{array}{l}
\lambda \\
1
\end{array}\right] \mapsto \lambda .
$$

The affine chart is in fact defined uniquely up to similarity transformations by the choice of $\infty=\left[e_{1}\right] \in \mathbb{H} \mathbb{P}^{1}$. The immersed holomorphic curve $L$ can then be written 
as

$$
L=\psi \mathbb{H} \quad \text { with } \quad \psi=\left(\begin{array}{l}
f \\
1
\end{array}\right),
$$

where $f=\sigma \circ L: M \rightarrow \mathbb{H}$ is the affine representation of $L$, i.e., the conformal immersion into $\mathbb{H}$ corresponding to $L$ via $\sigma$.

Let $e_{1}^{*}, e_{2}^{*} \in \Gamma\left(L^{-1}\right)$ be the projections to the first and second coordinates of $\mathbb{H}^{2}$ seen as sections of $L^{-1}=\left(\mathbb{H}^{2}\right)^{*} / L^{\perp}$. The canonical linear system (Section 2.5) of $L$ is then spanned by the holomorphic sections $e_{1}^{*}$ and $e_{2}^{*}$ whose quotient is $\bar{f}$, i.e.,

$$
e_{1}^{*}=e_{2}^{*} \bar{f} .
$$

Hence $f: \mathbb{C P}^{1} \rightarrow \mathbb{H}$ is a soliton sphere if and only if $f$ or $\bar{f}$ is the quotient of two quaternionic holomorphic sections that are contained in a linear system with equality in the Plücker estimate, because replacing $f$ by $\bar{f}$ is equivalent to replacing $L$ by $L^{\perp}$ and interchanging the Möbius invariant and the Euclidean holomorphic line bundles.

The choice of $\infty \in \mathbb{H} \mathbb{P}^{1}$ defines a unique flat connection $\nabla$ on $L^{-1}$ which satisfies $\nabla e_{2}^{*}=0$ (where $e_{2}^{*}$ is perpendicular to $\infty$ ). The induced connection $\nabla$ on $L$ then satisfies $\nabla \psi=0$. Moreover $d^{\nabla}$ induces a quaternionic holomorphic structure on $K L^{-1}$ (in the same way as $d$ defines the complex holomorphic structure on complexvalued $(1,0)$-forms) and $\nabla^{\prime \prime}=\frac{1}{2}(\nabla+J * \nabla)$ induces a quaternionic holomorphic structure on $L$ with respect to which $\alpha=\nabla e_{1}^{*}=e_{2}^{*} d \bar{f}$ and $\psi$ are holomorphic sections of $K L^{-1}$ and $L$, respectively. The holomorphic structures thus defined make $K L^{-1}$ and $L$ into paired holomorphic line bundles, and

$$
d f=(\alpha, \psi)
$$

is the Weierstrass representation of $f$. Theorem 4.2 implies that the line bundles $L$ and $K L^{-1}$ with holomorphic structures $d^{\nabla}$ and $\nabla^{\prime \prime}$ are the Euclidean holomorphic line bundles of $f$.

Theorem 5.3. A conformal immersion $f: \mathbb{C P}^{1} \rightarrow \mathbb{H}=\mathbb{H} \mathbb{P}^{1} \backslash\{\infty\}$ is a soliton sphere if and only if one of the two holomorphic sections of the Euclidean holomorphic line bundles arising in the Weierstrass representation of $f$ is contained in a linear system with equality in the Plücker estimate.

Corollary 5.4. Immersed Taimanov soliton spheres are soliton spheres.

Proof of Theorem [5.3. The holomorphic structure of the Möbius invariant holomorphic line bundle $L^{-1}=\left(\mathbb{H}^{2}\right)^{*} / L^{\perp}$ is given by $D=\nabla^{\prime \prime}$, where as above $\nabla$ is the connection defined by $\nabla e_{2}^{*}=0$. It is sufficient to show that $L^{-1}$ admits a linear system that contains $e_{1}^{*}$ and $e_{2}^{*}$ and has equality in the Plücker estimate if and only if $K L^{-1}$ admits a linear system with equality that contains $\alpha$. This follows from Lemma 5.5 below, because $\nabla e_{2}^{*}=0, \nabla e_{1}^{*}=\alpha$, and $\mathbb{C P}^{1}$ is simply connected.

Lemma 5.5. Let $L$ be a quaternionic holomorphic line bundle over a compact Riemann surface with a nowhere-vanishing holomorphic section $\varphi_{0}$. Let $\nabla$ be the flat connection on $L$ defined by $\nabla \varphi_{0}=0$ and $d^{\nabla}$ the induced quaternionic holomorphic structure on $K L$.

Then $\nabla$ induces a linear map from $H^{0}(L)$ to $H^{0}(K L)$ which maps every $(n+1)$ dimensional linear system $H \subset H^{0}(L)$ containing $\varphi_{0}$ to an $n$-dimensional linear system $\nabla H \subset H^{0}(K L)$. The linear system $H$ has equality in the Plücker estimate if and only if $\nabla H$ has equality. 
Proof. The Leibniz rule (Section 2.2) and $D=\nabla^{\prime \prime}$ imply that a section $\varphi \in \Gamma(L)$ is holomorphic if and only if $\nabla \varphi \in \Gamma(K L)$. Because $\nabla$ is flat, it thus induces a quaternionic linear map $\varphi \in H^{0}(L) \mapsto \nabla \varphi \in H^{0}(K L)$ with kernel $\varphi_{0} \mathbb{H}$. The Weierstrass gap sequences $\left(n_{k}\right)$ of $H$ and $\left(\tilde{n}_{k}\right)$ of $\nabla H$ are related by $\tilde{n}_{k}=n_{k+1}-1$. Hence $|\operatorname{ord} H|=|\operatorname{ord} \nabla H|$; cf. Section [2.6. Furthermore, $\operatorname{deg}(K L)=\operatorname{deg}(L)+$ $2(g-1)$ and $W(K L)=W(L)+4 \pi \operatorname{deg}(L)$. The latter follows from the degree formula (Section 3.6) applied to $\varphi=\varphi_{0}$ using the fact that $W(K L)=W\left(L^{-1}, \nabla^{\prime \prime}\right)$ (where $\nabla$ denotes the dual of $\nabla$ above), because $\left(K L, d^{\nabla}\right)$ and $\left(L^{-1}, \nabla^{\prime \prime}\right)$ are paired bundles (Section 4.1). Plugging all these identities into the Plücker estimate (Section 3.1) shows that equality for $H$ is equivalent to equality for $\nabla H$.

\section{Darboux transformation AND SOLITON SPHERES: BRYANT SPHERES WITH SMOOTH ENDS}

We characterize 2 -soliton spheres as those conformal immersions of $\mathbb{C P}^{1}$ into $S^{4}$ that admit a non-trivial twistor holomorphic Darboux transform. This generalizes our previous result [5] that Bryant spheres with smooth ends are soliton spheres and allows a new geometric proof of this fact.

6.1. 2-Soliton spheres and Darboux transformations. Let $L \subset \mathbb{H}^{2}$ be an immersed holomorphic curve. The canonical projection $\pi: \mathbb{H}^{2} \rightarrow \mathbb{H}^{2} / L$ onto the Möbius invariant holomorphic line bundle $\mathbb{H}^{2} / L$, cf. Section 5.1, induces a 1-1correspondence

$$
\left\{\psi^{\sharp} \in \Gamma\left(\mathbb{H}^{2}\right) \mid \nabla \psi^{\sharp} \in \Omega^{1}(L)\right\} \longrightarrow H^{0}\left(\mathbb{H}^{2} / L\right), \quad \psi^{\sharp} \longmapsto \pi \psi^{\sharp} .
$$

The formula in Section 2.5 for the holomorphic structure of $\mathbb{H}^{2} / L$ shows that $\pi \psi^{\sharp} \in$ $\Gamma\left(\mathbb{H}^{2}\right)$ is indeed holomorphic if $\nabla \psi^{\sharp} \in \Omega^{1}(L)$. The correspondence is bijective because $\delta=\pi \nabla_{\mid L}: L \rightarrow K\left(\mathbb{H}^{2} / L\right)$ is a bundle isomorphism since $L$ is an immersion. The section $\psi^{\sharp} \in \Gamma\left(\mathbb{H}^{2}\right)$ is called the prolongation of the holomorphic section $\varphi=$ $\pi \psi^{\sharp}$.

A Darboux transform [4] of a conformal immersion $L \subset \mathbb{H}^{2}$ is a map $L^{\sharp} \subset \mathbb{H}^{2}$ defined away from the zeros of the prolongation $\psi^{\sharp}$ of a holomorphic section $\varphi=$ $\pi \psi^{\sharp} \in H^{0}\left(\mathbb{H}^{2} / L\right)$ by the lines $L^{\sharp}=\psi^{\sharp} \mathbb{H} \subset \mathbb{H}^{2}$ spanned by $\psi^{\sharp}$ (in the case of a non-simply connected surface, one has to allow for holomorphic sections with monodromy). Darboux transforms of $L$ thus correspond to 1-dimensional linear systems of holomorphic sections of $\mathbb{H}^{2} / L$. A Darboux transform is constant if and only if the 1-dimensional linear system is contained in the 2-dimensional canonical linear system; otherwise it is a branched conformal immersion.

Theorem 6.2. A conformal immersion of $\mathbb{C P}^{1}$ into $\mathbb{H}^{1}$ is a 2-soliton sphere if and only if it has a non-constant twistor holomorphic Darboux transform.

Proof. The theorem is a direct consequence of the following proposition.

Proposition 6.3. Let $L^{\sharp} \subset \mathbb{H}^{2}$ be a non-constant Darboux transform of a conformally immersed sphere $L \subset \mathbb{H}^{2}$ in $\mathbb{H} \mathbb{P}^{1}$. Then $L^{\sharp}$ is twistor holomorphic if and only if the corresponding 1-dimensional linear system of $\mathbb{H}^{2} / L$ together with its canonical linear system spans a 3-dimensional linear system with equality in the Plücker estimate.

Proof. Let $\infty=e_{1} \mathbb{H}$ be a point that does not lie on the image of the immersed sphere $L \subset \mathbb{H}^{2}$ in $\mathbb{H} \mathbb{P}^{1}$. As in Section 2.4, denote by $f: \mathbb{C P}^{1} \rightarrow \mathbb{H}$ the representation 
of $L$ in the affine chart corresponding to a basis $e_{1}, e_{2} \in \mathbb{H}^{2}$, i.e., $L=\psi \mathbb{H}$ for $\psi=\left(\begin{array}{l}f \\ 1\end{array}\right)$. The canonical linear system of $\mathbb{H}^{2} / L$ is then spanned by the sections $\varphi:=\pi e_{1}$ and $\pi e_{2}=-\varphi f$. By the Leibniz rule in Section 2.2 , a section $\varphi h$ of $\mathbb{H}^{2} / L$ is holomorphic if and only if $* d h=N d h$ with $N: \mathbb{C P}^{1} \rightarrow \mathbb{H}, N^{2}=-1$ defined by $J \varphi=\varphi N$. Hence $\varphi h$ is holomorphic if and only if there exists $g: \mathbb{C P}^{1} \rightarrow \mathbb{H}$ such that

$$
d f g+d h=0
$$

The prolongation $\psi^{\sharp}$ of $\varphi h$ is then given by

$$
\psi^{\sharp}=\left(\begin{array}{l}
f \\
1
\end{array}\right) g+\left(\begin{array}{l}
1 \\
0
\end{array}\right) h .
$$

The Darboux transform $L^{\sharp} \subset \mathbb{H}^{2}$ corresponding to $\varphi h$ is defined away from the zeros of $\psi^{\sharp}$ as the line subbundle spanned by $\psi^{\sharp}$. Its affine part $f^{\sharp}$ is defined away from the zeros of $g$ and satisfies

$$
f^{\sharp}=f+h g^{-1} \quad \text { and } \quad d f^{\sharp}=h d\left(g^{-1}\right) .
$$

This proves the following lemma.

Lemma 6.4. The map $f^{\sharp}=f+h g^{-1}$ is a Darboux transform of the conformal immersion $f$ if and only if $d f g+d h=0$.

Unless $\varphi h$ is contained in the canonical linear system, away from the isolated zeros of $g, h$, and $d g$, the affine part $f^{\sharp}$ of $L^{\sharp}$ is a conformal immersion.

Lemma 6.5. Let $f^{\sharp}$ be a Darboux transform of $f$ given by $f^{\sharp}=f+h g^{-1}$ with $d f g+d h=0$ and nowhere-vanishing $g$, $h$, and $d g$. Then

i) $f^{\sharp}$ is twistor holomorphic if and only if $g$ is twistor holomorphic,

ii) $f^{\sharp}$ is totally umbilic if and only if $g$ is twistor holomorphic and $h^{-1}$ is Euclidean minimal, and

iii) $f^{\sharp}$ is planar if and only if both $g^{-1}$ and $h^{-1}$ are twistor holomorphic and Euclidean minimal.

Proof. From $d f^{\sharp}=h d\left(g^{-1}\right)$ we obtain that the right normal vectors (cf. Appen$\operatorname{dix}$ A.3. of $f^{\sharp}$ and $g^{-1}$ coincide:

$$
R_{f^{\sharp}}=R_{g^{-1}} .
$$

On the other hand $d\left(g^{-1}\right)=h^{-1} d f^{\sharp}$ implies $0=d\left(h^{-1}\right) \wedge d f^{\sharp}$ and thus

$$
N_{f^{\sharp}}=-R_{h^{-1}} \text {. }
$$

The lemma now follows, because (as shown in Appendix A.4) a conformal immersion is twistor holomorphic if and only if its inversion is twistor holomorphic if and only if $d R^{\prime \prime}=0$; it is Euclidean minimal if and only if $d R^{\prime}=0$; it is totally umbilic if and only if $d N^{\prime \prime}=d R^{\prime \prime}=0$; it is planar if and only if its normal vectors $N$ and $R$ are both constant.

Proof of Proposition 6.3 continued. We have to show that $L^{\sharp}=\left(\begin{array}{c}f^{\sharp} \\ 1\end{array}\right) \mathbb{H}$ is twistor holomorphic if and only if the linear system $H$ spanned by $\varphi, \varphi f$, and $\varphi h$ has equality in the Plücker estimate. Applying Lemma 5.5 to the nowhere-vanishing holomorphic section $\varphi$ shows that equality in the Plücker estimate for $H$ is equivalent to equality in the Plücker estimate for the 2 -dimensional linear system $\nabla H$ of $K\left(\mathbb{H}^{2} / L\right)$ spanned by $\varphi d f, \varphi d h=-\varphi d f g$. Theorem 3.5 now implies that equality 
for $\nabla H$ is equivalent to $g$ being twistor holomorphic, since $\left(\begin{array}{l}g \\ 1\end{array}\right) \mathbb{H} \subset \mathbb{H}^{2}$ is the dual curve of $\nabla H$. This proves the claim, because $g$ is twistor holomorphic if and only if $f^{\sharp}$ is twistor holomorphic (Lemma 6.5).

Remark 6.6. Proposition 6.3 holds verbatim for compact Riemann surfaces of higher genus if one allows for linear systems with monodromy.

Remark 6.7. In general, a Darboux transform of a conformal immersion $L \subset \mathbb{H}^{2}$ may not extend smoothly through the isolated zeros of the corresponding holomorphic section of $\mathbb{H}^{2} / L$. We now show that, in the situation of Proposition 6.3, the Darboux transform $L^{\sharp}$ extends smoothly through the zeros of the defining holomorphic section of $\mathbb{H}^{2} / L$ and has a globally smooth twistor lift (which is hence a rational curve in $\left.\mathbb{C P}^{3}\right)$.

Let $L \subset \mathbb{H}^{2}$ be a conformal immersion and $\varphi h$ a holomorphic section of $\mathbb{H}^{2} / L$ that, together with the canonical linear system, spans a 3-dimensional linear system $H \subset H^{0}\left(\mathbb{H}^{2} / L\right)$ with equality in the Plücker estimate. Then $L^{\sharp}=\psi^{\sharp} \mathbb{H}, \psi^{\sharp}=$ $\left(\begin{array}{l}f \\ 1\end{array}\right) g+\left(\begin{array}{l}1 \\ 0\end{array}\right) h$ is defined and smooth away from the common zeros of $g$ and $h$. Moreover, $L^{\sharp}$ is a holomorphic curve with complex structure $J^{\sharp} \psi^{\sharp}=-\psi^{\sharp} R_{g}$ for $R_{g}$ the right normal of $g$, because $\nabla \psi^{\sharp}=\psi d g$.

By Theorem 3.5, the curve $\left(\begin{array}{l}g \\ 1\end{array}\right) \mathbb{H}$ has a globally defined holomorphic twistor lift that locally is of the form $\left(\begin{array}{l}g_{1}+\mathbf{j} g_{2} \\ g_{3}+\mathbf{j} g_{4}\end{array}\right) \mathbb{C} \subset\left(\mathbb{H}^{2}, \mathbf{i}\right)$ with complex holomorphic functions $g_{1}, \ldots, g_{4}$. Let $p$ be a common zero of $g$ and $h$. Without loss of generality we may assume that $g_{3}+\mathbf{j} g_{4}$ does not vanish at $p$, because $g$ has no "poles". Now $R_{g}=-\left(g_{3}+\mathbf{j} g_{4}\right) \mathbf{i}\left(g_{3}+\mathbf{j} g_{4}\right)^{-1}$ implies that the twistor lift of $L^{\sharp}$ is locally given as the complex line spanned by

$$
\psi^{\sharp}\left(g_{3}+\mathbf{j} g_{4}\right)=\left(\begin{array}{l}
f \\
1
\end{array}\right)\left(g_{1}+\mathbf{j} g_{2}\right)+\left(\begin{array}{l}
1 \\
0
\end{array}\right) h\left(g_{3}+\mathbf{j} g_{4}\right) .
$$

If $n$ is the vanishing order of $g$ at $p$, then $h$ vanishes to order $n+1$ at $p$, because $d f g=-d h$. Since $g_{3}+\mathbf{j} g_{4}$ does not vanish, $n$ is the vanishing order of $g_{1}+\mathbf{j} g_{2}$. The twistor lift of $L^{\sharp}$ can be extended continuously through $p$, because the limit of $\psi^{\sharp}\left(g_{3}+\mathbf{j} g_{4}\right) z^{-n}$ at $p$ exists and is not zero, where $z$ is a local holomorphic chart centered at $p$. The claim now follows from Riemann's removable singularity theorem.

6.8. Bryant spheres with smooth ends are 2-soliton spheres. Bryant spheres with smooth ends [5] are surfaces of mean curvature one in hyperbolic space that compactify to immersed spheres by adding points on the ideal boundary of hyperbolic space. In [19] it is shown that a Bryant surface is characterized by the existence of a totally umbilic Darboux transform, which is then the hyperbolic Gauss map; see also [5, Theorem 9]. In order to apply Theorem 6.2 it remains to check that the holomorphic section defining this Darboux transform extends smoothly through smooth Bryant ends.

Remark 6.9. It seems worthwhile to note that the characterization of a Bryant surface [19, 5] by the existence of a totally umbilic Darboux transform requires that both, the surface and its Darboux transform, take values in the same round 3sphere in $S^{4}=\mathbb{H} \mathbb{P}^{1}$. This holds automatically for the "classical" Darboux transform in the isothermic surface sense used in [19, 5], but not for the Darboux transform of [4] used in Section 6.1. 

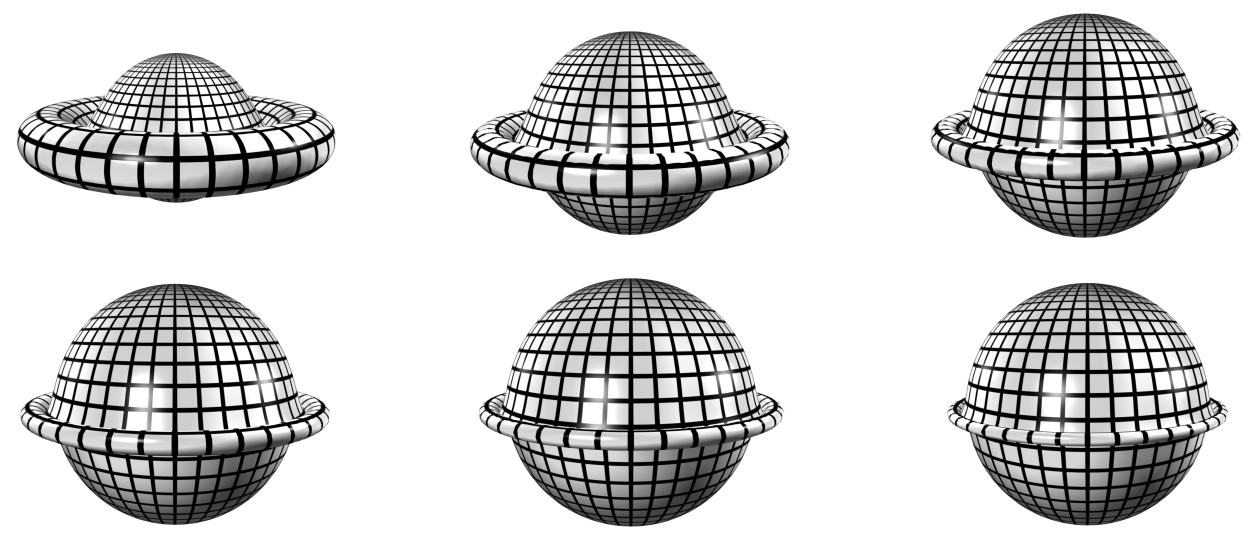

Figure 6. Catenoid cousins: $W=8 \pi(\mu+1), \mu=1,2,3,4,5,7$.

An immersion $L \subset \mathbb{H}^{2}$ of $\mathbb{C P}^{1}$ into $\mathbb{H}^{1}$ is a Bryant sphere with smooth ends if and only if, up to Möbius transformations,

$$
L=\psi \mathbb{H}, \quad \psi=F\left(\begin{array}{c}
\mathbf{k} \\
1
\end{array}\right),
$$

for a rational null immersion $F$ into $\mathrm{SL}(2, \mathbb{C})$ such that all poles of $d F F^{-1}$ have order 2 ; cf. [5. Here null immersion means that $\operatorname{det}(d F)=0$ and $d F$ has no zeros. The kernels and images of $d F F^{-1}$ then coincide and extend holomorphically through the poles of $F$. The holomorphic map

$$
L^{\sharp}=\operatorname{ker}\left(d F F^{-1}\right)=\operatorname{im}\left(d F F^{-1}\right)
$$

into the round 2-sphere $\{[x, 1] \mid x \in \mathbb{C}\} \cup\{[1,0]\} \subset \mathbb{H} \mathbb{P}^{1}$ is then called the hyperbolic Gauss map of $L$. The hyperbolic Gauss map $L^{\sharp}$ of a Bryant sphere $L$ with smooth ends extends through the ends to a rational map from $\mathbb{C P}^{1}$ to $\mathbb{C P}^{1}$.

Theorem 6.10 ([5]). Bryant spheres with smooth ends are 2-soliton spheres.

Proof. Denote by $\left\langle\left(\begin{array}{c}x_{1} \\ x_{2}\end{array}\right),\left(\begin{array}{l}y_{1} \\ y_{2}\end{array}\right)\right\rangle=\bar{x}_{2} \mathbf{j} y_{1}-\bar{x}_{1} \mathbf{j} y_{2}$ the indefinite Hermitian form whose null lines are the round 3-sphere $\left\{[x, 1] \mid x \in \operatorname{Span}_{\mathbb{R}}\{1, \mathbf{i}, \mathbf{k}\}\right\} \cup\{[1,0]\}$ in $\mathbb{H}^{1}$. Then $L=\psi \mathbb{H}, \psi=F\left(\begin{array}{l}\mathbf{k} \\ 1\end{array}\right)$ and $L^{\sharp}=\operatorname{ker}\left(d F F^{-1}\right)=\operatorname{im}\left(d F F^{-1}\right)$ as above are maps into this 3-sphere. Denote by $\psi^{\sharp}$ the section of $\Gamma\left(L^{\sharp}\right)$ defined away from the poles of $F$ by

$$
\left\langle\psi^{\sharp}, \psi\right\rangle=1 .
$$

The claim follows from Theorem 6.2 once we show that $\psi^{\sharp}$ is the prolongation of a holomorphic section of $\mathbb{H}^{2} / L$ that extends smoothly through the poles of $F$. Using that $d F$ takes values in the null lines $L^{\sharp}$, we obtain

$$
\left\langle\nabla \psi^{\sharp}, \psi\right\rangle=-\left\langle\psi^{\sharp}, \nabla \psi\right\rangle=-\left\langle\psi^{\sharp}, d F\left(\begin{array}{l}
\mathbf{k} \\
1
\end{array}\right)\right\rangle=0 .
$$

Hence $\nabla \psi^{\sharp}$ takes values in the null lines $L$, and $\psi^{\sharp}$ is the prolongation of the holomorphic section $\pi \psi^{\sharp}$ of $\mathbb{H}^{2} / L$. To see that $\pi \psi^{\sharp}$ extends smoothly through the poles of $F$, let $z$ be a local coordinate centered at a pole of $F$. Changing coordinates in $\mathbb{H}^{2}$ one may assume that $F=z^{-n}\left(\begin{array}{ll}a & b \\ c & d\end{array}\right)$ for some $n \in \mathbb{N}$ with holomorphic 


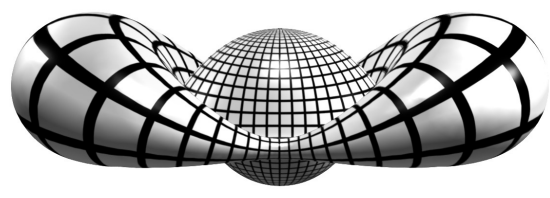

Figure 7. $\mu=2$, $W=16 \pi, s=0.22, t=$ 0 .

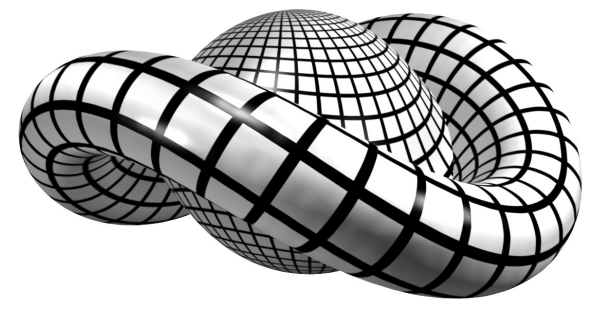

FiguRE 8. $\mu=2$, $W=16 \pi, s=0.22, t=$ 0.22 .

functions $a, b, c, d$ and $d(0) \neq 0$; see [5, Lemma 3]. Then

$$
\pi \psi^{\sharp}=\pi e_{1}\left(-\mathbf{j} \bar{z}^{n}(-c \mathbf{k}+\bar{d})^{-1}\right),
$$

which implies that the holomorphic section $\pi \psi^{\sharp}$ extends smoothly through the poles of $F$.

Example 6.11 (Catenoid cousins). The holomorphic null immersion

$$
[a, b, c, d, e]=\left[-\mu,(\mu+1) z^{2 \mu+1},-(\mu+1) z, \mu z^{2 \mu+2}, \sqrt{2 \mu+1} z^{\mu+1}\right]
$$

into the 3-quadric $Q^{3}=\left\{[a, b, c, d, e] \in \mathbb{C P}^{4} \mid a d-b c=e^{2}\right\}$ has as affine part the holomorphic null immersion $F=\frac{1}{e}\left(\begin{array}{ll}a & b \\ c & d\end{array}\right)$ into $\mathrm{SL}(2, \mathbb{C})$. The parameters $\mu>-1$, $\mu \neq 0$ yield, via $L=F\left(\begin{array}{c}\mathbf{k} \\ 1\end{array}\right) \mathbb{H}$, Bryant's catenoid cousins [8]. The ends of a catenoid cousin are smooth if and only if $\mu \in \mathbb{N} \backslash\{0\}$. Catenoid cousins with smooth ends are the simplest examples of Taimanov soliton spheres; cf. 4.10. Their Willmore energy is $W=8 \pi(\mu+1)$. Figure 6 shows $f: \mathbb{C P}^{1} \rightarrow \mathbb{R}^{3}=\operatorname{Im} \mathbb{H}$ defined by $\left[\begin{array}{l}f \\ 1\end{array}\right]=\left(\begin{array}{ll}\mathbf{j} & \mathbf{i} \\ \mathbf{k} & 1\end{array}\right) F\left(\begin{array}{l}\mathbf{k} \\ 1\end{array}\right) \mathbb{H}$ for different $\mu \in \mathbb{N}_{*}$.

Example 6.12 (Bryant spheres with arbitrarily many smooth ends). The two ends of a catenoid cousin have order $\mu+1$. Applying the transformation $(a, b, c, d, e) \mapsto$ $\left(a, b, c, s^{2} a+d-2 s e,-s a+e\right)$ followed by $(a, b, c, d, e) \mapsto\left(a+t^{2} d-2 t e, b, c, d,-t d+e\right)$ deforms each end to $\mu+1$ ends of order 1; see Figures 7,15

\section{BÄCKLUND TRANSFORMATION AND SOLITON SPHERES: \\ WILLMORE SPHERES}

We show that Willmore spheres in the conformal 4-sphere $S^{4}$ are examples of soliton spheres. The main idea of the proof is to use that a non-twistor holomorphic Willmore sphere admits a twistor holomorphic Bäcklund transformation.

7.1. Mean curvature sphere congruence. In the case of immersed holomorphic curves in $\mathbb{H}^{1}$, the canonical complex structure defined in Section 3.7 can be interpreted as the mean curvature sphere congruence.

The oriented totally umbilic 2 -spheres in the conformal 4 -sphere $\mathbb{H}^{1}$ are in one-to-one correspondence with the quaternionic linear complex structures on $\mathbb{H}^{2}$ : 

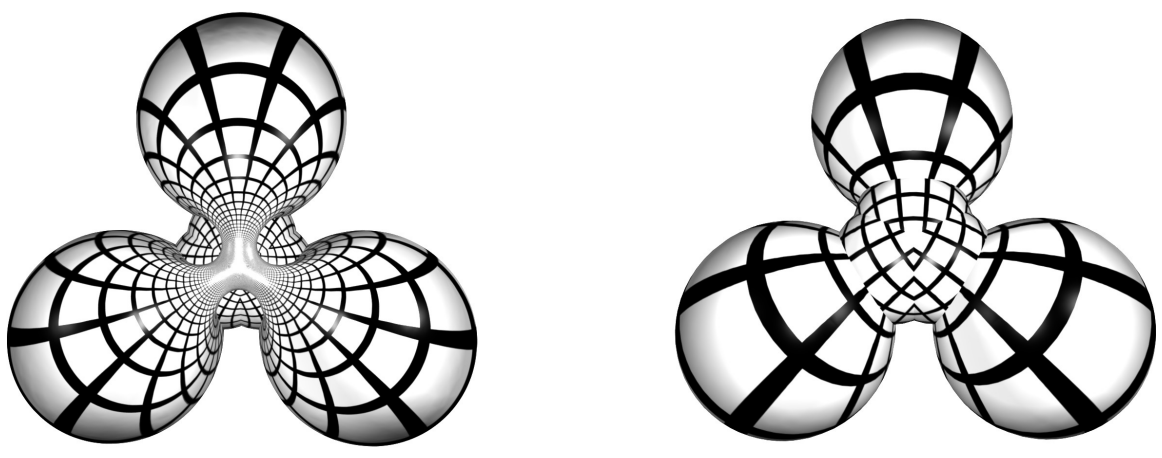

Figure 9. $\mu=3, W=24 \pi, s=2.3 \mathbf{i}, t=-0.33 \mathbf{i}$, two views.

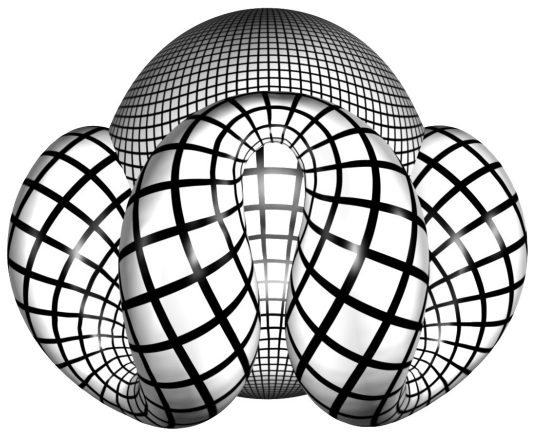

FiguRe 10. $\mu=4$,

$W=32 \pi, s=0.72 \mathbf{i}$,

$t=-0.54 \mathbf{i}$.

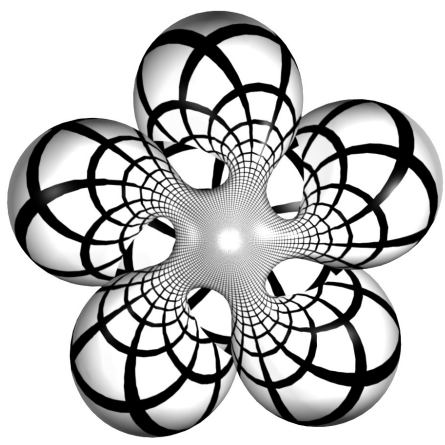

FiguRE 12. $\mu=5$,

$W=40 \pi, s=1.6 \mathbf{i}, t=$

-0.36 .

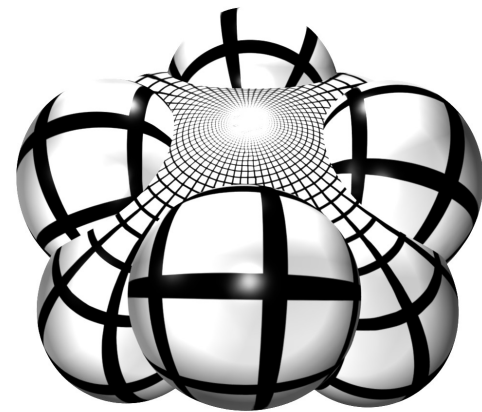

Figure 11. Möbius inversion of Figure 10,

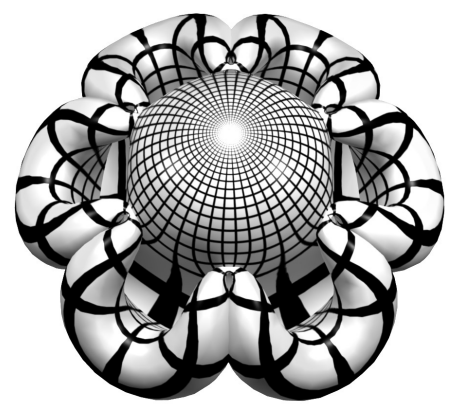

Figure 13. $\mu=6$, $W=48 \pi, s=0.22, t=$ -0.57 .

let $S \in \operatorname{End}\left(\mathbb{H}^{2}\right)$ such that $S^{2}=-1$; then

$$
\mathcal{S}=\left\{[x] \in \mathbb{H} \mathbb{P}^{1} \mid[S x]=[x]\right\}
$$




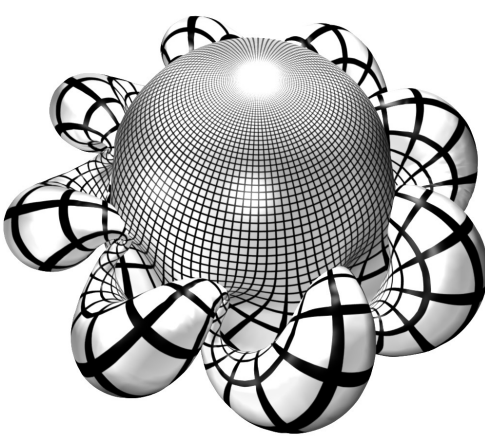

Figure 14. $\mu=8$, $W=64 \pi, s=-0.09-$ $0.24 \mathbf{i}, t=-0.42$.

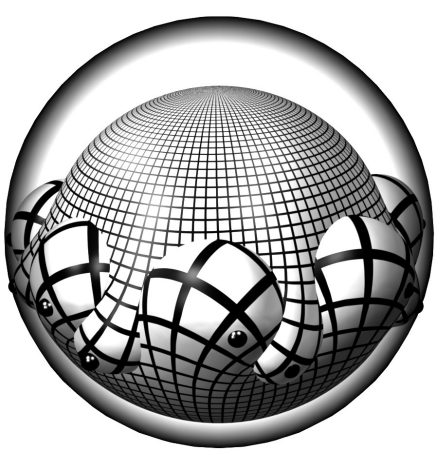

Figure 15. Möbius inversion of Figure 14 in Poincaré ball with marked ends.

is a totally umbilic 2 -sphere in $\mathbb{H} \mathbb{P}^{1}$. The complex structures $S$ and $-S$ define the same 2-sphere, but with different orientations on the tangent spaces $T_{p} \mathcal{S}=\mathbb{H}^{2} / p$, $p \in \mathcal{S}$. One therefore calls a map $S: M \rightarrow \operatorname{End}\left(\mathbb{H}^{2}\right)$ with $S^{2}=-1$ a sphere congruence. Its derivative may be decomposed as

$$
\nabla S=2(* Q-* A)
$$

into its $\bar{K}$-part $2 * Q$ and its $K$-part $-2 * A$ satisfying $* Q=-S Q=Q S$ and $* A=$ $S A=-A S$. The $\operatorname{End}\left(\mathbb{H}^{2}\right)$-valued 1-forms $A$ and $Q$ are called the Hopf fields of $S$. An immersed holomorphic curve $L \subset \mathbb{H}^{2}$ in $\mathbb{H} \mathbb{P}^{1}$ admits [10, Section 5] a unique sphere congruence satisfying

$$
S L=L, \quad * \delta=S \delta=\delta S, \quad L \subset \operatorname{ker}(Q) \quad(\text { or, equivalently, } \operatorname{im}(A) \subset L),
$$

which is called the mean curvature sphere congruence of $L$ and coincides with the canonical complex structure defined in Section 3.7 The name mean curvature sphere congruence reflects the fact that the sphere $S_{p}$ at a point $p \in M$ is the unique sphere that touches the curve at $L_{p}$ and has the same mean curvature vectors with respect to any compatible metric of the conformal 4 -sphere $S^{4}$; cf. [10, Section 5.2].

The Hopf fields $A$ and $Q$ measure the change of $S$ along the curve. The integrals $2 \int\langle A \wedge * A\rangle$ and $2 \int\langle Q \wedge * Q\rangle$ measure the global change of $S$ and coincide with the Willmore energies of the quaternionic holomorphic line bundles $L^{-1}$ and $\left(L^{\perp}\right)^{-1}=$ $\mathbb{H}^{2} / L$ which Kodaira correspond, as in Section 2.5, to $L$ and $L^{\perp}$, respectively.

It can be shown, e.g. [10, Section 6], that an immersed holomorphic curve in $\mathbb{H} \mathbb{P}^{1}$ is Willmore, i.e., a critical point of the Willmore energy, if and only if its mean curvature sphere is harmonic. This is equivalent to

$$
d * A=0, \quad \text { which is again equivalent to } \quad d * Q=0 .
$$

Special examples of Willmore surfaces are twistor holomorphic curves, which are characterized by $A \equiv 0$, see Lemma [3.8, and curves with $Q \equiv 0$ for which the dual curve $L^{\perp}$ is twistor holomorphic.

7.2. Willmore spheres in the 4-sphere. Bryant's classification [7] of Willmore spheres in the conformal 3-sphere has the following extension to the conformal 
4-sphere [12, 26, 25, 10]: an immersed Willmore sphere $L \subset \mathbb{H}^{2}$ in the conformal 4-sphere $S^{4}=\mathbb{H} \mathbb{P}^{1}$ is either

- twistor holomorphic, which is equivalent to $A \equiv 0$,

- its dual $L^{\perp}$ is twistor holomorphic, which is equivalent to $Q \equiv 0$,

- or it is Euclidean minimal,

where we call a holomorphic curve $L$ in $\mathbb{H}_{\mathbb{P}^{1}}$ Euclidean minimal if it is minimal in the Euclidean space $\mathbb{H}=\mathbb{H} \mathbb{P}^{1} \backslash\{\infty\}$ for some point $\infty \in \mathbb{H} \mathbb{P}^{1}$. This is equivalent to the Möbius invariant condition that all mean curvature spheres of $L$ intersect in one point. If a Euclidean minimal curve in $\mathbb{H}^{1}$ is immersed, the corresponding minimal immersion into $\mathbb{H}=\mathbb{H} \mathbb{P}^{1} \backslash\{\infty\}$ has planar ends [7] at the points where the curve goes through $\infty$.

Theorem 3.5 immediately implies that the first two cases are soliton spheres with equality in the Plücker estimate for the canonical linear system: if $A \equiv 0$, then $L$ itself is twistor holomorphic and equality in the Plücker estimate holds for the canonical linear system of $\left(L^{\perp}\right)^{-1}=\mathbb{H}^{2} / L$. If $Q \equiv 0$, then $L^{\perp}$ is twistor holomorphic and equality holds for the canonical linear system of $L^{-1}=\mathbb{H}^{2} / L^{\perp}$. It therefore remains to show that Euclidean minimal spheres are soliton spheres.

7.3. Euclidean minimal curves. Let $L \subset \mathbb{H}^{2}$ be an immersed Euclidean minimal curve with mean curvature sphere congruence $S$ and $\infty=[x] \in \mathbb{H}^{1}$ the point contained in all mean curvature spheres. Then $\left[S_{p} x\right]=[x]$ for all $p \in M$ such that $\nabla S x$ takes values in the subspace $[x] \subset \mathbb{H}^{2}$. Using the type decomposition $\nabla S=2 * Q-2 * A$, this implies

$$
\operatorname{im}(* Q) \subset[x] \subset \operatorname{ker}(* A),
$$

because $L \subset \operatorname{ker}(Q), \operatorname{im}(A) \subset L$ and $[x]=L_{p}$ at isolated $p \in M$ only. In particular $L$ is Willmore, because $d * Q=0$, which follows from $d * Q x=\frac{1}{2} d(\nabla S) x=0$ and $d * Q \psi=* Q \wedge \delta \psi=0$ for all $\psi \in \Gamma(L)$.

Theorem 7.4. Immersed Willmore spheres in $\mathbb{H} \mathbb{P}^{1}$ are soliton spheres.

Proof. As seen in Section 7.2 it suffices to show that every immersed Euclidean minimal sphere $L \subset \mathbb{H}^{2}$ whose Hopf field $A$ does not vanish identically is a soliton sphere. We fix a point $\infty=\left[e_{1}\right] \in \mathbb{H}^{1}$ such that $L$ does not go through $\infty$ and, using the notation of Section [5.2, write $L=\psi \mathbb{H}$ with $\psi=\left(\begin{array}{l}f \\ 1\end{array}\right)$ for $f: \mathbb{C P}^{1} \rightarrow \mathbb{H}$. Then $f: \mathbb{C P}^{1} \rightarrow \mathbb{H}$ is not minimal in the Euclidean space $\mathbb{R}^{4}=\mathbb{H}$.

Because $\mathbb{C P}^{1}$ is simply connected, there is a globally defined 1-step forward Bäcklund transform $g: \mathbb{C P}^{1} \rightarrow \mathbb{H}$ of $f$ that satisfies

$$
d g=e_{2}^{*}\left(2 * A e_{1}\right)
$$

(see Appendix B.2). It is non-constant, because $f$ is not Euclidean minimal. By assumption there is $a \in \mathbb{H}$ such that $(f-a)^{-1}$ is Euclidean minimal. Theorem B.4(iii) thus implies that $g$ is twistor holomorphic. By Theorem 3.5 this yields that the linear system $H=\operatorname{Span}\{\psi, \psi g\} \subset H^{0}(L)$ of the Euclidean holomorphic structure on $L$ defined by $\infty$ has equality in the Plücker estimate. Hence $L$ is a soliton sphere by Theorem 5.3 .

Using Proposition 3.12 and Corollaries B.5 and B.6, the proof of Theorem 7.4 gives rise to the following representation of Willmore spheres in the conformal 3and 4-sphere in terms of twistor holomorphic curves. Appendix B.7 explains how this representation is related to the Weierstrass representation of minimal surfaces. 
Corollary 7.5. Let $f: \mathbb{C P}^{1} \rightarrow \mathbb{H}$ be a conformally immersed sphere. Suppose that neither $f$ nor $\bar{f}$ is twistor holomorphic. Then $f$ is Willmore if and only if there is a twistor holomorphic curve $L: \mathbb{C P}^{1} \rightarrow \mathbb{H}^{1}$ with smoothly immersed mean curvature sphere congruence $S$ such that

$$
f=e_{2}^{*}\left(S e_{1}\right)+c,
$$

for some $c \in \mathbb{H}, e_{1} \in \mathbb{H}^{2} \backslash\{0\}$, and $e_{2}^{*} \in\left(\mathbb{H}^{2}\right)^{*} \backslash\{0\}$ such that $e_{2}^{*}\left(e_{1}\right)=0$.

The Willmore sphere $f$ takes values in $\mathbb{R}^{3}=\operatorname{Im} \mathbb{H}$ if and only if the twistor holomorphic curve $L$ is hyperbolic superminimal with respect to the hyperbolic geometry defined by the Hermitian form $\left\langle\left(\begin{array}{c}x_{1} \\ x_{2}\end{array}\right),\left(\begin{array}{l}y_{1} \\ y_{2}\end{array}\right)\right\rangle=\bar{x}_{2} y_{1}+\bar{x}_{1} y_{2}$ (see Appendix C.9) and

$$
f=\langle a, S a\rangle+c
$$

for some $c \in \operatorname{Im} \mathbb{H}$ and $a \in \mathbb{H}^{2} \backslash\{0\}$ with $\langle a, a\rangle=0$.

Remark 7.6. The Willmore energy of a Willmore sphere $f$ obtained as in Corollary 7.5 from a twistor holomorphic curve $L$ is

$$
W(f)=4 \pi(2 d-2-b),
$$

where $d=-\operatorname{deg}(L)$ is the degree of $L$ and $b$ its branching degree; cf. the proof of Lemma 8.5. If $f$ takes values in $\operatorname{Im}(\mathbb{H})$, then $b=d-3$ and hence $W(f)=4 \pi(d+1)$.

Example 7.7 (Willmore spheres in $S^{3}$ with Willmore energy $16 \pi$ ). As an application of Corollary 7.5 we derive a formula for Willmore spheres in the conformal 3 -sphere with Willmore energy $16 \pi$, the lowest critical value of the Willmore energy for spheres in $S^{3}$ above the minimum $4 \pi$.

Remark 7.6 implies that $d=3$ and $b=0$ for Willmore spheres in $S^{3}$ with Willmore energy $16 \pi$. By PropositionC.11, the twistor projection $L$ of the holomorphic curve $\hat{L}=[\varphi]: \mathbb{C P}^{1} \rightarrow \mathbb{C P}^{3}$ given by

$$
\varphi:=e_{1} z+e_{1} \mathbf{j} \frac{1}{6} z^{3}-e_{2}+e_{2} \mathbf{j} \frac{1}{2} z^{2}
$$

is hyperbolic minimal with respect to the Hermitian form in Corollary 7.5 , because in the basis $\hat{e}_{1}, \ldots, \hat{e}_{6}$ of Appendix B.7 the curve $\hat{L}$ has the tangent line congruence $\hat{L}_{1}=[\hat{S}]: \mathbb{C P}^{1} \rightarrow Q^{4}=\left\{[v] \in P\left(\Lambda^{2}\left(\mathbb{H}^{2}, \mathbf{i}\right)\right) \mid v \wedge v=0\right\}$ given by

$$
\hat{S}=\varphi \wedge \varphi^{\prime}=\frac{1}{24}\left(0,-12 \mathbf{i} z^{2}, 12-z^{4},-12 \mathbf{i}-\mathbf{i} z^{4}, 8 z^{3},-24 z\right),
$$

which is polar to the space like vector $\hat{e}_{1}$.

Figure[16] shows the Willmore spheres $f=\langle a, S a\rangle$ in $\mathbb{R}^{3}$ obtained for $a=e_{2}+e_{2} \mathbf{j}$ (left) and $a=-e_{1}+e_{2}$ (right). The left image in Figure 17 shows $f=\langle a, S a\rangle$ with $\varphi$ replaced by $\varphi+e_{2} \mathbf{j} 3 z$ and $a=e_{2}+e_{2} \mathbf{j}$; the right image is obtained for $a=-e_{1}+e_{2}$ when $\varphi$ is replaced by $\varphi+e_{2} \mathbf{j} 7 z$.

\section{Willmore NUMBERS OF SOLITON SPHERES IN 3-SPACE}

In [9] Bryant shows that the possible Willmore energies $W=\int|\mathcal{H}|^{2}$ of Willmore spheres in $\mathbb{R}^{3}$ are $W=4 \pi d$ with $d \in(\mathbb{N} \backslash\{0,2,3,5,7\})$. The same quantization holds for Bryant spheres with smooth ends [5] and for Taimanov soliton spheres; see Corollary 4.9. In the present section we show that this quantization more generally holds for all immersed soliton spheres in 3-space. The main ingredient in the proof of this is Theorem 8.11, which says that all soliton spheres in 3-space with Willmore energy $W \leq 32 \pi$ are Willmore spheres or Bryant spheres with smooth ends. 

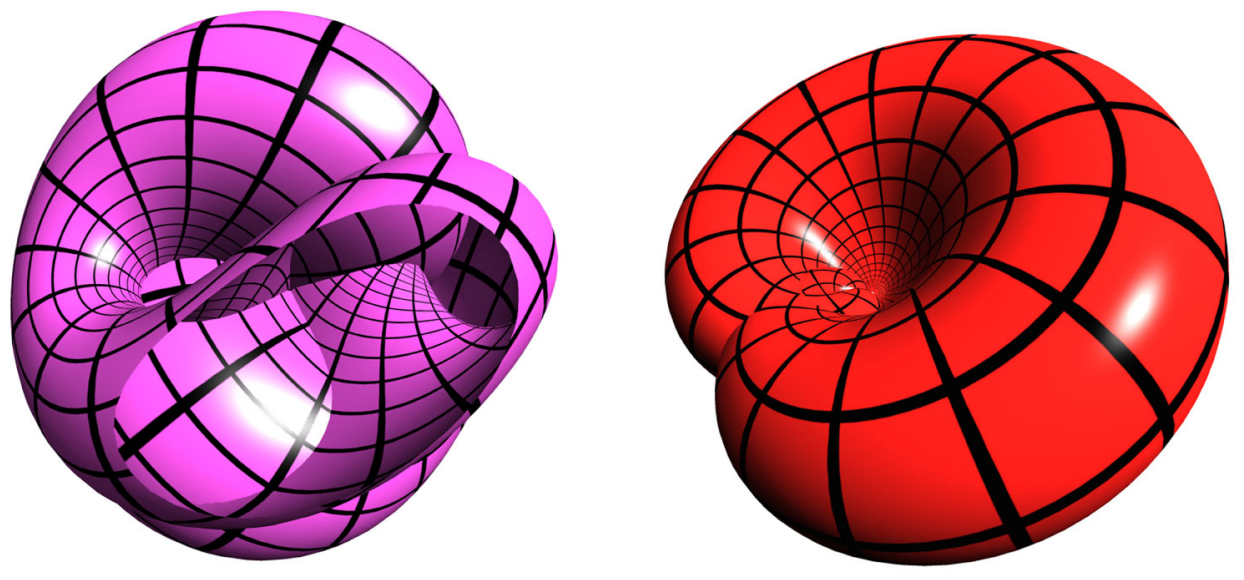

FiguRE 16. Willmore spheres with Willmore energy $16 \pi$.
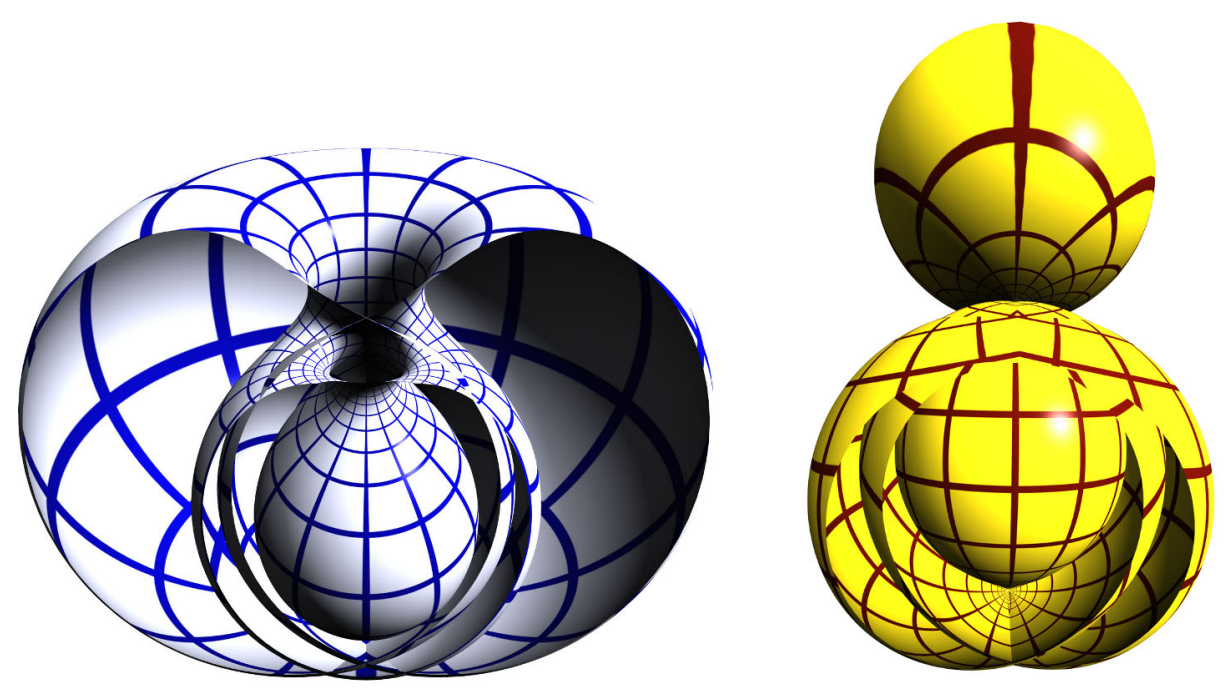

FiguRE 17 . Willmore spheres with Willmore energy $16 \pi$.

8.1. Equality in the Plücker estimate for spin bundles over $\mathbb{C P}^{1}$. In order to investigate soliton spheres in the conformal 3-sphere we apply the characterization in terms of Euclidean holomorphic line bundles given in Section 5.2. The advantage of the Euclidean point of view is that, if a conformal immersion $L \subset \mathbb{H}^{2}$ into $\mathbb{H}^{1}$ takes values in a totally umbilic 3 -sphere $S^{3} \subset \mathbb{H P}^{1}$, the Euclidean holomorphic structure on $L$ defined by a point $\infty \in S^{3}$ not on $L$ makes $L$ into a quaternionic spin bundle; see Section 4.3 .

Let $L=\psi \mathbb{H}, \psi=\left(\begin{array}{c}f \\ 1\end{array}\right)$ with affine representation $f: \mathbb{C P}^{1} \rightarrow \operatorname{Im} \mathbb{H}$ and $\infty=\left(\begin{array}{l}1 \\ 0\end{array}\right) \mathbb{H}$. As explained in Section 5.2, the Euclidean holomorphic line bundle corresponding to $\infty$ is $L$ equipped with the unique holomorphic structure for which $\psi$ is holomorphic. 
Since $f$ takes values in $\operatorname{Im}(\mathbb{H})$, this quaternionic holomorphic line bundle $L$ is spin, i.e., $K L^{-1} \cong L$, and

$$
d f=(\psi, \psi)
$$

see Theorem 4.4. The Willmore energy of the Euclidean holomorphic structure on $L$ is then $W(L)=\int|\mathcal{H}|^{2}$; see Section 4.1. In the following we call $W(L)$ the Willmore energy of a soliton sphere in the conformal 3-sphere. Its relation to the Willmore energy of the Möbius invariant holomorphic line bundle $\mathbb{H}^{2} / L$ is $W(L)=W\left(\mathbb{H}^{2} / L\right)+4 \pi ;$ see Section 2.7

By Theorem 5.3 the conformal immersion $f$ is a soliton sphere if and only if the spin bundle $L$ admits a base point free linear system $H \subset H^{0}(L)$ that contains $\psi$ and has equality in the Plücker estimate. Equality in the Plücker estimate for an $(n+1)$-dimensional linear system $H \subset H^{0}(L)$ of a quaternionic spin bundle $L$ over $\mathbb{C P}^{1}$ reads

$$
W(L)=4 \pi\left[(n+1)^{2}+\mid \text { ord } H \mid\right]
$$

because $K L^{-1} \cong L$ and hence $\operatorname{deg}(L)=\frac{1}{2} \operatorname{deg}(K)=-1$.

8.2. The possible gaps in the sequence of Willmore numbers. Examples of soliton spheres in the conformal 3-sphere with Willmore energy $W(L) \in$ $4 \pi(\mathbb{N} \backslash\{0,2,3,5,7\})$ can be found in each of the special classes of soliton spheres discussed in Sections 4 6, and 7 that is, among immersed Taimanov soliton spheres, Corollary 4.9, Bryant spheres with smooth ends [5], and Willmore spheres in the conformal 3-sphere; see [9]. It is therefore sufficient to show that $W \in 4 \pi\{2,3,5,7\}$ does not occur as Willmore energy of immersed soliton spheres in 3-space.

Lemma 8.3. The only soliton sphere in 3-space with $W(L)<16 \pi$ is the round sphere for which $W(L)=4 \pi$.

Proof. If $W(L)<16 \pi$, then $n=0$ and the linear system $H \subset H^{0}(L)$ above is 1 -dimensional. Because it is base point free, it has no Weierstrass points. Thus $W(L)=4 \pi$, which implies that the immersion is the round sphere [37.

This shows that the Willmore energies $8 \pi$ and $12 \pi$ do not occur so that it remains to check that $20 \pi$ and $28 \pi$ are impossible.

8.4. Soliton spheres in 3-space related to superminimal curves. If $L$ is the spin bundle of a soliton sphere in 3 -space with $16 \pi \leq W(L) \leq 32 \pi$, then the base point free linear system $H$ with equality in the quaternionic Plücker estimate in Section 8.1 is 2-dimensional and "full" in the sense that $H=H^{0}(L)$. In particular we are in the situation described by Proposition 3.12 i.e., the dual curve $L^{d}$ of $H \cong \mathbb{H}^{2}$ is twistor holomorphic, extends through the Weierstrass points of $H$, and has a mean curvature sphere congruence which is everywhere defined and smooth.

Lemma 8.5. Let $L^{d} \subset \mathbb{H}^{2}$ be a twistor holomorphic curve over $\mathbb{C P}^{1}$ with mean curvature sphere congruence that is everywhere defined and smooth. Then $L \cong$ $\mathbb{H}^{2} / L^{d}$ has the degree $\operatorname{deg}(L)=-1$ of a spin bundle if and only if

$$
\left|b\left(L^{d}\right)\right|=d-3
$$

where $\left|b\left(L^{d}\right)\right|$ is the branching order and $d=-\operatorname{deg}\left(L^{d}\right)$ the degree of the holomorphic curve $L^{d}$. The Willmore energy $W(L)$ of $L$ is then

$$
W(L)=4 \pi(d+1) \text {. }
$$


Proof. Because the derivative $\delta: L^{d} \rightarrow K L$ of $L^{d}$ is complex holomorphic [10, Section 13.2] one gets

$$
\left|b\left(L^{d}\right)\right|=\operatorname{deg}(K L)-\operatorname{deg}\left(L^{d}\right) .
$$

Hence, $\operatorname{deg}(L)=-1$ if and only if $\left|b\left(L^{d}\right)\right|=d-3$. By Proposition A.2 the Weierstrass order of $H=H^{0}(L)$ coincides with the branching order $b\left(L^{d}\right)$ of $L^{d}$. Equality in the Plücker estimate for $H$ thus yields

$$
W(L)=4 \pi\left[4+\left|b\left(L^{d}\right)\right|\right] .
$$

In order to derive a condition on the dual curve $L^{d}$ which guarantees that $L$ is spin, i.e., $K L^{-1} \cong L$, we make use of a bundle isomorphism induced by $L^{d}$ : let $L^{d} \subset H \cong \mathbb{H}^{2}$ be a twistor holomorphic curve with everywhere defined and smooth mean curvature sphere congruence $S$; cf. Proposition 3.12, Assuming that the Willmore energy $W(L)$ of $L \cong H / L^{d}$ is not zero, the Hopf field $Q$ of $L^{d}$ does not vanish identically and defines a unique holomorphic curve

$$
\tilde{L} \subset \operatorname{ker}\left(Q^{*}\right) \subset H^{*}
$$

called the 2-step forward Bäcklund transformation of $\left(L^{d}\right)^{\perp}$; see Appendix B Because $L^{d} \subset \operatorname{ker}(Q)$ and $* Q=-S Q=Q S$, the bundle homomorphism $* Q^{*}$ maps the trivial $H^{*}$-bundle to the bundle $K L^{-1}$ of $(1,0)$-forms with values in $L^{-1} \cong\left(L^{d}\right)^{\perp} \subset H^{*}$. This gives rise to a quaternionic line bundle homomorphism

$$
* Q^{*}: H^{*} / \tilde{L} \rightarrow K L^{-1} \quad(x \bmod \tilde{L}) \mapsto * Q^{*} x,
$$

which is complex linear with respect to the complex structure on $H^{*} / \tilde{L}$ induced by $-S^{*}$ and the usual complex structure induced by $S^{*}$ on $L^{-1} \cong\left(L^{d}\right)^{\perp} \subset H^{*}$.

Lemma 8.6. Let $L^{d} \subset H \cong \mathbb{H}^{2}$ be a twistor holomorphic curve with everywhere defined and smooth mean curvature sphere congruence $S$ and non-trivial $Q$. Denote by $\tilde{L} \subset \operatorname{ker}\left(Q^{*}\right)$ the 2-step Bäcklund transform of $\left(L^{d}\right)^{\perp}$. Then $* Q^{*}: H^{*} / \tilde{L} \rightarrow$ $K L^{-1}$ is a holomorphic bundle homomorphism, where $K L^{-1}$ is equipped with the holomorphic structure paired with that on $L=H / L^{d}$ (Section 4.1) and $H^{*} / \tilde{L}$ is equipped with the unique holomorphic structure with respect to which all projections of constant sections of $H^{*}$ are holomorphic and whose complex structure is induced by $-S^{*}$.

Proof. In order to check that the above holomorphic structure on $H^{*} / \tilde{L}$ is well defined we have to distinguish two cases: if $\tilde{L}$ is constant, the holomorphic structure is given by $D=\nabla^{\prime \prime}=\frac{1}{2}(\nabla+* J \nabla)$, where $J$ denotes the complex structure on $H^{*} / \tilde{L}$ induced by $-S^{*}$ and $\nabla$ the trivial connection of $H^{*} / \tilde{L}$. If $\tilde{L}$ is non-constant, then $H^{*} / \tilde{L}$ is the canonical holomorphic line bundle of the holomorphic curve $\tilde{L}^{\perp}$ as defined in Section 2.5, because $0=\left.d * Q^{*}\right|_{\tilde{L}}=-* Q^{*} \wedge \tilde{\delta}$ implies $\tilde{\delta}=-S^{*} \tilde{\delta}$. Holomorphicity of the bundle homomorphism induced by $* Q^{*}$ also follows from $d * Q=0$ : by definition of the holomorphic structure on $K L^{-1}$, a section $\alpha \in$ $\Gamma\left(K L^{-1}\right)$ is holomorphic if and only if $\alpha(a)$ is closed for all $a \in H$. But $* Q^{*} y \in$ $\Gamma\left(K L^{-1}\right)$ is closed for every $y \in H^{*}$ and hence $* Q^{*}$ maps the projection to $H^{*} / \tilde{L}$ of every constant section of $H^{*}$ to a holomorphic section of $K L^{-1}$; cf. Section 4.1 , 
Now assume that $L$ is a spin bundle, i.e., $K L^{-1} \cong L$. The composition of the bundle homomorphism $2 * Q^{*}$ with the isomorphism $K L^{-1} \cong L$ is then a holomorphic bundle homomorphism

$$
B: H^{*} / \tilde{L} \rightarrow L
$$

The image $B\left(H^{*}\right) \subset H^{0}(L)$ under $B$ of the space of sections of $H^{*} / \tilde{L}$ obtained by projecting constant sections of $H^{*}$ is a 1 - or 2-dimensional subspace of $H^{0}(L)$ depending on whether $\tilde{L}$ is constant or non-constant.

Remark 8.7. One can show that if $H$ has equality in the Plücker estimate, then $B\left(H^{*}\right)$ has equality and is either contained in the canonical linear system $H \subset$ $H^{0}(L)$ of the curve $L^{d}$ or $H \cap B\left(H^{*}\right)=\{0\}$ and $B\left(H^{*}\right)$ is 2-dimensional.

The following proposition characterizes the twistor holomorphic curves $L^{d} \subset$ $H \cong \mathbb{H}^{2}$ with smooth $S$ and non-trivial $Q$ for which $L=H / L^{d}$ is spin and $B\left(H^{*}\right)$ is contained in the canonical linear system $H \subset H^{0}(L)$ of $L^{d}$. Note that if $W(L) \leq$ $32 \pi$ the Plücker estimate implies $B\left(H^{*}\right) \subset H$, because then $H=H^{0}(L)$.

Proposition 8.8. Let $L$ be a quaternionic holomorphic line bundle over $\mathbb{C P}^{1}$ and $H \subset H^{0}(L)$ a base point free, 2-dimensional linear system with equality in the Plücker estimate and dual curve $L^{d} \subset H \cong \mathbb{H}^{2}$. Then $L$ is a quaternionic spin bundle with $B\left(H^{*}\right) \subset H$ if and only if either

a) $L^{d}$ is spherical or hyperbolic superminimal with everywhere defined and immersed mean curvature sphere congruence, or

b) $L^{d}$ is Euclidean superminimal in $\mathbb{H}^{1} \backslash\{\infty\}$ for some point $\infty \in \mathbb{H} \mathbb{P}^{1}$ and the intersection divisor of $L^{d}$ with $\infty$ is equal to the branching divisor of the globally defined mean curvature sphere congruence of $L^{d}$.

Remark 8.9. By Proposition C.11, the tangent line congruence $\widehat{L_{1}^{d}}$ of the twistor lift $\widehat{L^{d}}$ of a curve $L^{d}$ belonging to type 国) is a rational null immersion into the complex 3-quadric. Denote by $d$ and $d_{1}$ the degrees of $L^{d}$ and $\widehat{L_{1}^{d}}$. Using that $\widehat{L^{d}}$ is self-dual, see Remark[C.12, the complex Plücker Formula [17, p. 270] for $\widehat{L_{1}^{d}}$ implies $0=-2 d+2 d_{1}-2$, because $\widehat{L_{1}^{d}}$ is unbranched if $S$ is immersed; see Lemma C.8. By Lemma 8.5 we thus have $\frac{1}{4 \pi} W(L)=d+1=d_{1}$. This excludes type 国) curves for which $W(L)=20 \pi$ or $W(L)=28 \pi$, because rational null immersions of degree 5 and 7 into the complex 3 -quadric do not exist; cf. 9].

To prove the quantization of the Willmore energy for soliton spheres in 3-space it therefore remains to exclude type (b) curves of degrees 4 and 6 . A direct proof of this turns out to be very technical. Below such curves are excluded by a Möbius geometric argument; see the proof of Theorem 8.11.

Proof. From Proposition 3.12 we know that a base point free, 2-dimensional linear system $H \subset H^{0}(L)$ with equality in the Plücker estimate has a globally defined, twistor holomorphic dual curve $L^{d} \subset H$ whose mean curvature sphere congruence $S$ extends smoothly through its branch points.

Assume that $L$ is spin and $B\left(H^{*}\right) \subset H$. We distinguish the cases of non-constant and constant Bäcklund transforms $\tilde{L} \subset \operatorname{ker}\left(Q^{*}\right)$.

(a) If $\tilde{L}$ is non-constant, then $B$ maps the space $H^{*}$ of holomorphic sections of $H^{*} / \tilde{L}$ onto $H \subset H^{0}(L)$. Because by assumption $H$ is base point free, the bundle 
homomorphism $B$ is an isomorphism. Since $\nabla S=2 * Q$, Lemma 3.8, the mean curvature sphere congruence $S$ of $L^{d}$ is immersed.

The bundle isomorphism $B$ induces an isomorphism $\tilde{B}: H^{*} \rightarrow H$ of vector spaces between the 2-dimensional linear systems $H^{*}$ and $H$. It maps $\tilde{L} \subset H^{*}$ onto $L^{d}$ and thus maps the mean curvature sphere congruence $-S^{*}$ of $\tilde{L}$ onto the mean curvature sphere congruence $S$ of $L$, i.e., $S \tilde{B}=-\tilde{B} S^{*}$. Differentiating the last equation one obtains that $\tilde{B}$ maps $\left(L^{d}\right)^{\perp}=\operatorname{im}\left(Q^{*}\right)$ onto $\tilde{L}^{\perp}=\operatorname{im}(Q)$. This implies that the adjoint $\tilde{B}^{*}$ of $\tilde{B}$ also induces a bundle isomorphism $H^{*} / \tilde{L} \rightarrow L \cong H / L^{d}$. Because every automorphism of a quaternionic holomorphic line bundle with nontrivial Hopf field acts by multiplication with a real constant, we obtain that $\tilde{B}^{*}=$ $\pm \tilde{B}$. Now $\tilde{B}^{*}=-\tilde{B}$ is impossible: for every $x \in H^{*} \backslash\{0\}$ the non-trivial 1-form $\left\langle * Q^{*} x, \tilde{B} x\right\rangle=* Q^{*} x(B x)$ had to be real-valued, because $S \tilde{B}=-\tilde{B} S^{*}$ implies $Q \tilde{B}=-\tilde{B} Q^{*}$ and hence

$$
\overline{\left\langle * Q^{*} x, \tilde{B} x\right\rangle}=\left\langle x, \tilde{B}^{*} * Q^{*} x\right\rangle=-\left\langle x, \tilde{B} * Q^{*} x\right\rangle=\langle x, * Q \tilde{B} x\rangle=\left\langle * Q^{*} x, \tilde{B} x\right\rangle .
$$

But this contradicts $*\left\langle * Q^{*} x, \tilde{B} x\right\rangle=-\left\langle * Q^{*} S^{*} x, \tilde{B} x\right\rangle=N\left\langle * Q^{*} x, \tilde{B} x\right\rangle$ with $N \in$ $\operatorname{Im} \mathbb{H}$ defined by $S^{*} x=x N \bmod \tilde{L}$. Hence $\tilde{B}^{*}=\tilde{B}$ and $\tilde{B}$ defines a non-degenerate Hermitian form on $H$ with respect to which $S$ is skew. Thus $L$ is spherical or hyperbolic superminimal, by Lemma C.10.

(b) If $\tilde{L}$ is constant, then $\infty=\tilde{L}^{\perp} \subset H$ lies on all mean curvature spheres of $L^{d}$. Hence $L^{d}$ is Euclidean superminimal. The statement about the intersection divisor may be seen as follows: the vanishing divisor of a non-trivial holomorphic section $x \in H \subset H^{0}(L)$ with $x \in \infty$ is the intersection divisor of $L^{d}$ with $\infty$. Let $y \in H^{*}$ with $y(x)=1$. Because $y \notin \tilde{L}=\infty^{\perp}$ it induces a nowhere-vanishing holomorphic section of $H^{*} / \tilde{L}$. The vanishing divisor of $B y$ thus equals the branching divisor of $S$, since $\nabla S=2 * Q$.

We now prove that the holomorphic sections $B y$ and $x$ differ by a real constant only and hence have the same vanishing divisor. Because $S$ is smooth and $S \infty=\infty$, there is a map $N: \mathbb{C P}^{1} \rightarrow S^{2}$ with $S x=x N$. On the other hand, since $y(x)=1$ we have $-S^{*} y \equiv y N \bmod \tilde{L}$ and $S(B y)=(B y) N$. Thus, away from the isolated zeros of $B y$ and $x$ there exist real-valued functions $\lambda_{1}, \lambda_{2}$ with $B y=x\left(\lambda_{1}+\lambda_{2} N\right)$. Since $Q$ is non-trivial, the Leibniz rule in Section 2.2 implies that $\lambda_{1}$ is constant and $\lambda_{2} \equiv 0$. Hence $B y=x \lambda_{1}$ with $\lambda_{1} \in \mathbb{R}$ such that $B y$ and $x$ have the same vanishing divisor.

We now show that, conversely, if $L^{d} \subset H$ is a superminimal curve as in (a) or $(\mathrm{b})$, then $L=H / L^{d}$ is a quaternionic spin bundle, i.e., $K L^{-1} \cong L$, which by construction satisfies $B\left(H^{*}\right) \subset H$.

(a) Let $L^{d}$ be spherical or hyperbolic superminimal. Then there exists a nondegenerate Hermitian form $\langle\cdot, \cdot\rangle$ with respect to which $S=-S^{*}$, see Lemma C.10, and $\nabla S=2 * Q$ because $L^{d}$ is twistor holomorphic. Identifying $H$ and $H^{*}$ via $\langle\cdot, \cdot\rangle$, this implies $Q=-Q^{*}$ and hence $\tilde{L}=\operatorname{ker}\left(Q^{*}\right)=\operatorname{ker}(Q)=L^{d}$. Since $\operatorname{im}(Q)=\tilde{L}^{\perp}=\left(L^{d}\right)^{\perp} \cong\left(H / L^{d}\right)^{-1}=L^{-1}$, the Hopf field $* Q$ induces a quaternionic holomorphic bundle homomorphism $L \rightarrow K L^{-1}$, which is an isomorphism because by assumption $S$ is an immersion.

(b) If $L^{d}$ is Euclidean superminimal, then $\infty=\tilde{L}^{\perp}=\operatorname{im}(Q)$ is a constant point. As above, let $x \in \infty$ and $y \in\left(\mathbb{H}^{2}\right)^{*}$ such that $y(x)=1$. Then $* Q^{*} y$ is a holomorphic section of $K L^{-1}$ whose vanishing divisor by assumption coincides with the vanishing 
divisor of $x$ seen as a holomorphic section of $L$. As before, there exists a quaternionvalued map $N$ defined away from the zeros of $x$ and $* Q^{*} y$, such that $J x=x N$ and $J * Q^{*} y=* Q^{*} y N$, where $J$ denotes the complex structures of the respective quaternionic holomorphic line bundles $L$ and $K L^{-1}$. This proves $L \cong K L^{-1}$, because the identification of two holomorphic sections with the same vanishing divisor and the same "normal vector" $N$ gives rise to a holomorphic line bundle isomorphism. This follows from Riemann's removable singularity theorem, because holomorphic homomorphisms between two quaternionic holomorphic line bundles are in particular complex holomorphic sections of the line bundle of homomorphisms between the underlying complex holomorphic line bundles.

8.10. Classification of soliton spheres in 3-space with Willmore energy $16 \pi \leq W \leq 32 \pi$. Let $L \subset \mathbb{H}^{2}$ be a soliton sphere with $16 \pi \leq W \leq 32 \pi$ in the conformal 3-sphere $S^{3} \subset \mathbb{H} \mathbb{P}^{1}$. For every $\infty \in S^{3}$ not on $L$, the induced quaternionic spin structure on $L$ admits a 2 -dimensional linear system related to a superminimal curve as described in Proposition 8.8. Investigation of the corresponding 3 -dimensional linear system of the Möbius invariant holomorphic line bundle $\mathbb{H}^{2} / L$ shows that $L$ is either a Willmore sphere or a Bryant sphere with smooth ends.

Theorem 8.11. Let $L \subset \mathbb{H}^{2}$ be a soliton sphere in the conformal 3-sphere with Willmore energy $16 \pi \leq W \leq 32 \pi$. Then the full space of holomorphic sections $H^{0}\left(\mathbb{H}^{2} / L\right)$ of the Möbius invariant holomorphic line bundle $\mathbb{H}^{2} / L$ is a 3-dimensional linear system with equality in the Plücker estimate that also contains a (unique) 1-dimensional linear system $H \subset H^{0}\left(\mathbb{H}^{2} / L\right)$ with equality in the Plücker estimate. Moreover, either

- L is a Willmore sphere or

- L is a Bryant sphere with smooth ends,

depending on whether $H$ is contained in the canonical linear system or not. In the Bryant case, the Darboux transform corresponding to $H$ is the hyperbolic Gauss map of L. In the Willmore case, the Darboux transform is constant and coincides with the point $\infty$ for which $L$ is Euclidean minimal in $\mathbb{H} \mathbb{P}^{1} \backslash\{\infty\}$.

Remark 8.12. The assumption $16 \pi \leq W \leq 32 \pi$ in Theorem 8.11 may be replaced by the weaker assumption $B\left(H^{*}\right) \subset H$ of Proposition 8.8. In the proof of Theorem 8.11 we show that a soliton sphere $L$ in $\mathbb{R}^{3}=\operatorname{Im}(\mathbb{H})$ whose spin bundle is of type a) in Proposition 8.8 is a Willmore sphere or a Bryant sphere with smooth ends (in the ball model of hyperbolic space). A soliton sphere in $\mathbb{R}^{3}$ whose spin bundle is of type b) in Proposition 8.8 is a Bryant sphere with smooth ends (in the half-space model of hyperbolic space).

For the proof of Theorem 8.11 we need to derive some properties of 1-dimensional linear systems with equality in the Plücker estimate. If $\varphi$ is an arbitrary quaternionic holomorphic section, then $N$ with $J \varphi=\varphi N$ is continuous at the zeros of $\varphi$ and smooth elsewhere; cf. the appendix to 3 . The following lemma together with Riemann's removable singularity theorem implies that $N$ is everywhere smooth in the case that $\varphi$ spans a 1 -dimensional linear system with equality in the Plücker estimate.

Lemma 8.13. Let $L$ be a quaternionic holomorphic line bundle with complex structure $J$ over a compact Riemann surface. 
i) Let $\varphi \in H^{0}(L) \backslash\{0\}$ and define $N$ by $J \varphi=\varphi N$. The 1-dimensional linear system spanned by $\varphi$ has equality in the Plücker estimate if and only if $d N^{\prime}=$ $\frac{1}{2}(d N-N * d N) \equiv 0$.

ii) Let $\varphi, \psi \in H^{0}(L)$ and $\psi=\varphi f$ with non-constant $f$ defined away from the zeros of $\varphi$. The 1-dimensional linear system spanned by $\varphi$ has equality in the Plücker estimate if and only if $f$ is Euclidean minimal.

iii) If the Willmore energy of $L$ is non-zero, then $L$ has at most one linear system with equality in the Plücker estimate.

Proof. (i) Equality in the Plücker estimate for the 1-dimensional linear system spanned by $\varphi$ is equivalent to $W\left(L^{-1}, \nabla^{\prime \prime}\right)=\frac{1}{2} \int d N^{\prime} \wedge * d N^{\prime}=0$; see Section 3.6. This is equivalent to $d N^{\prime} \equiv 0$, because $d N^{\prime} \wedge * d N$ is a positive real-valued 2-form. (iii) follows from (i), because $* d f=N d f$ and $d N^{\prime}=d f H$; see Appendix A.4. iiii) Assume $\varphi$ and $\psi \in H^{0}(L)$ span two different 1-dimensional linear systems with equality. Then $f$ and $f^{-1}$ defined by $\psi=\varphi f$ are both Euclidean minimal. This implies that $f$ is planar and, consequently, the Willmore energy of $L$ vanishes; cf. Section 7.3 .

Proof of Theorem 8.11. Let $L=\psi \mathbb{H} \subset \mathbb{H}^{2}, \psi=\left(\begin{array}{l}f \\ 1\end{array}\right)$ be a soliton sphere in the conformal 3-sphere $S^{3}=\operatorname{Im} \mathbb{H} \cup\{\infty\} \subset \mathbb{H} \mathbb{P}^{1}$ with affine representation $f: \mathbb{C P}^{1} \rightarrow$ $\operatorname{Im} \mathbb{H}$. As explained in Section 8.1 the choice $\infty=\left(\begin{array}{l}1 \\ 0\end{array}\right) \mathbb{H}$ makes $L$ into a quaternionic spin bundle for which $\psi \in H^{0}(L)$. Using the Plücker estimate and Theorem 5.3. the assumption $16 \pi \leq W(L) \leq 32 \pi$ implies that the full space of holomorphic sections $H^{0}(L)$ of $L$ is 2-dimensional and has equality in the Plücker estimate. Since $H=H^{0}(L)$, we obtain in particular that the assumption $B\left(H^{*}\right) \subset H$ of Proposition 8.8 is satisfied.

Denote by $\varphi \in H^{0}\left(\mathbb{H}^{2} / L\right)$ the projection of $e_{1}$ to the Möbius invariant holomorphic line bundle $\mathbb{H}^{2} / L$ of $L$. The canonical linear system of $L$ is then spanned by $\varphi, \varphi f$; cf. Section 2.5. Let $\nabla$ be the flat connection of $\mathbb{H}^{2} / L$ such that $\nabla \varphi=0$. Then $\varphi d f$ is a holomorphic section of $K \mathbb{H}^{2} / L$ when $K \mathbb{H}^{2} / L$ is, as in Section 5.2. equipped with the holomorphic structure $d^{\nabla}$. The derivative $\delta: L \rightarrow K \mathbb{H}^{2} / L$ of $L$ is then a complex quaternionic line bundle isomorphism, cf. Section 2.3, which is holomorphic because $\delta$ maps the holomorphic section $\psi \in H^{0}(L)$ to $\varphi d f \in H^{0}\left(K \mathbb{H}^{2} / L\right)$. Using this isomorphism, Lemma 5.5 applied to $\varphi$ implies that $H^{0}\left(\mathbb{H}^{2} / L\right)$ is 3-dimensional and has equality in the Plücker estimate, because $\mathbb{C P}^{1}$ is simply connected.

(a) Suppose that the dual curve $L^{d}$ of $H^{0}(L)$ is of type 国) in Proposition 8.8 i.e., the mean curvature sphere congruence $S$ of $L^{d}$ is everywhere defined, immersed, and skew with respect to a non-degenerate Hermitian form $\langle\cdot, \cdot\rangle$. Since $L^{d}$ is twistor holomorphic one has $\nabla S=2 * Q$. In the proof of Proposition 8.8 it is shown that the isomorphism of $L$ and $K L^{-1}$ is provided by $\nabla S=2 * Q$; i.e., $d f=(\psi, \psi)=$ $2\langle\psi, * Q \psi\rangle$. Thus, without loss of generality,

$$
f=\langle\psi, S \psi\rangle \text {. }
$$

If $\langle\psi, \psi\rangle=0$, then $f^{-1}$ is Euclidean minimal by Corollary B.6, and Lemma 8.13 implies that the 1-dimensional linear system spanned by $\varphi f$ has equality in the Plücker estimate.

If $\langle\psi, \psi\rangle \neq 0$ we may assume that $\langle\psi, \psi\rangle=1$. Let $e_{1}, e_{2}$ be a basis of $H^{0}(L)$ such that $e_{2}=\psi,\left\langle e_{1}, e_{2}\right\rangle=0$, and $L^{d}=\left[\begin{array}{l}g \\ 1\end{array}\right]$ with nowhere-vanishing $g$. From the 
formula for $S$ in Appendix A.4 we then obtain

$$
f=\langle\psi, S \psi\rangle=H_{g} g-R_{g} .
$$

Since $L^{d}$ is twistor holomorphic, $d R_{g}=d R^{\prime}=H_{g} d g$ and hence $d f=d H_{g} g$. By Lemma 6.4 this implies that $f^{\sharp}=f-H_{g} g=-R_{g}$ is a Darboux transform of $f$. This shows that $f$ is a Bryant sphere with smooth ends: because $f^{\sharp}=-R_{g}$ is totally umbilic and both $f$ and $f^{\sharp}=-R_{g}$ take values in $\operatorname{Im}(\mathbb{H})$, we obtain from [19, 5] (see also Remark 6.9) that, away from the isolated points where $f$ and $f^{\sharp}$ intersect, $f$ is a Bryant surface. Since the immersion $f$ is defined on all of $\mathbb{C P}^{1}$, the isolated intersection points are smooth Bryant ends.

Moreover, by Lemma 8.13, the 1 -dimensional linear system of $\mathbb{H}^{2} / L$ that is spanned by $\varphi H_{g}$ has equality in the Plücker estimate, since $H_{g}^{-1}$ is Euclidean minimal by Corollary B.5.

(b) Now suppose that the dual curve $L^{d}$ of $H^{0}(L)$ is of type b) in Proposition 8.8 i.e., there exists a line $\infty \subset H^{0}(L)$ such that $L^{d}$ is Euclidean superminimal in $P H^{0}(L) \backslash\{\infty\}$. Let $e_{1}, e_{2} \in H^{0}(L)$ be a basis such that $e_{1}=\psi$ and $e_{2} \in \infty$ and let $L^{d}=\left[\begin{array}{l}g \\ 1\end{array}\right]$ with respect to this basis. Then $g^{-1}$ is Euclidean superminimal. The description of the spin pairing $K L^{-1} \cong L$ in the proof of Proposition 8.8 implies that $\left(e_{2}, e_{2}\right)=e_{2}^{*}\left(2 * Q e_{2}\right)=d H_{g} g$ and

$$
d f=(\psi, \psi)=\left(e_{2} g^{-1}, e_{2} g^{-1}\right)=\bar{g}^{-1} d H_{g}=-d \bar{H}_{g} g^{-1},
$$

where the last equation holds because $d f$ is $\operatorname{Im}(\mathbb{H})$-valued. Thus $d f g+d \bar{H}_{g}=0$ and Lemma 6.4 implies that $f^{\sharp}=f+\bar{H}_{g} g^{-1}$ is a Darboux transform of $f$. Moreover, $\bar{H}_{g}^{-1}$ is Euclidean minimal by Corollary B.5 and twistor holomorphic, by (iii) of Theorem B.4 because $\bar{H}_{g}$ is a 1-step forward Bäcklund transform of $\bar{g}^{-1}$ which is Euclidean minimal. Thus $f^{\sharp}$ is planar by Lemma 6.5 because both $g^{-1}$ and $\bar{H}_{g}^{-1}$ are Euclidean superminimal. By Lemma 8.13, the 1-dimensional linear system spanned by $\varphi \bar{H}_{g}$ has equality in the Plücker estimate.

To see that $f$ is a Bryant sphere with smooth ends, by [19, 5] it remains to prove that the planar Darboux transform $f^{\sharp}$ is $\operatorname{Im}(\mathbb{H})$-valued (see Remark 6.9): by assumption $e_{2} \mathbb{H}$ is a fixed line of $S$ such that $e_{2}^{*}\left(S e_{2}\right)$ is a quaternion that squares to -1 and is hence imaginary. Because on the other hand $e_{2}^{*}\left(S e_{2}\right)=H_{g} g-R_{g}$ we obtain that $H_{g} g$ takes values in $\operatorname{Im}(\mathbb{H})$. This implies $f^{\sharp}=f+\bar{H}_{g} g^{-1}$ is $\operatorname{Im}(\mathbb{H})$ valued.

Theorem 8.11 yields that the possible Willmore energies of soliton spheres in 3space coincide with the possible Willmore energies of Willmore spheres in 3-space and of Bryant spheres with smooth ends.

Theorem 8.14. The possible Willmore energies $W=\int|\mathcal{H}|^{2}$ of immersed soliton spheres in 3 -space are $W \in 4 \pi(\mathbb{N} \backslash\{0,2,3,5,7\})$.

\section{Concluding Remarks}

Soliton spheres form a class of immersed spheres that are "algebraic" in the sense that they admit rational, conformal parametrizations through a twistorial construction described in terms of quaternionic holomorphic geometry. The class of soliton spheres is large enough to include all known examples of explicitly conformally parametrized immersions of the sphere. It would be interesting to know whether it actually includes all rational, conformal immersions. 
An obvious question is whether there exist further interesting classes of soliton spheres apart from Willmore spheres, Bryant spheres with smooth ends and Taimanov soliton spheres. Potential new examples in $S^{3}$ are presumably more complicated: they would necessarily have Willmore energy $W \geq 36 \pi$ (Theorem 8.11) and possibly higher soliton number (it is not clear to the authors whether Willmore spheres and Bryant spheres with smooth ends are the only 2-soliton spheres in $S^{3}$, i.e., whether there are 2-soliton spheres in $S^{3}$ satisfying $H \cap B\left(H^{*}\right)=\{0\}$ in Remark 8.77.

In this context it would be interesting to establish a description of soliton spheres (at least those with topologically trivial normal bundle) as the immersions stationary under one of the higher flows of the Davey-Stewartson hierarchy. This would provide a link between soliton spheres and the soliton solutions to the DaveyStewartson equations and hence generalize the relation between Taimanov soliton spheres and $\mathrm{mKdV}$-solitons. One challenge in establishing such a link is that, unlike immersions with special symmetry as Taimanov's spheres, general soliton spheres do not (at least not obviously) come with a distinguished conformal vector field (up to scale). Since the Davey-Stewartson hierarchy depends on the choice of such a conformal (= holomorphic) vector field, one would have to deal with a whole $\operatorname{sl}(2, \mathbb{C})$-worth of hierarchies of soliton deformations.

The picture one would expect is that the Davey-Stewartson hierarchy on (a component of) the moduli space of immersed spheres contains the set of soliton spheres as the invariant submanifold on which the orbits of the hierarchy are finite dimensional. Moreover, the order of the flow under which a soliton sphere becomes stationary should essentially correspond to its soliton number. The 0 -soliton is the round sphere, examples of 2 -solitons include Willmore spheres in $S^{3}$ and Bryant spheres with smooth ends, the solutions to certain geometric variational problems (1-solitons are superconformal Willmore spheres and hence not contained in a $3^{-}$ sphere $S^{3}$ ). The investigation of soliton spheres in $S^{3}$ with higher soliton number should lead to new examples. Taimanov soliton spheres have arbitrarily high soliton number and are good candidates for the simplest examples of soliton spheres of a given soliton number.

Parts of this picture persist for immersion of compact Riemann surfaces of higher genus. At least in the case of genus $g=1$, the space of immersions again contains an "algebraic" part which corresponds to special solutions to the Davey-Stewartson equation and contains the solutions to variational problems such as constant mean curvature and constrained Willmore tori; cf. [3]. But the structure of this algebraic part of the moduli space is much richer in this case: apart from a "holomorphic" part of immersions that are essentially parametrized in terms of elliptic functions and, like soliton spheres, have quantized Willmore energy, there is another part of immersions whose explicit parametrizations involve methods of the theory of "finite gap" integration.

\section{Appendix A. Formulae in AfFine Coordinates}

This appendix relates projective invariants of holomorphic curves in $\mathbb{H} \mathbb{P}^{1}$ to the Euclidean invariants of their representations in affine charts.

A.1. Branch points of holomorphic curves in $\mathbb{H} \mathbb{P}^{1}$. A branch point of order $k \in \mathbb{N}$ of a smooth map $f: M \rightarrow N$ from a 2 -dimensional manifold $M$ to an $(n+2)$ dimensional manifold $N$ is a point $p \in M$ for which there exists an integer $k \geq 1$ 
and centered coordinates $z: M \supset U \rightarrow \mathbb{C}$ and $u=\left(u_{1}, \ldots, u_{n+2}\right): N \supset V \rightarrow \mathbb{R}^{n+2}$ at $p$ and $f(p)$ that satisfy

$$
u_{1} \circ f+\mathbf{i} u_{2} \circ f=z^{k+1}+O(k+2), \quad u_{l} \circ f=O(k+2), l=3, \ldots, n+2 ;
$$

cf. [18. We write $b_{p}(f)=k$ for the branching order of $f$ at $p$ and $b(f)$ for the branching divisor of $f$. A map $f$ is called a branched immersion if all points at which $d f$ fails to be injective are branch points. If $M$ is a Riemann surface, a branched immersion is called conformal if it is conformal away from its branch points. The following proposition relates the branching divisor of a holomorphic curve in $\mathbb{H} \mathbb{P}^{1}$ to the Weierstrass divisor of its canonical linear system; cf. Sections 2.5 and 2.6 .

Proposition A.2. A non-constant holomorphic curve in $\mathbb{H} \mathbb{P}^{1}$ is a branched conformal immersion. Its branching divisor coincides with the Weierstrass divisor of its canonical linear system.

Proof. Using the affine chart induced by the basis $e_{1}, e_{2}$ (cf. Section 2.4), a holomorphic curve can be written as $L=\left(\begin{array}{c}f \\ 1\end{array}\right) \mathbb{H} \subset \mathbb{H}^{2}$ with $f: M \rightarrow \mathbb{H}$. The induced basis $e_{1}^{*}, e_{2}^{*} \in H^{0}\left(L^{-1}\right)$ of the canonical linear system then satisfies $e_{1}^{*}=e_{2}^{*} \bar{f}$. Assume that $L_{p}=e_{2} \mathbb{H}$ at $p \in M$ and hence $f(p)=0$. By [13, Section 3.3] there exists a centered holomorphic coordinate $z: M \supset U \rightarrow \mathbb{C}$ at $p$ and a nowhere-vanishing section $\varphi \in \Gamma\left(L_{\mid U}^{-1}\right)$, such that the holomorphic section $e_{1}^{*}=e_{2}^{*} \bar{f}$ of $L^{-1}=\left(\mathbb{H}^{2}\right)^{*} / L^{\perp}$ can be written as

$$
e_{2}^{*} \bar{f}=e_{1}^{*}=z^{k+1} \varphi+O(k+2) \quad \text { as } z \rightarrow \infty .
$$

This proves the claim, because $b_{p}(f)=k=\operatorname{ord}_{p}\left(e_{1}^{*}\right)-1$ and $\operatorname{ord}_{p}\left(\mathbb{H}^{2}\right)^{*}=\operatorname{ord}_{p}\left(e_{1}^{*}\right)-$ $1=k$ as $e_{2}^{*}$ induces a section of $L^{-1}$ which does not vanish at $p$.

A.3. Left and right normal vectors. Let $L=\left(\begin{array}{c}f \\ 1\end{array}\right) \mathbb{H} \subset \mathbb{H}^{2}$ be a smooth map into $\mathbb{H}^{1}$. Then $f: M \rightarrow \mathbb{H}$ is the image of $L$ under the affine chart $\sigma$ defined by $e_{1}, e_{2}$. As explained in Section 2.4 the map $L$ is a holomorphic curve if and only if there exists a smooth map $R: M \rightarrow S^{2} \subset \operatorname{Im} \mathbb{H}$ such that

$$
* d f=-d f R \text {. }
$$

Analogously, the dual map $L^{\perp}$ is a holomorphic curve if and only if there exists a smooth map $N: M \rightarrow S^{2} \subset \operatorname{Im} \mathbb{H}$ such that

$$
* d f=N d f
$$

because $L^{\perp}=\left(e_{1}^{*}-e_{2}^{*} \bar{f}\right) \mathbb{H} \subset\left(\mathbb{H}^{2}\right)^{*}$. The maps $N$ and $R$ are called the left normal and the right normal of $f$, respectively. If $f$ is a conformal immersion, both normal vectors exist and are smooth. One can prove (cf. appendix to [3] ) that if $f$ is a non-constant map for which one of the two normals exists and is smooth, the other normal vector can be globally defined as a continuous map which is smooth away from the branch points of $f$.

Recall from Section 2.5 that if $L$ is a holomorphic curve, then $L^{-1}=\left(\mathbb{H}^{2}\right)^{*} / L^{\perp}$ is a Möbius invariant holomorphic line bundle. The constant sections of $\left(\mathbb{H}^{2}\right)^{*}$ project to the canonical linear system of holomorphic sections which is spanned by $e_{1}^{*}=e_{2}^{*} \bar{f}$ and $e_{2}^{*}$. In particular, the complex structure $J$ of $L^{-1}$ satisfies $J e_{2}^{*}=e_{2}^{*} R$. Similarly, if $L^{\perp}$ is holomorphic, then $\mathbb{H}^{2} / L=\left(L^{\perp}\right)^{-1}$ is a Möbius invariant holomorphic line bundle whose canonical linear system is spanned by $e_{1}$ and $e_{2}=-e_{1} f$ and whose complex structure $J$ satisfies $J e_{1}=e_{1} N$. 
A.4. Mean curvature sphere congruence. Assume now that $L$ is immersed. Then both Möbius invariant quaternionic holomorphic line bundles are defined. The left and right normals $N$ and $R$ are both smooth and $* d f=N d f=-d f R$. In [10, Section 7.1] it is shown that the mean curvature vector $\mathcal{H}$ of $f$ is related to $N$ and $R$ via

$$
d N^{\prime}=\frac{1}{2}(d N-N * d N)=d f H \quad \text { and } \quad d R^{\prime}=\frac{1}{2}(d R-R * d R)=H d f,
$$

where $H=-R \overline{\mathcal{H}}=-\overline{\mathcal{H}} N$. In particular $f$ is Euclidean minimal if and only if $N$ and $R$ are anti-holomorphic, i.e., $d N^{\prime}=d R^{\prime}=0$.

The mean curvature sphere congruence of $L$ (Section 7.1) is given by

$$
S=\operatorname{Ad}\left(\begin{array}{ll}
1 & f \\
0 & 1
\end{array}\right)\left(\begin{array}{cc}
N & 0 \\
-H & -R
\end{array}\right)=\left(\begin{array}{cc}
N-f H & f H f-N f-f R \\
-H & H f-R
\end{array}\right)
$$

(see [10, Section 7.2]) and its Hopf fields are

$$
2 * A=\operatorname{Ad}\left(\begin{array}{cc}
1 & f \\
0 & 1
\end{array}\right)\left(\begin{array}{cc}
0 & 0 \\
w & d R^{\prime \prime}
\end{array}\right) \quad \text { and } \quad 2 * Q=\operatorname{Ad}\left(\begin{array}{ll}
1 & f \\
0 & 1
\end{array}\right)\left(\begin{array}{cc}
d N^{\prime \prime} & 0 \\
w-d H & 0
\end{array}\right),
$$

with $d R^{\prime \prime}=\frac{1}{2}(d R+R * d R), d N^{\prime \prime}=\frac{1}{2}(d N+N * d N)$, and

$$
w=\frac{1}{2}\left(d H+R * d H-H * d N^{\prime \prime}\right)=\frac{1}{2}\left(d H+* d H N-* d R^{\prime \prime} H\right) .
$$

The mean curvature sphere congruence of the dual curve $L^{\perp}$ is $S^{\perp}=S^{*}$ with Hopf fields $A^{\perp}=-Q^{*}$ and $Q^{\perp}=-A^{*}$.

By Lemma 3.8, the holomorphic curve $L$ is twistor holomorphic if and only if $d R^{\prime \prime}=0$. Similarly $L^{\perp}$ is twistor holomorphic if and only if $d N^{\prime \prime}=0$. Moreover, $L$ is totally umbilic if and only if $S$ is constant, which is equivalent to both $L$ and $L^{\perp}$ being twistor holomorphic., i.e., to $d N^{\prime \prime}=d R^{\prime \prime}=0$.

\section{Appendix B. BäCKLUND transformations of Willmore SURFACES}

In this appendix we collect some results from [10, Section 9] about 1- and 2-step Bäcklund transformations of Willmore surfaces in the conformal 4 -sphere $S^{4}=\mathbb{H} \mathbb{P}^{1}$ and derive consequences that are central for the present paper. As an example we show how the Weierstrass representation of minimal surfaces fits into this context.

B.1. 2-step Bäcklund transformation. A holomorphic curve $L \subset \mathbb{H}^{2}$ in $\mathbb{H}^{1}$ is Willmore if and only if its Hopf fields $A$ and $Q$ are co-closed; see Section 7.1. If $A$ does not vanish identically, then $\vec{L}=\operatorname{ker}(A)$ extends smoothly through the isolated zeros of $A$ to either a branched conformal immersion or a constant map. If $Q$ does not vanish identically, the same is true for $\overleftarrow{L}=\operatorname{im}(Q)$; cf. [10, Appendix 13.1]. The maps $\vec{L}$ and $\overleftarrow{L}$ defined by

$$
\vec{L} \supset \operatorname{ker}(A) \quad \text { and } \quad \overleftarrow{L} \subset \operatorname{im}(Q)
$$

are then called the 2-step forward or backward Bäcklund transform of $L$, respectively. If $\vec{L}$ is defined and non-constant, by [10, Theorem 7] its Hopf field is $\vec{Q}=A$, which implies that $\vec{L}$ is again Willmore and $\overleftrightarrow{\vec{L}}=L$. Analogously, $\overleftarrow{A}=Q$ and $\stackrel{\vec{L}}{L}=L$. 
B.2. 1-step Bäcklund transformation. In contrast to 2-step Bäcklund transformations, 1-step Bäcklund transformations are not Möbius invariant but depend on the choice of a point $\infty \in \mathbb{H P}^{1}$ : as in Appendix $\mathrm{A}$ we chose $\infty=\left[\begin{array}{l}1 \\ 0\end{array}\right]$ and write $L=\left(\begin{array}{c}f \\ 1\end{array}\right) \mathbb{H} \subset \mathbb{H}^{2}$ with $f: M \rightarrow \mathbb{H}$ the representation in the affine chart defined by $e_{1}, e_{2}$. Because $w$ in Appendix A.4 satisfies $* w=-R w$ and $*(w-d H)=(w-d H) N$ we have

$$
d f \wedge w=(w-d H) \wedge d f=0 .
$$

One can check [10, Section 7] that $w$ is closed if and only if $A$ or, equivalently, $Q$ is co-closed, which in turn is equivalent to $f$ being Willmore (see Section 7).

If $f$ is a Willmore immersion, a smooth map $g: M \rightarrow \mathbb{H}$ that satisfies

$$
d g=w=e_{2}^{*}\left(2 * A e_{1}\right)
$$

is called a 1-step forward Bäcklund transform of $f$. Up to similarity transformation $g$ is uniquely determined by the choice of $\infty$. If $w$ does not vanish identically, then $g$ is a branched conformal immersion. Similarly, a map $h$ with $d h=w-d H=$ $e_{2}^{*}\left(2 * Q e_{1}\right)$ is called a $1-$ step backward Bäcklund transform.

B.3. Properties of the Bäcklund transformations. The following theorem describes the relation between 1-step and 2-step Bäcklund transformations.

Theorem B.4. Let $f: M \rightarrow \mathbb{H}$ be a Willmore immersion and $g: M \rightarrow \mathbb{H}$ a 1-step forward Bäcklund transform, i.e., $d g=w$. If $w \neq \equiv$, then:

i) The 1-step forward Bäcklund transform $g$ is a branched conformal Willmore immersion. Away from its branch points $d f=w_{g}-d H_{g}$. In particular, $f$ is a 1-step backward Bäcklund transform of $g$ and $f+H_{g}$ is a 1-step forward Bäcklund transform of $g$.

ii) The 2-step forward Bäcklund transform $\vec{f}$ of $f$ coincides with the 1-step forward Bäcklund transform $\vec{f}=f+H_{g}$ of $g$.

iii) The 1-step forward Bäcklund transform $g$ of $f$ is twistor holomorphic, i.e., $A_{g} \equiv 0$, if and only if there exists a point $a \in \mathbb{H}$ such that the inversion $(f-a)^{-1}$ is Euclidean minimal.

The analogous statements hold for backward Bäcklund transformations.

Proof. Statement il) is an immediate consequence of the fact [10, Proposition 16] that if two branched conformal immersions satisfy $d f \wedge d g=0$, then $d g\left(w_{g}-d H_{g}\right)=$ $w d f$. Statement (ii) follows from [10, Lemma 10]. To see iii), note that since $A \not \equiv 0$ the characterization of Euclidean minimal surfaces given in Section 7.3 yields that $(f-a)^{-1}$ is Euclidean minimal if and only if $\left[\begin{array}{l}a \\ 1\end{array}\right] \subset \operatorname{ker} A$. But this means that $\left[\begin{array}{l}a \\ 1\end{array}\right]$ is the 2-step forward Bäcklund transform of $f$ which, by ii) and (ii), is equivalent to $w_{g} \equiv 0$. Because $w_{g} \equiv 0$, the assumption $A_{g} \not \equiv 0$ would imply that $g$ is Euclidean minimal, i.e., $H_{g} \equiv 0$, which contradicts $a=\vec{f}=f+H_{g}$. Thus, for the 1 -step Bäcklund transformation $g$ we have that $w_{g} \equiv 0$ is equivalent to $A_{g} \equiv 0$.

Using the formula for $S$ in Section A.4 we obtain:

Corollary B.5. Let $f: M \rightarrow \mathbb{H}$ be a nowhere-vanishing conformal immersion of a simply connected Riemann surface. Its inversion $f^{-1}$ is Euclidean minimal if and only if there is a branched twistor holomorphic immersion $g$ with mean curvature 
sphere congruence $S_{g}$ such that

$$
f=-H_{g}=e_{2}^{*}\left(S_{g} e_{1}\right)
$$

away from the branch points of $g$.

In other words, $f^{-1}$ is Euclidean minimal if and only if $\bar{f}=-N_{g} \mathcal{H}_{g}=-\mathcal{H}_{g} R_{g}$; i.e., $\bar{f}$ is the rotation of the mean curvature vector $\mathcal{H}_{g}$ of a twistor holomorphic curve $g$ by minus $\pi / 2$ in the normal bundle of $g$. Note that locally every branched twistor holomorphic immersion $g$ into $\mathbb{H}$ that is neither totally umbilic nor Euclidean minimal is a 1-step forward Bäcklund transformation of $f$ as in the preceding corollary.

By Richter's theorem [10, Theorem 9], a Willmore surface $f: M \rightarrow \mathbb{H}$ that is not Euclidean minimal takes values in $\mathbb{R}^{3}=\operatorname{Im} \mathbb{H}$ if and only if it has a 1 -step forward Bäcklund transform $g$ that is minimal with respect to the hyperbolic geometry defined by the Hermitian form

$$
\left\langle\left(\begin{array}{l}
x_{1} \\
x_{2}
\end{array}\right),\left(\begin{array}{l}
y_{1} \\
y_{2}
\end{array}\right)\right\rangle=\bar{x}_{2} y_{1}+\bar{x}_{1} y_{2} .
$$

(By Lemma C.10 the latter is equivalent to $S_{g}$ being skew with respect to $\langle\cdot, \cdot\rangle$. The hyperbolic minimal Bäcklund transforms are then characterized by the property that $\operatorname{Re}(g)=\frac{1}{2} H$.) We obtain:

Corollary B.6. Let $f: M \rightarrow \mathbb{R}^{3}=\operatorname{Im} \mathbb{H}$ be a nowhere-vanishing conformal immersion of a simply connected Riemann surface. Its inversion $f^{-1}$ is Euclidean minimal if and only if there is a branched twistor holomorphic immersion $g$ with mean curvature sphere congruence $S_{g}$ that is hyperbolic minimal with respect to $\langle\cdot, \cdot\rangle$ and a null vector $e_{1} \in \mathbb{H}^{2}$ such that

$$
f=-H_{g}=\left\langle e_{1}, S_{g} e_{1}\right\rangle
$$

away from the branch points of $g$.

In other words, $f^{-1}$ is Euclidean minimal if and only if $f=N_{g} \mathcal{H}_{g}=\mathcal{H}_{g} R_{g}$; i.e., $f$ is the rotation of the mean curvature vector $\mathcal{H}_{g}$ of a twistor holomorphic curve $g$ by $\pi / 2$ in the normal bundle of $g$.

B.7. Weierstrass representation and 1-step Bäcklund transformation of twistor holomorphic curves. The formula $f=-H_{g}=e_{2}^{*}\left(S e_{1}\right)$ in Corollary B.5 can be seen as an integral free version of the Weierstrass representation of the minimal surface $f^{-1}$ : we will show that $f^{-1}=\left(e_{2}^{*}\left(S e_{1}\right)\right)^{-1}$ is the imaginary part of a holomorphic null curve in $\mathbb{C}^{4}$ obtained from $g$.

Right multiplication by i makes $\mathbb{H}^{2}$ into a complex 4-dimensional vector space $\mathbb{C}^{4} \cong\left(\mathbb{H}^{2}, \mathbf{i}\right)$. Let $\left[\begin{array}{l}g \\ 1\end{array}\right] \subset \mathbb{H}^{2}$ be a twistor holomorphic curve as in Corollary B.5 (i.e., $g$ is neither Euclidean minimal nor totally umbilic). Away from the branch points of $\left[\begin{array}{l}g \\ 1\end{array}\right]$, the $\mathbf{i}$-eigenspaces of its mean curvature sphere congruence $S$ coincide with the tangent line congruence (or first osculating curve) of its twistor lift; cf. Appendix C. Because by assumption $H_{g}$ is an immersion, the vector $e_{1}$ is only at isolated points an eigenvector of $S$. The tangent line congruence of the twistor holomorphic curve $\left[\begin{array}{l}g \\ 1\end{array}\right]$ is, away from these isolated points, the holomorphic null immersion

$$
[\hat{S}]: M \rightarrow Q^{4}, \quad \hat{S}=\left(e_{1}-S e_{1} \mathbf{i}\right) \wedge\left(e_{1}+S e_{1} \mathbf{i}\right) \mathbf{j}
$$


in $Q^{4}=\left\{[v] \in P\left(\Lambda^{2}\left(\mathbb{H}^{2}, \mathbf{i}\right)\right) \mid v \wedge v=0\right\}$, where $e_{1}, e_{2}$ is the standard basis of $\mathbb{H}^{2}$ and where null means that the tangent lines of $[\hat{S}]$ are contained in $Q^{4}$. Consider the real structure on $\Lambda^{2}\left(\mathbb{H}^{2}, \mathbf{i}\right)$ defined by $x \wedge y \mapsto x \mathbf{j} \wedge y \mathbf{j}$. A real basis of $\Lambda^{2}\left(\mathbb{H}^{2}, \mathbf{i}\right)$ is then given by

$$
\begin{aligned}
& \hat{e}_{1}=e_{1} \wedge e_{2} \mathbf{j}-e_{1} \mathbf{j} \wedge e_{2}, \quad \hat{e}_{2}=\left(e_{1} \wedge e_{2} \mathbf{j}+e_{1} \mathbf{j} \wedge e_{2}\right) \mathbf{i}, \\
& \hat{e}_{3}=e_{1} \wedge e_{2}+e_{1} \mathbf{j} \wedge e_{2} \mathbf{j}, \quad \hat{e}_{4}=\left(e_{1} \wedge e_{2}-e_{1} \mathbf{j} \wedge e_{2} \mathbf{j}\right) \mathbf{i}, \\
& \hat{e}_{5}=e_{1} \wedge e_{1} \mathbf{j}, \quad \hat{e}_{6}=e_{2} \wedge e_{2} \mathbf{j} .
\end{aligned}
$$

A complex bilinear symmetric form $\langle\cdot, \cdot\rangle$ on $\Lambda^{2}\left(\mathbb{H}^{2}, \mathbf{i}\right)$ can be defined by $\langle x, y\rangle \hat{e}_{5} \wedge$ $\hat{e}_{6}=x \wedge y$. The affine part of the stereographic projection with "pole" $\left[e_{5}\right]$ onto the tangent plane to $Q^{4}$ at $\left[e_{6}\right]$ is then

$$
\hat{\sigma}(\hat{S})=\left(\hat{S}-\left\langle\hat{S}, \hat{e}_{6}\right\rangle \hat{e}_{5}\right)\left\langle\hat{S}, \hat{e}_{5}\right\rangle^{-1}-\hat{e}_{6},
$$

which is a meromorphic null curve in $\mathbb{C}^{4} \cong \operatorname{Span}\left\{\hat{e}_{1}, \hat{e}_{2}, \hat{e}_{3}, \hat{e}_{4}\right\}$. Writing $S e_{1}=$ $e_{1} h_{1}+e_{2} h_{2}$ we obtain

$$
\left\langle\hat{S}, \hat{e}_{5}\right\rangle=-\left|h_{2}\right|^{2} \quad \text { and } \quad \operatorname{Im}\left(\hat{S}-\left\langle\hat{S}, \hat{e}_{6}\right\rangle \hat{e}_{5}-\left\langle\hat{S}, \hat{e}_{5}\right\rangle \hat{e}_{6}\right)=-\bar{h}_{2},
$$

where we identify $\operatorname{Im}\left(\mathbb{C}^{4}\right) \cong \mathbb{H}$ via $\hat{e}_{1} \mathbf{i} \mapsto 1, \hat{e}_{2} \mathbf{i} \mapsto \mathbf{i}, \hat{e}_{3} \mathbf{i} \mapsto \mathbf{j}, \hat{e}_{4} \mathbf{i} \mapsto \mathbf{k}$. Hence

$$
\operatorname{Im}(\hat{\sigma}(\hat{S}))=\bar{h}_{2}\left|h_{2}\right|^{-2}=h_{2}^{-1}=\left(e_{2}^{*}\left(S e_{1}\right)\right)^{-1}=f^{-1} .
$$

The minimal surface $f^{-1}$ and hence $f$ is contained in $\operatorname{Im} \mathbb{H}=\mathbb{R}^{3}$ if and only if $\hat{S}$ is orthogonal to $\hat{e}_{1}$ in $\Lambda^{2}\left(\mathbb{H}^{2}, \mathbf{i}\right)$ with respect to $\langle\cdot, \cdot\rangle$. But this can be shown to be equivalent to $S$ being skew Hermitian with respect to the Hermitian form $\langle\cdot, \cdot\rangle$ in Corollary B.6 which, by Lemma C.10, is again equivalent to $g$ being hyperbolic superminimal.

\section{Appendix C. Twistor holomorphic CURVEs In $\mathbb{H}^{1}$}

This appendix relates properties of the mean curvature sphere congruence $S$ of a twistor holomorphic curve to properties of the osculating curves of its twistor lift.

A twistor holomorphic curve in $\mathbb{H}^{1}$ is a holomorphic curve whose Hopf field $A$ vanishes identically (Lemma 3.8) and which hence is in particular Willmore (cf. Section 7.1). Equivalently, a holomorphic curve in $\mathbb{H}^{1}$ is twistor holomorphic if and only if the derivative $\nabla S$ of its mean curvature sphere congruence $S$ satisfies

$$
\nabla S=2 * Q
$$

C.1. Mean curvature sphere congruence. The $k$-th osculating curve $\hat{L}_{k}$ of a holomorphic curve $\hat{L} \subset \mathbb{C}^{n+1}$ is the analytic continuation of the rank $k+1$ subbundle spanned by the $k$-th derivatives of sections of $\hat{L}$; cf. [17, p. 262]. The first osculating curve or tangent line congruence $\hat{L}_{1}$ of the twistor lift $\hat{L}=\{\psi \in L \mid$ $J \psi=\psi \mathbf{i}\} \subset\left(\mathbb{H}^{2}, \mathbf{i}\right)$ of a twistor holomorphic curve $L$ in $\mathbb{H} \mathbb{P}^{1}$ is the $\mathbf{i}-$ eigenspace of the mean curvature sphere congruence $S$ of $L$, because $S \nabla \varphi=(\nabla S) \varphi \mathbf{i}+\nabla \varphi \mathbf{i}=\nabla \varphi \mathbf{i}$ for all $\varphi \in \Gamma(\hat{L})$. Although $S$ is not defined at branch points of $L$, the tangent line congruence $\hat{L}_{1}$ is, because all osculating curves of complex holomorphic curves extend through their Weierstrass points. We obtain the following characterization of the branch points of $L$ through which the mean curvature sphere congruence extends smoothly. 
Lemma C.2. The mean curvature sphere congruence of a twistor holomorphic curve $L$ extends smoothly through a branch point $p$ of $L$ if and only if at $p$ the tangent line congruence $\hat{L}_{1}$ of its twistor lift is not tangent to the fiber of the twistor projection, i.e., if $\mathbb{H}^{2}=\hat{L}_{\left.1\right|_{p}} \oplus \hat{L}_{1} \mathbf{j}_{\mid p}$.

C.3. 2-step Bäcklund transformation. The complex dual curve of the twistor lift of a twistor holomorphic curve $L$ in $\mathbb{H} \mathbb{P}^{1}$ can again be projected to $\mathbb{H} \mathbb{P}^{1}$. We prove now that the curve thus obtained is the dual curve of the 2-step backward Bäcklund transform $\overleftarrow{L}$ of $L$ (Appendix B.1).

Lemma C.4. Let $L \subset \mathbb{H}^{2}$ be a non-totally umbilic twistor holomorphic curve in $\mathbb{H} \mathbb{P}^{1}$ with twistor lift $\hat{L}$. Its 2 -step backward Bäcklund transform $\overleftarrow{L}=\operatorname{im}(Q)$ extends smoothly through zeros of $Q$ and branch points of $L$ (where the mean curvature sphere $S$ of $L$ and hence $Q$ is not defined) and the dual $\overleftarrow{L}^{\perp} \subset\left(\mathbb{H}^{2}\right)^{*}$ is a twistor holomorphic curve with mean curvature sphere congruence $-S^{*}$ whose twistor lift is the complex dual curve $\hat{L}^{d}$ of $\hat{L}$.

Proof. Since $L$ is twistor holomorphic we have $\nabla S=2 * Q$, and $\hat{L}_{1} \oplus \hat{L}_{1} \mathbf{j}=\mathbb{H}$ away from the isolated branch points of $L$, by Lemma C.2. It suffices to show that $\operatorname{im}(Q) \subset \hat{L}_{2}$, because $\hat{L}^{d}=\hat{L}_{2}^{\perp} \subset\left(\mathbb{H}^{2}, \mathbf{i}\right)^{*}=\left(\mathbb{C}^{4}\right)^{*}$. Let $\varphi \in \Gamma\left(\hat{L}_{1}\right)$ and $\omega_{1}, \omega_{2} \in \Omega^{1}\left(\hat{L}_{1}\right)$ such that $\nabla \varphi=\omega_{1}+\omega_{2} \mathbf{j}$. Then

$$
\begin{aligned}
2 * Q \varphi & =\nabla S \varphi=\nabla(S \varphi)-S \nabla \varphi=\nabla \varphi \mathbf{i}-S \nabla \varphi \\
& =\omega_{1} \mathbf{i}+\omega_{2} \mathbf{j} \mathbf{i}-\omega_{1} \mathbf{i}-\omega_{2} \mathbf{i j}=2 \omega_{2} \mathbf{j} \mathbf{i} .
\end{aligned}
$$

Thus $\operatorname{im}(Q) \subset \hat{L}_{2}$, because $Q \varphi=* \omega_{2} \mathbf{i j}=* \omega_{1} \mathbf{i}-* \nabla \varphi \mathbf{i} \in \Omega^{1}\left(\hat{L}_{2}\right)$ and $Q \varphi \mathbf{j}=$ $-* \omega_{2} \mathbf{i} \in \Omega^{1}\left(\hat{L}_{2}\right)$ and $Q \varphi \mathbf{j} \in \Omega^{1}\left(\hat{L}_{1}\right)$.

C.5. Euclidean minimal twistor holomorphic curves. Using the characterization of Euclidean minimal curves in Section 7.3 we obtain the following characterization of holomorphic curves in $\mathbb{H} \mathbb{P}^{1}$ that are twistor holomorphic and Euclidean minimal.

Lemma C.6. Let $L \subset \mathbb{H}^{2}$ be a twistor holomorphic curve in $\mathbb{H} \mathbb{P}^{1}$ that is not totally umbilic. Then $L$ is Euclidean minimal in $\mathbb{H}=\mathbb{H} \mathbb{P}^{1} \backslash\{\infty\}$ for some $\infty \in \mathbb{H} \mathbb{P}^{1}$ if and only if $\overleftarrow{L}=\operatorname{im}(Q)$ is constant if and only if the twistor lift of $\hat{L} \subset\left(\mathbb{H}^{2}, \mathbf{i}\right)$ lies in a complex plane. The point $\infty \in \mathbb{H} \mathbb{P}^{1}$ then corresponds to the unique $\mathbf{j}$-invariant line in the plane containing $\hat{L}$.

Proof. If $\overleftarrow{L}$ is constant, then $\infty=\overleftarrow{L}$ is a point contained in all mean curvature spheres (because $S$ anti-commutes with $Q$ ) and $L$ is Euclidean minimal in $\mathbb{H} \mathbb{P}^{1} \backslash\{\infty\}$. The point $\infty$ is contained in all mean curvature spheres if and only if the corresponding complex line in $P\left(\mathbb{H}^{2}, \mathbf{i}\right) \cong \mathbb{C P}^{3}$ intersects all tangent lines $\hat{L}_{1}$ of $\hat{L}$. But this implies that $\hat{L}$ is planar (a space curve admitting a line that intersects all its tangents is always planar). If $\hat{L}$ is planar, then $\hat{L}^{d}$ is constant and Lemma C.4 implies that $\overleftarrow{L}$ is constant.

C.7. Branching divisors of the osculating curves of the twistor lift. Let $L$ be a twistor holomorphic curve with twistor lift of $\hat{L}$. The following lemma relates the branching divisors of the osculating curves of $\hat{L}$ to the zero divisor ord $(Q)$ of the 
Hopf field $Q$ of $L$ and the branching divisors $b(L)$ and $b(\overleftarrow{L})$ of $L$ and its backward Bäcklund transform $\overleftarrow{L}$ (extended to the whole Riemann surface as in Lemma C.4).

Lemma C.8. Let $L \subset \mathbb{H}^{2}$ be a twistor holomorphic curve in $\mathbb{H} \mathbb{P}^{1}$ with holomorphic twistor lift $\hat{L} \subset\left(\mathbb{H}^{2}, \mathbf{i}\right)$ for which globally $\mathbb{H}^{2}=\hat{L}_{1} \oplus \hat{L}_{1} \mathbf{j}$. Then

$$
b(\hat{L})=b(L), \quad b\left(\hat{L}_{1}\right)=\operatorname{ord}(Q), \quad b\left(\hat{L}_{2}\right)=b(\overleftarrow{L}) .
$$

The last equality holds if $\hat{L}_{2}$ and hence $\overleftarrow{L}$ is non-constant.

Proof. Clearly $b_{p}(\hat{L}) \leq b_{p}(L)$ for all $p \in M$ with equality if $\hat{L}_{1 \mid p}$ is not a quaternionic subspace. Thus $\hat{L}_{1} \cap \hat{L}_{1} \mathbf{j}=\{0\}$ implies $b(\hat{L})=b(L)$. The same argument applied to $(\overleftarrow{L})^{\perp}$ shows $b\left(\hat{L}_{2}\right)=b(\overleftarrow{L})$, because the complex dual $\hat{L}^{d}$ of $\hat{L}$ is the twistor lift of $(\overleftarrow{L})^{\perp}$, by Lemma C.4 and $b\left(\hat{L}_{2}\right)=b\left(\hat{L}^{d}\right)$. The displayed formula in the proof of Lemma C.4 and $\mathbb{H}^{2}=\hat{L}_{1} \oplus \hat{L}_{1} \mathbf{j}$ yield $b_{p}\left(\hat{L}_{1}\right)=\min \left\{\operatorname{ord}_{p}(\nabla \varphi \mathbf{i}-S \nabla \varphi) \mid \varphi \in\right.$ $\left.\Gamma\left(\hat{L}_{1}\right)\right\}=\min \left\{\operatorname{ord}_{p}(Q \varphi) \mid \varphi \in \Gamma\left(\hat{L}_{1}\right)\right\}=\operatorname{ord}_{p}(Q)$.

C.9. Superminimal curves in $\mathbb{H}^{1}$. A superminimal curve is a twistor holomorphic curve that is minimal with respect to some 4-dimensional space form subgeometry [6, 14, 15, 16.

As in [10, 20] we use quaternionic Hermitian forms in order to describe the space form subgeometries of 4-dimensional Möbius geometry in the quaternionic projective framework: let $\langle\cdot, \cdot\rangle$ be a non-trivial quaternionic Hermitian form on $\mathbb{H}^{2}$ and denote by $I=\left\{[v] \in \mathbb{H}^{1} \mid\langle v, v\rangle=0\right\}$ its set of null lines. Depending on whether $\langle\cdot, \cdot\rangle$ is definite, indefinite, or degenerate, the set $I$ is empty, a round 3 -sphere in $\mathbb{H} \mathbb{P}^{1}$, or a point.

If $\langle\cdot, \cdot\rangle$ is non-degenerate one can define the Riemannian metric

$$
g_{[x]}(v, w):=\frac{4}{\langle x, x\rangle^{2}} \operatorname{Re}(\langle v(x), w(x)\rangle\langle x, x\rangle-\langle v(x), x\rangle\langle x, w(x)\rangle)
$$

on $\mathbb{H} \mathbb{P}^{1} \backslash I$, where $x \in \mathbb{H}^{2}$ and $v, w \in T_{[x]} \mathbb{H} \mathbb{P}^{1}=\operatorname{Hom}\left([x], \mathbb{H}^{2} /[x]\right)$. The Riemannian metric is compatible with the conformal structure on $\mathbb{H}^{1}$, and the Riemannian manifold $\left(\mathbb{H} \mathbb{P}^{1} / I, \pm g\right)$ is isometric to either $S^{4}$ or two copies of hyperbolic 4-space depending on the signature of $\langle\cdot, \cdot\rangle$.

If $\langle\cdot, \cdot\rangle$ is degenerate, the affine chart $\mathbb{H} \mathbb{P}^{1} \backslash\{\infty\} \rightarrow \mathbb{H}$ of Section 2.4 induces, uniquely up to scale, a Euclidean structure on $\mathbb{H}^{1} \backslash I \cong S^{4} \backslash\{\infty\}$.

Lemma C.10. A holomorphic curve in $\mathbb{H}_{\mathbb{P}^{1}}$ is minimal with respect to the space form geometry defined by a Hermitian form $\langle\cdot, \cdot\rangle$ if and only if its mean curvature sphere congruence is skew Hermitian with respect to $\langle\cdot, \cdot\rangle$.

Proof. A surface in a space form is minimal if and only if all its mean curvature spheres are totally geodesic. The 2 -sphere described by the eigenlines of an endomorphism $S$ of $\mathbb{H}^{2}$ that squares to -1 is totally geodesic if and only if the corresponding Möbius transformation is an isometry of the space form defined by $\langle\cdot, \cdot\rangle$ which is equivalent to $S$ being skew Hermitian.

We call a twistor holomorphic curve in $\mathbb{H}^{1}$ all of whose mean curvature spheres are skew with respect to some Hermitian form on $\mathbb{H}^{2}$ spherical, hyperbolic, or Euclidean superminimal depending on the type of the Hermitian form. Note that 
compact superminimal curves in $\mathbb{H}^{1}$ exist in all three cases, although in the hyperbolic and Euclidean cases they go through infinity $I$.

The $(\mathbf{j} \mathbb{C})$-part $\mathbf{j} \Omega=\frac{1}{2}(\langle\cdot, \cdot\rangle+\mathbf{i}\langle\cdot, \cdot\rangle \mathbf{i})$ of the Hermitian form $\langle\cdot, \cdot\rangle$ defines an alternating complex 2 -form $\Omega$ on $\left(\mathbb{H}^{2}, \mathbf{i}\right) \cong \mathbb{C}^{4}$. Push-forward with multiplication by the quaternion $\mathbf{j}$ induces real structures on the complex vector spaces $\Lambda^{2}\left(\mathbb{H}^{2}, \mathbf{i}\right) \cong \mathbb{C}^{6}$ and $\Lambda^{4}\left(\mathbb{H}^{2}, \mathbf{i}\right) \cong \mathbb{C}$. One can check that, by fixing an element in the real part of $\Lambda^{4}\left(\mathbb{H}^{2}, \mathbf{i}\right)$, the wedge product defines a Minkowski product on $\operatorname{Re}\left(\Lambda^{2}\left(\mathbb{H}^{2}, \mathbf{i}\right)\right) \cong \mathbb{R}^{6}$.

Because $\Omega$ is a real linear form on $\Lambda^{2}\left(\mathbb{H}^{2}, \mathbf{i}\right)$ it can be realized as the scalar product with a real element $\Omega^{\sharp} \in \operatorname{Re}\left(\Lambda^{2}\left(\mathbb{H}^{2}, \mathbf{i}\right)\right)$. One can check that $\Omega^{\sharp}$ is time like, space like, or light like depending on whether the Hermitian form is definite, indefinite, or degenerate. Moreover, it can be proven that a twistor holomorphic curve $L$ in $\mathbb{H}^{1}$ is superminimal with respect to the Hermitian form $\langle\cdot, \cdot\rangle$ on $\mathbb{H}^{2}$ if and only if the tangent line congruence $\hat{L}_{1}$ of its twistor lift $\hat{L}$ is polar to $\Omega^{\sharp}$.

Proposition C.11. A holomorphic curve $L \subset \mathbb{H}^{2}$ is spherical, hyperbolic, or Euclidean superminimal if and only if its twistor lift $\hat{L} \subset\left(\mathbb{H}^{2}, \mathbf{i}\right)$ is holomorphic and its tangent line congruence $\hat{L}_{1} \subset \Lambda^{2}\left(\mathbb{H}^{2}, \mathbf{i}\right)$ is polar to a time like, space like, or light like vector in $\operatorname{Re}\left(\Lambda^{2}\left(\mathbb{H}^{2}, \mathbf{i}\right)\right)$.

Remark C.12. The 2 -form $\Omega$ corresponding to $\Omega^{\sharp} \in \operatorname{Re}\left(\Lambda^{2}\left(\mathbb{H}^{2}, \mathbf{i}\right)\right)$ with $\Omega^{\sharp} \wedge \Omega^{\sharp} \neq 0$ induces an isomorphism between the complex vector space $\left(\mathbb{H}^{2}, \mathbf{i}\right)$ and its dual. The tangent line congruence $\hat{L}_{1}$ of $\hat{L}$ is polar to $\Omega^{\sharp}$ if and only if this isomorphism maps $\hat{L}$ to its complex dual $\hat{L}^{d}$ : take a local holomorphic section $\psi$ of $\hat{L}$. Then $\hat{L}_{1}$ is polar to $\Omega^{\sharp}$ if and only if $\Omega\left(\psi, \psi^{\prime}\right)=0$. But this implies that $\Omega\left(\psi, \psi^{\prime \prime}\right)=0$ and is hence equivalent to $\operatorname{ker}(\Omega(\psi,))=.\operatorname{span}_{\mathbb{C}}\left\{\psi, \psi^{\prime}, \psi^{\prime \prime}\right\}$, i.e., to $\hat{L}$ being self-dual in the sense that $\hat{L} \cong \hat{L}^{d}$ with respect to the isomorphism induced by $\Omega$. This shows that the twistor lift of a spherical or hyperbolic superminimal curve in $\mathbb{H}^{1}$ is self-dual.

\section{ACKNOWLEDGMENT}

We would like to thank Ulrich Pinkall for directing us to the subject and for supporting our project. We thank Iskander Taimanov, Franz Pedit and Ekkehard Tjaden for helpful discussions.

\section{REFERENCES}

[1] Uwe Abresch. Constant mean curvature tori in terms of elliptic functions. J. Reine Angew. Math. 374 (1987), 169-192. MR876223 (88e:53006)

[2] Alexander I. Bobenko. Surfaces of constant mean curvature and integrable equations. (Russian) Uspekhi Mat. Nauk 46 (1991), no. 4 (280), 3-42, 192; translation in Russian Math. Surveys 46 (1991), no. 4, 1-45. MR1138951 (93b:53009)

[3] Christoph Bohle, Constrained Willmore tori in the 4-sphere. J. Diff. Geom. 86 (2010), 71131.

[4] Christoph Bohle, Katrin Leschke, Franz Pedit, and Ulrich Pinkall, Conformal maps from a 2-torus to the 4-sphere. To appear in J. Reine Angew. Math.

[5] Christoph Bohle and G. Paul Peters. Bryant surfaces with smooth ends. Comm. Anal. Geom. 17 (2009), 587-619. MR2601345

[6] Robert L. Bryant. Conformal and minimal immersions of compact surfaces into the 4-sphere. J. Differential Geom. 17 (1982), no. 3, 455-473. MR679067 (84a:53062)

[7] Robert L. Bryant. A duality theorem for Willmore surfaces. J. Differential Geom. 20 (1984), no. 1, 23-53. MR772125 (86j:58029)

[8] Robert L. Bryant. Surfaces of mean curvature one in hyperbolic space. Théorie des variétés minimales et applications (Palaiseau, 1983-1984). Astérisque No. 154-155 (1987), 12, 321347, 353 (1988). MR955072 
[9] Robert L. Bryant. Surfaces in conformal geometry. The mathematical heritage of Hermann Weyl (Durham, NC, 1987), 227-240, Proc. Sympos. Pure Math., 48, Amer. Math. Soc., Providence, RI, 1988. MR974338 (89m:53102)

[10] Francis E. Burstall, Dirk Ferus, Katrin Leschke, Franz Pedit, and Ulrich Pinkall. Conformal Geometry of Surfaces in $S^{4}$ and Quaternions, Lecture Notes in Mathematics 1772, Springer, Berlin, 2002. MR1887131 (2004a:53058)

[11] Celso J. Costa. Example of a complete minimal immersion in $\mathbb{R}^{3}$ of genus one and three embedded ends. Bol. Soc. Brasil. Mat. 15 (1984), no. 1-2, 47-54. MR794728 (87c:53111)

[12] Norio Ejiri. Willmore surfaces with a duality in $S^{N}(1)$. Proc. London Math. Soc. (3) 57 (1988), no. 2, 383-416. MR950596 (89h:53117)

[13] Dirk Ferus, Katrin Leschke, Franz Pedit, and Ulrich Pinkall. Quaternionic holomorphic geometry: Plücker formula, Dirac eigenvalue estimates and energy estimates of harmonic 2-tori. Invent. Math. 146 (2001), 507-593. MR1869849 (2003a:53057)

[14] Thomas Friedrich. On surfaces in four-space. Ann. Global Anal. Geom. 2 (1984), no. 3, 257-287. MR777909 (86h:53061)

[15] Thomas Friedrich. The geometry of $t$-holomorphic surfaces in $S^{4}$. Math. Nachr. 137 (1988),49-62. MR968986 (90a:53075)

[16] Thomas Friedrich. On superminimal surfaces. Arch. Math. (Brno) 33 (1997), no. 1-2, 41-56. MR:1464300 (98h:53092)

[17] Philipp Griffith and Joseph Harris. Principles of algebraic geometry. Pure and applied mathematics, John Wiley \& Sons, New York, 1978, Wiley Classics Library Edition, 1994. MR.507725 (80b:14001)

[18] Robert D. Gulliver II, Robert Osserman, and Halsey L. Royden. A theory of branched immersions of surfaces. Amer. J. Math. 95 (1973), 750-812. MR0362153 (50:14595)

[19] Udo Hertrich-Jeromin, Emilio Musso, and Lorenzo Nicolodi, Möbius geometry of surfaces of constant mean curvature 1 in hyperbolic space. Ann. Global Anal. Geom. 19 (2001), no. 2, 185-205. MR1826401 (2002a:53079)

[20] Udo Hertrich-Jeromin. Introduction to Möbius Differential Geometry. Lecture Notes Series 300, Cambridge University Press, 2003. MR2004958 (2004g:53001)

[21] Nigel J. Hitchin. Harmonic maps from a 2-torus to the 3-sphere. J. Differential Geom. 31 (1990), no. 3, 627-710. MR:1053342 (91d:58050)

[22] David A. Hoffman and William H. Meeks III. A complete embedded minimal surface in $\mathbb{R}^{3}$ with genus one and three ends. J. Differential Geom. 21 (1985), no. 1, 109-127. MR806705 (87d:53008)

[23] Boris Konopelchenko. Induced surfaces and their integrable dynamics. Stud. Appl. Math. 96 (1996), no. 1, 9-51. MR.1365273 (96i:53011)

[24] William H. Meeks, Antonio Ros, and Herold Rosenberg. The global theory of minimal surfaces in flat spaces. Lectures given at the 2nd C.I.M.E. Session held in Martina Franca, July 7-14, 1999. Edited by Gian Pietro Pirola. Lecture Notes in Mathematics, 1775. Springer-Verlag, Berlin, 2002. MR1901611 (2003i:53012)

[25] Sebastián Montiel. Willmore two-spheres in the four-sphere. Trans. Amer. Math. Soc. 352 (2000), no. 10, 4469-4486. MR1695032 (2001b:53080)

[26] Emilio Musso. Willmore surfaces in the four-sphere. Ann. Global Anal. Geom. 8 (1990), no. 1, 21-41. MR 1075237 (92g:53059)

[27] Robert A. Osserman. A survey of minimal surfaces. Second edition. Dover Publications, Inc., New York, 1986. MR852409 (87j:53012)

[28] Franz Pedit and Ulrich Pinkall. Quaternionic analysis on Riemann surfaces and differential geometry. Doc. Math. J. DMV, Extra Volume ICM Berlin 1998, Vol. II, 389-400. MR.1648089 (2000c:53053)

[29] G. Paul Peters. Soliton Spheres. Thesis, TU-Berlin, urn:nbn:de:kobv:83-opus-8422 2004.

[30] Ulrich Pinkall and Ivan Sterling. On the classification of constant mean curvature tori. Ann. of Math. (2) 130 (1989), no. 2, 407-451. MR.1014929 (91b:53009)

[31] Jörg Richter. Conformal maps of a Riemann surface into the space of quaternions. Thesis, TU-Berlin, 1997.

[32] Iskander A. Taimanov. Modified Novikov-Veselov equation and differential geometry of surfaces. Solitons, geometry, and topology: on the crossroad, 133-151, Amer. Math. Soc. Transl. Ser. 2, 179, Amer. Math. Soc., Providence, RI, 1997. MR1437161 (98c:53071) 
[33] Iskander A. Taimanov. The Weierstrass representation of closed surfaces in $R^{3}$. (Russian) Funktsional. Anal. i Prilozhen. 32 (1998), no. 4, 49-62, 96; translation in Funct. Anal. Appl. 32 (1998), no. 4, 258-267. MR1678856(2000d:53006)

[34] Iskander A. Taimanov. The Weierstrass representation of spheres in $\mathbb{R}^{3}$, the Willmore numbers, and soliton spheres. Tr. Mat. Inst. Steklova, 225 (1999), Solitony Geom. Topol. na Perekrest., 339-361; translation in Proc. Steklov Inst. Math. (1999), no. 2 225, 322-343. MR:1725951 (2001g:37135)

[35] Iskander A. Taimanov. The two-dimensional Dirac operator and the theory of surfaces. (Russian) Uspekhi Mat. Nauk 61 (2006), no. 1(367), 85-164; translation in Russian Math. Surveys 61 (2006), no. 1, 79-159. MR2239773 (2007k:37098)

[36] Henry C. Wente. Counterexample to a conjecture of H. Hopf. Pacific J. Math. 121 (1986), no. 1, 193-243. MR815044 (87d:53013)

[37] Thomas J. Willmore, Riemannian geometry. Oxford University Press, Oxford, New York, 1993. MR.1261641 (95e:53002)

Mathematisches Institut, Universität TüBingen, Auf Der Morgenstelle 10, 72076 Tübingen, Germany

E-mail address: bohle@mathematik.uni-tuebingen.de

Institut für Mathematik, Technische Universität Berlin, Strasse des 17. Juni 136, 10623 Berlin, Germany

E-mail address: peters@math.tu-berlin.de 